\title{
The faunal drugstore: Animal-based remedies used in traditional medicines in Latin America
}

\author{
Rômulo RN Alves ${ }^{1 *}$, Humberto N Alves ${ }^{2}$
}

\begin{abstract}
Zootherapy is the treatment of human ailments with remedies made from animals and their products. Despite its prevalence in traditional medical practices worldwide, research on this phenomenon has often been neglected in comparison to medicinal plant research. This review discusses some related aspects of the use of animal-based remedies in Latin America, identifies those species used as folk remedies, and discusses the implications of zootherapy for public health and biological conservation. The review of literature revealed that at least 584 animal species, distributed in 13 taxonomic categories, have been used in traditional medicine in region. The number of medicinal species catalogued was quite expansive and demonstrates the importance of zootherapy as an alternative mode of therapy in Latin America. Nevertheless, this number is certainly underestimated since the number of studies on the theme are very limited. Animals provide the raw materials for remedies prescribed clinically and are also used in the form of amulets and charms in magic-religious rituals and ceremonies. Zootherapeutic resources were used to treat different diseases. The medicinal fauna is largely based on wild animals, including some endangered species. Besides being influenced by cultural aspects, the relations between humans and biodiversity in the form of zootherapeutic practices are conditioned by the social and economic relations between humans themselves. Further ethnopharmacological studies are necessary to increase our understanding of the links between traditional uses of faunistic resources and conservation biology, public health policies, sustainable management of natural resources and bio-prospecting.
\end{abstract}

\section{Introduction}

Throughout human history, people have used various materials from nature to cure their illnesses and improve their health [1]. Traditional human populations have a broad natural pharmacopoeia consisting of wild plant and animal species. According to the World Health Organization, 80 percent of the developing world's rural population depends on traditional medicines for its primary healthcare needs [2]. In many parts of the world, traditional medicine is the preferred form of health care, and remains the most available and affordable form of therapy in low income countries [3]. Ingredients sourced from wild plants and animals are not only used in traditional medicines, but are also increasingly valued as raw materials in the preparation of modern medicines and herbal preparations. Nature has been the source of medicinal agents for thousands of years, and an impressive number of modern

\footnotetext{
* Correspondence: romulo_nobrega@yahoo.com.br

'Departamento de Biologia, Universidade Estadual da Paraíba, Avenida das Baraúnas, Campina Grande, Paraíba 58109-753, Brasil

Full list of author information is available at the end of the article
}

drugs have been isolated from natural sources, many based on their use in traditional medicine [1].

The use of biological resources for various therapies has been documented in many different parts of the world [4-16]. Plants and animals have been used as medicinal sources since ancient times [1,2,17-22], and even today animal and plant-based pharmacopeias continue to play an essential role in world health care $[1,2,4]$. Although plants and plant-derived materials make up the majority of ingredients used in most traditional medical systems globally, whole animals, animal parts, and animal-derived products (e.g., urine, fat, etc.) also constitute important elements of the Materia Medica [2,23-25].

The use of organs or parts of animals as medicine is the basis of many traditional therapeutic practices [25]. Zootherapy is the treatment of human ailments with remedies made from animals and their products [2]. As Marques [26] states, "all human culture which presents a structured medical system will utilize animals as medicines". The phenomenon of zootherapy is marked both by a broad geographical distribution and very deep historical
C Biomed Central

(c) 2011 Alves and Alves; licensee BioMed Central Ltd. This is an Open Access article distributed under the terms of the Creative Commons Attribution License (http://creativecommons.org/licenses/by/2.0), which permits unrestricted use, distribution, and reproduction in any medium, provided the original work is properly cited. 
origins. In modern societies, zootherapy constitutes an important alternative among many other known therapies practiced worldwide [2,27-39]. Despite its prevalence in traditional medical practices worldwide, research on this phenomenon has often been neglected in comparison to medicinal plant research [2]. Traditionally, medicinal animals have received little attention from ethnobiologists and anthropologists. It is only within the past few decades that researchers have begun to systematically investigate the uses of medicinal plants, and an awareness of the variety and importance of nonbotanical remedies (of animal and mineral origin) is emerging [40]. In spite of the recent surge in publications about zootherapeutics the subject is still far from being well covered, and even more distant from being exhausted. The lack of zootherapeutic studies in Latin America (and in the world in general) has contributed to an underestimation of the importance of zootherapeutic resources $[41,42]$.

Many cultures still employ traditional medicine that includes animal-derived remedies. Probably the most famous of these are the Chinese, who use animals for a variety of ailments. Lesser known and studied, though just as varied and rich is Latin America's long tradition of animal-remedies for all kinds of ailments. Latin America's rich biological and cultural diversity makes it an exceptional location in which to examine and increase our knowledge of faunistic resources used as in traditional folk medicine, to draw attention to their importance in public health, and protect traditional knowledge and biodiversity.

Latin America is outstanding both because of its great wealth of genetic resources and complex cultural diversity [43-46]. The adaptation of the various human groups to the region's rich biological resources generated invaluable local knowledge systems that include extensive information on plant and animal uses in general [43,47-59]. In that context, the aim of this study was to provide an overview of the use of medicinal animals in Latin America, identify those species used as folk remedies, and discuss the implications of zootherapy for public health and biological conservation.

\section{Methods}

\section{Study area}

Latin America is a vast region spanning parts of North America, almost all of South America, and much of the West Indies. It encompasses 19 countries as well as Puerto Rico, a commonwealth territory of the United States, and, arguably, even parts of southwestern United States [46]. In Latin America and the Caribbean, the population stood at 577 million in 2008 and is projected to increase to 778 million by 2050 [60]. It is the most urbanized region in the developing world, with around three-quarters of the population living in urban areas [43]. The population of Latin America is a composite of ancestries, ethnic groups, and races, making the region one of the most-if not the most-racially and ethnically diverse in the world. The specific composition varies from country to country: Some countries have a predominance of a mixed population, in others people of Amerindian origin are a majority, some are dominated by inhabitants of European ancestry, while others are primarily of African descent. Most or all Latin American countries also have large Asian minorities. Europeans are the largest single group, and they and people of part-European ancestry combine to make up approximately $80 \%$ of the population of the subcontinent [61].

Latin America is one of the world's principal culture regions. It is distinguished from other world regions by a set of common cultural traits that include language, religion, social values, and civic institutions deriving principally from the Iberian Peninsula. Spanish and Portuguese are predominant languages. Catholicism is practiced by a vast majority of the region's inhabitants, and social customs and civic institutions bear many similarities to those in Spain. Nevertheless, the region is not culturally monolithic. Indigenous cultures and peoples have influenced national and subnational cultures within region, affecting language, religion, music, food habits, social customs, and civic institutions. The descendents of African slaves have also influenced the region's culture, although their effects have been most pronounced in Brazil, the Caribbean, and coastal areas of Central and northern South America. The cultural impact of other immigrants, including those from Italy, Asia, the Middle East, and even a few from North America has been minor [46].

\section{Procedures}

In order to examine the diversity of animals used in traditional medicine in Latin America, all available references or reports of folk remedies based on animal sources were examined [4,7-11,29-31,36,38,40,42, 62-193]. Information was gathered from published articles, books and book chapters, theses and dissertations, undergraduate theses, as well as from reports, and abstracts available at international online databases such as Web of Science, Scopus and Google Scholar and journals' web sites. The resulting database encompassed information on species, family names, and conditions to which remedies are prescribed. Only taxa that could be identified to species level were included in the database. Scientific names provided in publications were updated according to the ITIS Catalogue of Life: 2011 Annual Checklist [194].

\section{Medicinal fauna of Latin America}

The use of medicinal fauna in Latin America has been the focus of some ethnozoological research over the last two decades, mainly in countries such as Brazil, Mexico 
and Bolívia. These studies have demonstrated the importance of zootherapy to both urban and rural populations. This is not surprising, considering the rich biological resources and cultural of the region, that generated invaluable local knowledge systems that include extensive information on animal uses in general and medicinally useful species, in particular.

A review of the literature revealed that at least 584 animal species have been used in traditional medicine in Latin America (Table 1). The high number of animals used as medicine is not surprising given the important role played by wildlife as a source of medicines in different parts of the world. Nevertheless, the number is certainly underestimated since the amount of studies on the theme are very limited. It is self-evident that there is an urgent need for more studies into zootherapeutic practices in the region.

The species catalogued comprised 13 taxonomic categories, belonging to 215 families. The groups with the largest numbers of medicinal species were: mammals (with 130 species), followed by birds (122), fishes (110), reptiles (95) and insects (54) (Figure 1). Most medicinal animals recorded are vertebrates. Species of this group are also used frequently at countries of Europe, Africa and Asia countries [16,27,33,34,37,39,195-197]. Examples of animals used as medicine in Latin America is shown in Figure 2.

Most animals used as medicine were native to the Latin America, with the exception of Hippocampus spinosissimus, $H$. trimaculatus and Panthera leo and domestic exotic species (eg. Bos taurus, Capra hircus and Ovis aries). This finding demonstrates the importance of local biodiversity in furnishing folk medicines, in agreement with previous studies $[7,8,42]$ which observed that faunal composition, accessibility, and availability directly influence the types of zootherapeutic items used in any given region. The use of the local fauna generally reduces the acquisition costs of commercial agents, and our results are in agreement with Apaza et al. [32], who noted a reduction in the cost of acquiring animal products in regions with abundant faunal resources. The medicinal use of wild exotic animals in Latin America indicates existence of international trade routes for medicinal species, a situation previously reported by Convention on International Trade in Endangered Species of Wild Fauna and Flora - CITES [198]. One excellent example are the seahorses. According to Baum and Vincent [79], the largest market for dried seahorses in Central America was for use as Traditional Chinese Medicine. These authors reported that three retailers in Panama City's Chinatown imported an estimated total of 18-27 kg dried seahorses annually from Hong Kong. These included $H$. spinosissimus and
H. trimaculatus, which are found in the Red Sea and Indian Ocean, South-east Asia and Australia.

Some widespread species are used in different countries, such as Tupinambis spp. and Boa constrictor (in Argentina and Brazil), Tapirus terrestris (in Brazil and Bolivia) and Caudisona durissa in Mexico and Brazil $[8,32,38,189]$. Despite the fact that technical studies recording the use of animals in traditional medicine are all relatively recent, an analysis of historical documents and archaeological research indicated that animals have been used in traditional medicines since ancient times in Latin America [36,99,152-154,199]. In Brazil, for example, animal species have been used medicinally by indigenous societies for millennia. During his voyage through the interior of Brazil in the nineteenth century, Von Martius [200] recorded many natural medicines used by the Amerindian tribes he encountered, such as fresh caiman fat applied to alleviate rheumatism [115]. Even today, caiman fat (Cayman latirostris, Melanosuchus niger and Paleosuchus palpebrosus) is used in rural and urban communities to treat rheumatism [8]. Some examples of animals that have been used in Brazil since colonial times include: Iguana iguana (Iguanidae), Caiman latirostris, Crotalus durissus, and Micrurus ibiboboca. Similarly, a historical revision of the therapeutic uses of animals as described in Colonial chronicles from Mexico [99] revealed a total of 27 birds used as medicinal resource, showing their importance on this issue for the Ancient Mexican people.

\section{Illnesses and remedies}

Animal-derived remedies were used for treating various diseases (See Table 1). A single illness could be treated by various animal species (e.g., 215 animal species were used in the treatment of asthma and 95 in the treatment of rheumatism), and many species were prescribed for treating multiple illnesses, as in the case of the products obtained from the teju (Tupinambis teguixin) and the snake boa (Boa constrictor), which were indicated to treat 29 and 30 conditions, respectively. The reported multiple therapeutic actions and the use of various animals for the same condition presumes different properties either of parts used or of modes of preparation, corroborating the remark by Iwu [201] that different chemical constituents are enhanced by different factors, such as preparation, dosage, or part used. Additionally, the possibility of using various remedies for the same ailment is popular because it permits adapting to the availability of the animals [7]. The fact that some medicinal animals are being used for the same purpose suggest that different species can share similar medicinal properties and might indicate the pharmacological effectiveness of these zootherapeutic remedies. 
Table 1 Medicinal animals and its respective uses in popular medicine in Latin America

\begin{tabular}{|c|c|c|}
\hline Family/Species & $\begin{array}{l}\text { Conditions to which } \\
\text { remedies are prescribed }\end{array}$ & References \\
\hline \multicolumn{3}{|l|}{ PORIFERA } \\
\hline \multicolumn{3}{|l|}{ Spongiidae } \\
\hline Spongia officinalis Linnaeus, 1759 & Unspecified & [124] \\
\hline \multicolumn{3}{|l|}{ CNIDARIANS } \\
\hline \multicolumn{3}{|l|}{ Mussidae } \\
\hline Mussismilia harttii (Verril, 1868) & $\begin{array}{l}\text { Vaginal discharge, } \\
\text { diarrhoea }\end{array}$ & {$[11,149]$} \\
\hline \multicolumn{3}{|l|}{ Physaliidae } \\
\hline $\begin{array}{l}\text { Physalia physalia (Linnaeus, 1758) - Portuguese-man-of-war, } \\
\text { jellyfish }\end{array}$ & Asthma & {$[7-9,11,31,146]$} \\
\hline \multicolumn{3}{|l|}{ MOLLUSCS } \\
\hline \multicolumn{3}{|l|}{ Ampullariidae } \\
\hline Pomacea lineata (Spix, 1827) - Snail, Clam & Asthma, sprains, boils, ulcer & {$[7-9,11,31,97,115,146,161,169]$} \\
\hline \multicolumn{3}{|l|}{ Megalobulimidae } \\
\hline Megalobulimus oblongus (Mueller, 1774) -clam & Asthma & {$[11,66]$} \\
\hline \multicolumn{3}{|l|}{ Donacidae } \\
\hline Iphigenia brasiliana (Lamarck, 1818) - giant coquina & Teething & {$[80,146]$} \\
\hline \multicolumn{3}{|l|}{ Loliginidae } \\
\hline Loligo vulgaris Lamarck, 1798 & Unspecified & {$[80]$} \\
\hline \multicolumn{3}{|l|}{ Cassidae } \\
\hline Cassis tuberosa (Linnaeus, 1758) - Conch & Asthma & {$[99,146]$} \\
\hline \multicolumn{3}{|l|}{ Littorinidae } \\
\hline Littorina angulifera (Lamarck, 1822) - Periwinkle snail & $\begin{array}{l}\text { Chesty cough, shortness of } \\
\text { breath }\end{array}$ & {$[7-9,146]$} \\
\hline \multicolumn{3}{|l|}{ Lucinidae } \\
\hline Phacoides pectinatus (Gmelin, 1791) - Shellfish & Sexual impotence & {$[11,146]$} \\
\hline \multicolumn{3}{|l|}{ Melongenidae } \\
\hline Pugilina morio (Linnaeus, 1758) - Conch & Sexual impotence & {$[11,99,146]$} \\
\hline \multicolumn{3}{|l|}{ Mytilidae } \\
\hline Mytella charruana (Orbigny, 1842) - Mussel, Shellfish & $\begin{array}{l}\text { Ophthalmological } \\
\text { problems }\end{array}$ & {$[11,99,146]$} \\
\hline Mytella guyanensis Lamarck (1819) - Mussel, Shellfish & Weakness & {$[7-9,146]$} \\
\hline \multicolumn{3}{|l|}{ Ostreidae } \\
\hline Crassostrea rhizophorae (Guilding, 1828) Mangrove oyster & $\begin{array}{l}\text { Osteoporosis, pneumonia, } \\
\text { stomach ache, cancer, flu, } \\
\text { weakness, pain relief in } \\
\text { injuries caused by the } \\
\text { dorsal fin spine of a } \\
\text { species of catfish, anaemia, } \\
\text { tuberculosis }\end{array}$ & {$[7-9,146]$} \\
\hline \multicolumn{3}{|l|}{ Strombidae } \\
\hline Aliger pugilis Linnaeus, 1758 - West Indian fighting conch & Sexual impotence & {$[11,99,146]$} \\
\hline \multicolumn{3}{|l|}{ Teredinidae } \\
\hline Neoteredo reynei (Bartsch, 1920) - Shipworm & Anaemia, tuberculosis & {$[69,146]$} \\
\hline Teredo pedicellata Quatrefages, 1849 & Tuberculosis & {$[99,146]$} \\
\hline \multicolumn{3}{|l|}{ Vasidae } \\
\hline Turbinella laevigata (Anton, 1839) - Conch & Sexual impotence & {$[99,146]$} \\
\hline \multicolumn{3}{|l|}{ Veneridae } \\
\hline Anomalocardia brasiliana (Gmelin, 1791) - Clam, shellfish & Asthma, flu, stomach ache & {$[7-9,146]$} \\
\hline
\end{tabular}


Table 1 Medicinal animals and its respective uses in popular medicine in Latin America (Continued)

\begin{tabular}{|c|c|c|}
\hline Octopodidae & & \\
\hline Octopus vulgaris (Cuvier, 1799) - common octopus & Unspecified & [117] \\
\hline ANNELIDA & & \\
\hline Lumbricidae & & \\
\hline Lumbricus terrestris (Linnaeus, 1758) & Inflamatory process & [87] \\
\hline CHELICERATA & & \\
\hline Bothriuridae & & \\
\hline Bothriurus asper Pocock, 1893 - black scorpion & Ethnoveterinary use & {$[127,178]$} \\
\hline Buthidae & & \\
\hline Rhopalurus rochai (Borelli 1910) & $\begin{array}{l}\text { Scorpion bite, } \\
\text { ethnoveterinary use }\end{array}$ & {$[127,151,178]$} \\
\hline CRUSTACEANS & & \\
\hline Calappidae & & \\
\hline Calappa ocellata Holthuis, 1958 - Ocellate box crab & Asthma, osteoporosis & {$[11,36,146]$} \\
\hline Gecarcinidae & & \\
\hline Cardisoma guanhumi Latreille, 1825 - Blue land crab & $\begin{array}{l}\text { Asthma, bronchitis, } \\
\text { wounds, boils }\end{array}$ & {$[11,146]$} \\
\hline Grapsidae & & \\
\hline Goniopsis cruentata (Latreille, 1802) - Mangrove root crab & Epilepsy, venereal disease & {$[7-9,11,99]$} \\
\hline Plagusia depressa (Fabricius, 1775) - Tidal spray crab & Epilepsy & {$[11,99,146]$} \\
\hline Hippidae & & \\
\hline Emerita portoricensis Schmitt, 1935 - Puerto Rican sand crab & Earache & {$[11,99,146]$} \\
\hline Ocypodidae & & \\
\hline Ocypode quadrata (JC Fabricius, 1787) - Ghost crab & $\begin{array}{l}\text { Asthma, haemorrhage in } \\
\text { women, flu, to alleviate the } \\
\text { symptoms of intoxication } \\
\text { with poison of niquim } \\
\text { (Pisces, Batrachoididae) }\end{array}$ & {$[7-9,119,146]$} \\
\hline Ucides cordatus (Linnaeus, 1763) - Swamp Land crab & $\begin{array}{l}\text { Haemorrhage in women, } \\
\text { incontinence urinary, } \\
\text { osteoporosis, cough, } \\
\text { asthma, tuberculosis, } \\
\text { womb disorders, arthrosis, } \\
\text { bronchitis }\end{array}$ & {$[7-9,119,146]$} \\
\hline Uca maracoani (Latreille, 1802) - Fiddler crab & Asthma, whooping cough & {$[7-9,119,146]$} \\
\hline Palaemonidae & & \\
\hline $\begin{array}{l}\text { Macrobrachium carcinus (Linnaeus, 1758) - Bigclaw river shrimp, } \\
\text { Painted river prawn }\end{array}$ & Amnesia & {$[11,99,146]$} \\
\hline $\begin{array}{l}\text { Macrobrachium acanthurus (Wiegmann, 1836) - Cinnamon river } \\
\text { shrimp }\end{array}$ & $\begin{array}{l}\text { Irritation when milk teeth } \\
\text { are erupting }\end{array}$ & {$[7-9,146]$} \\
\hline Macrobrachium borellii (Nobili, 1896) - Freshwater shrimp & $\begin{array}{l}\text { Irritation when milk teeth } \\
\text { are erupting }\end{array}$ & {$[7-9,146]$} \\
\hline Penaeidae & & \\
\hline Xiphopenaeus schmitti (Burkenroad, 1936) - Southern white shrimp & $\begin{array}{l}\text { Irritation when milk teeth } \\
\text { are erupting, skin spots }\end{array}$ & {$[7-9,146]$} \\
\hline Xiphopenaeus kroyeri (Heller, 1862) - Atlantic seabob & $\begin{array}{l}\text { Irritation when milk teeth } \\
\text { are erupting, skin spots }\end{array}$ & {$[7-9,146]$} \\
\hline Pseudosquillidae & & \\
\hline Cloridopsis dubia (H. M. Edwards, 1837) - Mantis shrimp & Asthma & {$[7-9,146,164]$} \\
\hline Armadillidiidae & & \\
\hline Armadillidium vulgare (Latreille, 1804) - Pillbug & Asthma & [177] \\
\hline
\end{tabular}


Table 1 Medicinal animals and its respective uses in popular medicine in Latin America (Continued)

Aratus pisoni (H. Milne Edwards, 1837) - Mangrove crab
Epilepsy, to alleviate the symptoms of intoxication with poison of Colomesus psittacus

\section{Mithracidae}

Mithrax hispidus (J. F. W. Herbst, 1790) - coral clinging crab Burns [151]

\section{Portunidae}

Callinectes bocourti A. Milne-Edwards, 1879 - Bocourt swimming Unspecified crab

Callinectes exasperatus Gerstaecker, 1856 - rugose swimming crab Unspecified [80]

\section{INSECTS}

\section{Apidae}

Apis mellifera (Linnaeus, 1758) - Africanised honey bee

Cough, flu, rheumatism,

tuberculosis, bronchits,

$[7,9,11,99,146]$

hoarseness, ulcer, diabetes,

verminosis, headache, giddiness, backache, wounds, burns, mumps, varicose veins, arthrosis, cellulitis, amoebiasis, sore throat, asthma, anaemia catarrh

\begin{tabular}{|c|c|c|}
\hline Cephalotrigona capitata (Smith, 1854) - Bee & Snake bite & {$[11,146]$} \\
\hline Frieseomelitta silvestrii (Friese, 1902) - Stingless bee & Flu & {$[11,146]$} \\
\hline Frieseomelitta varia (Lepeletier, 1836) - Bee & Gonorrhea & [136] \\
\hline Melipona compressipes (Fabricius, 1804) - Stingless bee & Asthma, cough & {$[7-9,146]$} \\
\hline Melipona mandacaia Smith, 1863 - Stingless bee & Wounds & {$[146,170]$} \\
\hline $\begin{array}{l}\text { Melipona quadrifasciata Lepeletier, } 1836 \text { - Neotropical stingless } \\
\text { bee }\end{array}$ & Snake bite & {$[146,187]$} \\
\hline
\end{tabular}

Melipona scutellaris (Latreille, 1811) - Stingless bee

Headache, migraine, stroke, $[7,9,98,109,110,164]$ verminosis, stomach ache, tuberculosis, haemorrhage, cataracts, mycosis in the mouth, flu, cancer, asthma, bronchits, intestinal disorders, cough, sexual impotence, ophthalmological problems, weakness, thrombosis, amoebiasis, snake bite, rabies, sinusitis, fatigue

\begin{tabular}{|c|c|c|}
\hline Melipona subnitida (Ducke, 1910) - Honey bee & Flu, sore throat & {$[7-9,11,109,110,118]$} \\
\hline Melipona fulva (Lepeletier, 1836) - bee & Unspecified & [86] \\
\hline Melipona asilvai Moure, 1971 - bee & Headache, flu & {$[121]$} \\
\hline Melipona marginata Lepeletier, 1836 - bee & Cough & [81] \\
\hline Partamona Cupira (Smith, 1863) - Stingless bee & $\begin{array}{l}\text { Sore throat, swelling, } \\
\text { headache, thrombosis, } \\
\text { stroke, leucoma, "slightly } \\
\text { clean", cuts, wounds, } \\
\text { cough, catarrh, toaday, } \\
\text { sinusitis, effusion }\end{array}$ & {$[11,99,109,110,128,164,165,187]$} \\
\hline Partamona seridoensis Pedro \& Camargo, 2003 - Cupira bee & Ethnoveterinary uses & {$[127,178]$} \\
\hline Plebeia cf. emerina Friese, 1900 - Mosquito & Mycosis in the mouth area & {$[11,118,166,170,177]$} \\
\hline Tetragonisca angustula Latreille, 1811 - Bee & $\begin{array}{l}\text { Cataracts, sinusitis, cough, } \\
\text { flu, ophthalmological } \\
\text { problems, sore throat, } \\
\text { leucoma }\end{array}$ & {$[7-9,71,112,170]$} \\
\hline
\end{tabular}


Table 1 Medicinal animals and its respective uses in popular medicine in Latin America (Continued)

Trigona mosquito Lutz, 1931 - Stingless bee

Trigona spinipes (Fabricius, 1793) - Stingless bee

Lestrimelitta limao (Smith, 1863) - bee

\section{Forficulidae}

Forficula auricularia Linnaeus, 1758

Tenebrioninae

Eleodes spinipes (Solier, 1848)

\section{Cerambycidae}

Macrodontia cervicornis (Linné, 1758)

\section{Blattidae}

Periplaneta americana (Linnaeus, 1758) - American cockroach

To keep away from bad [103]

spirits

Unspecified

Heartburn, asthma,

\section{Cough}

Asthma, cough, flu, bronchits, acne, diabetes,

stroke, thrombosis,

migraine, itching, sore

throat, giddiness, weakness,

scabies, nasal congestion,

to induce abortion,

whooping cough, irritation

when milk teeth are

erupting, earache, epilepsy,

shortness of breath, late

menstruation, fatigue,

effusion

\section{Dizziness}

Earache, whooping cough

[81]

$[11,71,113]$

$[11,97,110,115,118,161,164,170]$

[103]

[82] stomach ache, intestinal

$[7,9,11,31,71,109,112,115,128,130,170]$

colic, earache, alcoholism,

epilepsy, vomit, boil,

haemorrhage, bronchits,

diarrhoea, gonorrhea,

panaris, cancer, stroke,

burns, menstrual cramps,

wounds, to suck a splinter

out of skin or flesh,

detoxification, avoiding

pregnancy

\section{Eurycotis manni (Rehn, 1916)}

Unspecified

[170]

\section{Blaberidae}

Rhyparobia maderae (Fabricius, 1781) - Madeira cockroach

Asthma

[50]

\section{Chrysomelidae}

Coraliomela brunnea Thumberg, 1821 - Fake cockroach

Epilepsy

$[8,11,132,146]$

Pachymerus cf. nucleorum (Fabricius, 1792) - Caterpillar

Earache, stroke, swelling,

$[71,112,159,170]$

wounds, seborrheic

dermatitis, inflammation,

thrombosis

\begin{tabular}{|c|c|c|}
\hline \multicolumn{3}{|l|}{ Curculionidae } \\
\hline Rhynchophorus palmarum Linnaeus, 1758 - Pest of coconut palm & Fever, headache, boils & {$[149,153]$} \\
\hline Rhinostomus barbirostris Fabricius, 1775 - Pest of coconut palm & Fever, headache, boils & {$[149,153]$} \\
\hline Rhina barbirostris Champion, G.C., 1910 & Fever, headache, boils & [153] \\
\hline \multicolumn{3}{|l|}{ Formicidae } \\
\hline Atta cephalotes (Linnaeus, 1758) - Leaf-cutter ant & Sore throat & {$[7-9,11,66,144]$} \\
\hline Atta serdens (Linnaeus, 1758) - Leaf-cutting & $\begin{array}{l}\text { Stomach ache, heart } \\
\text { diseases, chest palpations }\end{array}$ & {$[11,70,71,113]$} \\
\hline Dinoponera quadriceps (Santschi, 1921) - Bullet ant & Asthma & {$[7-9,11,109,170]$} \\
\hline Acromyrmex landolti (Emery, 1980)- ant & Asthma & [66] \\
\hline Solenopsis saevissima (Smith, 1855) - Ant & Wart & [102] \\
\hline
\end{tabular}


Table 1 Medicinal animals and its respective uses in popular medicine in Latin America (Continued)

\section{Gryllidae}

Acheta domesticus (Linnaeus, 1758) - House cricket

Scabies, asthma, eczema,
lithiasis, earache, oliguresis,

$[11,113]$

rheumatism, urine

retention, children that

urinate in bed and speak

with lateness, incontinence

urinary, ophthalmological

problems

\begin{tabular}{|c|c|c|}
\hline Paragryllus temulentus Saussure 1878 - Cricket & Rheumatism & [69] \\
\hline Gryllus assimilis (Fabricius, 1775) - cricket & Warts & [81] \\
\hline \multicolumn{3}{|l|}{ Meloidae } \\
\hline Palembus dermestoides (Fairmaire, 1893) - Peanut beeatle & $\begin{array}{l}\text { Sexual impotence, } \\
\text { ophthalmological } \\
\text { problems, rheumatism, } \\
\text { weakness }\end{array}$ & {$[11,71,113,163]$} \\
\hline Pseudomeloe andensis (Guérin Méneville 1992) & Warts & [189] \\
\hline \multicolumn{3}{|l|}{ Muscidae } \\
\hline Musca domestica (Linnaeus, 1758) - House fly & $\begin{array}{l}\text { Boil, baldness, eyesore, } \\
\text { external sebaceus lamps, } \\
\text { stye, spots in the face, } \\
\text { ophthalmological } \\
\text { problems, dermatosis, } \\
\text { cysties, erysipelas }\end{array}$ & {$[11,115,165]$} \\
\hline \multicolumn{3}{|l|}{ Pediculidae } \\
\hline Pediculus humanus Linnaeus, 1758 - Body louse, Head louse & Tootache & [159] \\
\hline \multicolumn{3}{|l|}{ Psychidae } \\
\hline Eurycotis manni Rehn, 1916 - Beetle & Headache & {$[11,71,115,128,146,177]$} \\
\hline Oiketicus kirbyi Guilding, 1827 - Case moth & $\begin{array}{l}\text { Asthma, earache, } \\
\text { haemorrhage }\end{array}$ & {$[11,113]$} \\
\hline \multicolumn{3}{|l|}{ Termitidae } \\
\hline Microcerotermes exignus (Hagen, 1858), Termite & $\begin{array}{l}\text { Asthma, bronchits, flu, } \\
\text { whopping cough }\end{array}$ & {$[11,70]$} \\
\hline Nasutitermes macrocephalus (Silvestri, 1903) - Termite & $\begin{array}{l}\text { Asthma, catarrh, bronchitis, } \\
\text { 'catarrh in the chest' } \\
\text { coughs, influenza, sore } \\
\text { throat, sinusitis, tonsillitis } \\
\text { and hoarseness }\end{array}$ & {$[128,130,144]$} \\
\hline Nasutitermes corninger (Motschulsky, 1855) - termite & Unspecified & [172] \\
\hline \multicolumn{3}{|l|}{ Vespidae } \\
\hline Apoica pallens (Oliv. 1791) - Paper wasp & $\begin{array}{l}\text { Thrombosis, ashtma, } \\
\text { giddiness, nasal } \\
\text { haemorrhage, } \\
\text { haemorrhage, stroke, } \\
\text { disorders after parturition, } \\
\text { ophthalmological } \\
\text { problems, mumps, late } \\
\text { menstruation }\end{array}$ & {$[11,71,112,118,146,166,170]$} \\
\hline Brachygastra lecheguana (Latreille, 1824) - Dark paper wasp & Cough, asthma & {$[11,113]$} \\
\hline Polistes canadensis (Linnaeus, 1758) - Wasp & Cough, whooping cough & {$[11,116,165]$} \\
\hline Polybia sericea (Olivier, 1791) - Wasp & Thrombosis & {$[11,118]$} \\
\hline Protopolybia exigua (Saussure, 1854) - Wasp & $\begin{array}{l}\text { Evil eye, tobaccoism, } \\
\text { ophthalmological problems }\end{array}$ & {$[11,118]$} \\
\hline Synoeca surinama (Linnaeus, 1767) - Paper wasp & $\begin{array}{l}\text { Asthma, shortness of } \\
\text { breath }\end{array}$ & {$[11,71,118]$} \\
\hline Protonectarina sylveirae (Saussure, 1854)- & $\begin{array}{l}\text { Mumps, hemorrhage, } \\
\text { blooding and menstrual } \\
\text { problems }\end{array}$ & [66] \\
\hline
\end{tabular}


Table 1 Medicinal animals and its respective uses in popular medicine in Latin America (Continued)

\section{ECHINODERMS}

\section{Echinasteridae}

\begin{tabular}{lll}
\hline Echinaster brasiliensis Müller \& Troschel, 1842 - Starfish & Asthma & {$[11,36,63,130]$} \\
\hline Echinaster echinophorus Lamarck, 1816 - Starfish & Asthma & {$[71,99,130,163]$} \\
\hline Echinometridae & & \\
\hline Echinometra lucunter (Linnaeus, 1758) - Rock boring urchin & Asthma & {$[11,36,99,114,119,130,146,163,228]$} \\
\hline Luidiidae & & {$[7-9,11,36,99,114,119,130,146,163,228]$} \\
\hline Luidia senegalensis Lamarck, 1916 - Starfish & $\begin{array}{l}\text { Asthma, cough, } \\
\text { metrorrhagia }\end{array}$ & \\
\hline Mellitidae & & {$[99]$} \\
\hline Mellita sexiesperforata (Leske, 1778) - Six holed keyhole urchin & Asthma, cough & {$[11,99,130]$} \\
\hline Mellita quinquiesperforata (Leske, 1778) & Asthma & \\
\hline Oreasteridae & & {$[7-9,30,31,66,109,128,146]$} \\
\hline Oreaster reticulatus (Linnaeus, 1758) - Starfish & Asthma & {$[68,146]$} \\
\hline Toxopneustidae & & Snake bite \\
\hline Lytechinus variegatus (Lamarck, 1816) - Green sea urchin &
\end{tabular}

\section{FISHES}

\section{Auchenipteridae}

Trachelyopterus galeatus (Linnaeus, 1766) - Driftwood Cat

Umbilical hernia, asthma, $\quad[71,97,161,163,228]$ sexual impotence

\begin{tabular}{|c|c|c|}
\hline Anostomidae & & \\
\hline Leporinus friderici (Bloch, 1794) - Frederici's leporinus & Earache & [188] \\
\hline Leporinus piau Fowler, 1941 Black piau & Rheumatism & [71] \\
\hline Leporinus steindachneri Eigenmann, 1907 & Problems with cholesterol & [109] \\
\hline Schizodon knerii (Steindachner, 1875) - White piau & Leucoma, edema & [97] \\
\hline \multicolumn{3}{|l|}{ Ariidae } \\
\hline Bagre bagre (Linnaeus, 1766) - Coco sea catfish & $\begin{array}{l}\text { pain relief in injuries } \\
\text { caused by the dorsal fin } \\
\text { spine of a species of } \\
\text { catfish }\end{array}$ & {$[114,115,163]$} \\
\hline Genidens barbus (Lacepède, 1803) - White sea catfish & $\begin{array}{l}\text { pain relief in injuries } \\
\text { caused by the dorsal fin } \\
\text { spine of a species of } \\
\text { catfish }\end{array}$ & {$[8,114,162]$} \\
\hline Genidens genidens (Cuvier, 1829) - catfish & $\begin{array}{l}\text { pain relief in injuries } \\
\text { caused by the dorsal fin } \\
\text { spine of a species of } \\
\text { catfish }\end{array}$ & {$[8,126]$} \\
\hline Sciadeichthys luniscutis (Valenciennes, 1837) - Catfish & $\begin{array}{l}\text { pain relief in injuries } \\
\text { caused by the dorsal fin } \\
\text { spine of a species of } \\
\text { catfish }\end{array}$ & {$[8,114,162]$} \\
\hline Genidens barbus (Lacepède, 1803 - catfish & Unspecified & [111] \\
\hline Cathorops spixii (Agassiz, 1829) - catfish & Unspecified & [80] \\
\hline \multicolumn{3}{|l|}{ Aspredinidae } \\
\hline Aspredo aspredo (Linnaeus, 1758) - Banjo, catfish & Asthma & {$[7-9]$} \\
\hline Aspredinichthys tibicen (Valenciennes, 1840) - Tenbarbed banjo & Asthma & [7-9] \\
\hline \multicolumn{3}{|l|}{ Balistidae } \\
\hline Balistes capriscus Gronow, 1854 - Grey triggerfish & Bronchits & [85] \\
\hline Balistes vetula (Linnaeus, 1758) - Queen triggerfish & $\begin{array}{l}\text { Stroke, asthma, thrombosis, } \\
\text { earache, Injuries caused by } \\
\text { fish species, haemorrhage, } \\
\text { ascites, schistosomiasis, } \\
\text { appendicitis, menstrual } \\
\text { cramps, gastritis }\end{array}$ & {$[7-9,114,162,228]$} \\
\hline
\end{tabular}


Table 1 Medicinal animals and its respective uses in popular medicine in Latin America (Continued)

\begin{tabular}{|c|c|c|}
\hline Batrachoididae & & \\
\hline Thalassophryne nattereri (Steindachner, 1876) - Venomous toadfish & $\begin{array}{l}\text { Injuries caused by fish } \\
\text { species }\end{array}$ & {$[4,7-9,114,115,162]$} \\
\hline Callichthyidae & & \\
\hline Callichthys callichthys (Linnaeus, 1758) - Armoured catfish & Asthma, umbilical hérnia & {$[4,8,97,114,115]$} \\
\hline Carcharhinidae & & \\
\hline Carcharhinus limbatus (Müller \& Henle, 1839) - Blackfin shark & Osteoporosis & [7-9] \\
\hline Carcharhinus porosus (Ranzani, 1840) - Smalltail shark & $\begin{array}{l}\text { Asthma, rheumatism, } \\
\text { wounds, inflammations, } \\
\text { osteoporosis, anaemia }\end{array}$ & {$[7-9,69,114]$} \\
\hline Carcharhinus leucas Nalenciennes, 1839) - shark & Unspecified & [30] \\
\hline Galeocerdo cuvier (Péron \& Lesueur, 1822) - Tiger shark & Osteoporosis & {$[7-9]$} \\
\hline $\begin{array}{l}\text { Rhizoprionodon lalandii (Müller \& Henle, 1839) - Brazilian sharpnose } \\
\text { shark }\end{array}$ & Rheumatism & {$[7-9]$} \\
\hline Rhizoprionodon porosus (Poey, 1861) - Sharpnose shark & Rheumatism & [7-9] \\
\hline Sphyrna lewini (Griffith \& Smith, 1834) - Scalloped hammerhead & $\begin{array}{l}\text { Asthma, wounds, } \\
\text { rheumatism, inflammation }\end{array}$ & {$[8,9,114,162,228]$} \\
\hline Salmonidae & & \\
\hline Oncorhynchus mykiss (Walbaum, 1792) - redband trout & $\begin{array}{l}\text { Rheumatism, bad smell of } \\
\text { feet }\end{array}$ & [148] \\
\hline Centropomidae & & \\
\hline Centropomus parallelus Poey, 1860 - Smallscale fat snook & Nephritis & [149] \\
\hline Centropomus undecimalis (Bloch, 1792) - Common snook & Edema in the legs & {$[7-9,114,162,228]$} \\
\hline Characidae & & \\
\hline Astyanax bimaculatus (Linnaeus, 1758) - Twospot astyanax & $\begin{array}{l}\text { Alcoholism, leishmaniosis, } \\
\text { skin burns, wounds, } \\
\text { rheumatism }\end{array}$ & {$[7-9,114,157,162,228]$} \\
\hline Paracheirodon axelrodi (Schultz, 1956) - Cardinal tetra & Ashtma & [82] \\
\hline Chalceus macrolepidotus Cuvier, 1818 & Unspecified & [82] \\
\hline Brycon nattereri Günther, 1864 - Pirapitinga & Flu & [168] \\
\hline Colossoma macropomum (Cuvier, 1818) - Black-finned colossoma & Paralysis of arms and legs & {$[8,70]$} \\
\hline Hydrolycus scomberoides (Cuvier, 1816) - Vampire characin & Earache & {$[8,188]$} \\
\hline Salminus brasiliensis (Cuvier, 1816)- Jaw characin & Unspecified & [179] \\
\hline Clupeidae & & \\
\hline Opisthonema oglinum (Lesueur, 1818) - Atlantic thread herring & Alcoholism & [7-9] \\
\hline Dasyatidae & & \\
\hline Dasyatis guttata (Bloch \& Schneider, 1801) - Longnose stingray & $\begin{array}{l}\text { Asthma, Injuries caused by } \\
\text { fish species, burns }\end{array}$ & [7-9] \\
\hline $\begin{array}{l}\text { Dasyatis marianae (Gomes, Rosa \& Gadig, 2000) - Brazilian large- } \\
\text { eyed stingray }\end{array}$ & $\begin{array}{l}\text { Asthma, Injuries caused by } \\
\text { fish species, burns }\end{array}$ & $[7-9])$ \\
\hline Doradidae & & \\
\hline Franciscodoras marmoratus (Reinhardt, 1874) - Urutu & $\begin{array}{l}\text { Injuries caused by fish } \\
\text { species }\end{array}$ & [149] \\
\hline Lithodoras dorsalis (Valenciennes, 1840) - Bacu Pedra & Swelling & [7-9] \\
\hline $\begin{array}{l}\text { Megalodoras uranoscopus (Eigenmann \& Eigenmann, 1888) - } \\
\text { Catfish }\end{array}$ & Rheumatism & [188] \\
\hline Platydoras costatus (Linnaeus, 1758) - Catfish & Rheumatism & [188] \\
\hline Pterodoras granulosus (Valenciennes, 1821) - Catfish & Rheumatism & [188] \\
\hline Oxydoras niger (Valenciennes, 1821) - Catfish & Rheumatism & {$[82,188]$} \\
\hline Echeneidae & & \\
\hline Echeneis naucrates Linnaeus, 1758 - Live sharksucker & Asthma, bronchits & {$[8,114,162,164]$} \\
\hline
\end{tabular}


Table 1 Medicinal animals and its respective uses in popular medicine in Latin America (Continued)

\section{Electrophoridae}

Electrophorus electricus (Linnaeus, 1766) - Electric eel

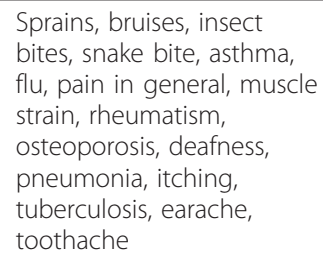

Sprains, bruises, insect

bites, snake bite, asthma,

flu, pain in general, muscle

strain, rheumatism,

osteoporosis, deafness,

pneumonia, itching,

tuberculosis, earache,

toothache

$[7-9,31,114,162,164,188]$

\section{Erythrinidae}

Erythrinus erythrinus (Bloch \& Schneider, 1801) - Red (hi-fin) Wolf Asthma fish

Hoplias malabaricus (Bloch, 1794) - Trahira

\section{Ophthalmological problems, rheumatism, cataracts, wounds, snake bite, conjunctivitis, stroke, thrombosis, asthma, toothache, fever, earache, diarrhoea, deafness, boils, bleedings, Alcoholism, tetanus, sore throat, itching, sprains, leucoma}

\begin{tabular}{|c|c|c|}
\hline Hoplias lacerdae Miranda Ribeiro, 1908 Giant trahira & Leucoma & {$[71]$} \\
\hline \multicolumn{3}{|l|}{ Gadidae } \\
\hline Gadus morhua Linnaeus, 1758 - Atlantic cod & $\begin{array}{l}\text { Boils, backache and } \\
\text { rheumatism }\end{array}$ & {$[66,228]$} \\
\hline \multicolumn{3}{|l|}{ Ginglymostomatidae } \\
\hline Ginglymostoma cirratum (Bonnaterre, 1788) - Nurse shark & Rheumatism & {$[7-9]$} \\
\hline \multicolumn{3}{|l|}{ Heptapteridae } \\
\hline Pimelodella brasiliensis (Steindachner, 1876) - Mandim & $\begin{array}{l}\text { Injuries caused by that fish } \\
\text { species }\end{array}$ & {$[168]$} \\
\hline \multicolumn{3}{|l|}{ Holocentridae } \\
\hline Holocentrus adscensionis (Osbeck, 1765) - Squirrelfish & Wounds & {$[68]$} \\
\hline \multicolumn{3}{|l|}{ Megalopidae } \\
\hline Megalops atlanticus (Valenciennes, 1847) - Tarpon & $\begin{array}{l}\text { Stroke, headache, asthma, } \\
\text { shortness of breath, } \\
\text { thrombosis, chest pain, } \\
\text { injuries caused by bang }\end{array}$ & {$[7-9,97,114]$} \\
\hline \multicolumn{3}{|l|}{ Monacanthidae } \\
\hline Stephanolepis hispidus (Linnaeus, 1766) - Common filefish & Unspecified & [111] \\
\hline \multicolumn{3}{|l|}{ Muraenidae } \\
\hline Gymnothorax funebris Ranzani, 1840 - Green moray & Bleeding & {$[7-9]$} \\
\hline Gymnothorax moringa (Cuvier, 1829) - Spotted moray & Bleeding & {$[7-9]$} \\
\hline Gymnothorax vicinus (Castelnau, 1855) - Purplemouth moray & Bleeding & {$[7-9]$} \\
\hline \multicolumn{3}{|l|}{ Odontaspididae } \\
\hline Eugomphodus taurus (Rafinesque 1810) - Cação-mangona & Unspecified & [111] \\
\hline \multicolumn{3}{|l|}{ Engraulidae } \\
\hline Anchoviella lepidentostole (Fowler, 1911) & Unspecified & {$[80]$} \\
\hline \multicolumn{3}{|l|}{ Pristigasteridae } \\
\hline Pellona flavipinnis Valenciennes, 1836 - Yellowfin river pellona & Unspecified & {$[80]$} \\
\hline \multicolumn{3}{|l|}{ Scombridae } \\
\hline Scomberomorus cavalla Cuvier, 1829 - Kingfish & Unspecified & {$[80]$} \\
\hline \multicolumn{3}{|l|}{ Myliobatidae } \\
\hline Aetobatus narinari (Euphrasen, 1790) - Spotted eagle ray & $\begin{array}{l}\text { Asthma, injuries caused by } \\
\text { fish species, burns, } \\
\text { haemorrhage }\end{array}$ & {$[7-9]$} \\
\hline
\end{tabular}

\section{[97]}

$[7-9,31,63,97,114,126,162,164,188]$ 
Table 1 Medicinal animals and its respective uses in popular medicine in Latin America (Continued)

\begin{tabular}{|c|c|c|}
\hline Narcinidae & & \\
\hline Narcine brasiliensis (Olfers, 1831) - Brazilian electric Ray & Toothache & {$[111,114,162]$} \\
\hline Ogcocephalidae & & \\
\hline Ogcocephalus vespertilio (Linnaeus, 1758) - Batfish & Asthma, bronchits & {$[7-9,68]$} \\
\hline Ogcocephalus nasutus (Cuvier, 1829) - Batfish & Asthma & [79] \\
\hline Loricariidae & & \\
\hline Hypostomus plecostomus (Linnaeus, 1758) & Asthma & [79] \\
\hline Carangidae & & \\
\hline Hemicaranx amblyrhynchus (Cuvier, 1833) & $\begin{array}{l}\text { To accelerate recovery after } \\
\text { parturition }\end{array}$ & [79] \\
\hline Osteoglossidae & & \\
\hline Arapaima gigas (Schinz, 1822) - Giant arapaima & Asthma, pneumonia & [188] \\
\hline Osteoglossum ferreirai (Kanazawa, 1966) - Aruanã & Dermatogical problems & {$[87]$} \\
\hline Pimelodidae & & \\
\hline $\begin{array}{l}\text { Phractocephalus hemioliopterus (Bloch \& Schneider, 1801) - Redtail } \\
\text { catfish }\end{array}$ & $\begin{array}{l}\text { Asthma, wounds, hernia, } \\
\text { burns in the skin, } \\
\text { rheumatism, flu, cough, } \\
\text { pneumonia }\end{array}$ & {$[87,188]$} \\
\hline $\begin{array}{l}\text { Pseudoplatystoma corruscans (Spix \& Agassiz, 1829) - Spotted } \\
\text { sorubim }\end{array}$ & Flu & [168] \\
\hline Pseudoplatystoma fasciatum (Lunnaeus, 1776) - Tiger catfish & Cold & {$[188]$} \\
\hline Pseudoplatystoma tigrinum (Valenciennes, 1840) - Catfish & Unspecified & [179] \\
\hline Sorubimichthys planiceps (Spix \& Agassiz, 1829) & Leishmaniosis, tuberculosis & [188] \\
\hline Zungaro zungaro((Humboldt, 1821) - Black manguruyu & $\begin{array}{l}\text { Asthma, toothache, } \\
\text { earache, wounds, athlete's } \\
\text { foot, burns in the skin, } \\
\text { rheumatism, flu }\end{array}$ & [188] \\
\hline Rhamdia quelen (Quoy \& Gaimard, 1824) & Tonic & {$[177]$} \\
\hline Potamotrygonidae & & \\
\hline Paratrygon aiereba (Müller \& Henle, 1841) - Discus Ray & $\begin{array}{l}\text { Asthma, hernia, flu, } \\
\text { pneumonia, cough, } \\
\text { earache, burns }\end{array}$ & [188] \\
\hline $\begin{array}{l}\text { Potamotrygon hystrix (Müller \& Henle, 1834) - Porcupine river } \\
\text { stingray }\end{array}$ & $\begin{array}{l}\text { Asthma, hernia, flu, } \\
\text { pneumonia, cough, } \\
\text { earache, burns }\end{array}$ & {$[70,188]$} \\
\hline $\begin{array}{l}\text { Potamotrygon motoro (Müller \& Henle, 1841) - Ocellate river } \\
\text { stingray }\end{array}$ & $\begin{array}{l}\text { Asthma, hernia, flu, } \\
\text { pneumonia, cough, } \\
\text { earache, burns }\end{array}$ & [188] \\
\hline Potamotrygon orbignyi (Castelnau, 1855) & $\begin{array}{l}\text { Injuries caused by fish } \\
\text { species }\end{array}$ & {$[7-9]$} \\
\hline Plesiotrygon iwamae Rosa, Castello \& Thorson, 1987 & $\begin{array}{l}\text { Injuries caused by fish } \\
\text { species, wounds, cracks in } \\
\text { the sole of the feet }\end{array}$ & {$[7-9]$} \\
\hline Potamotrygon signata German, 1913 & Unspecified & {$[80]$} \\
\hline Pristidae & & \\
\hline Pristis pectinata Latham, 1794 - Smalltooth sawfish & $\begin{array}{l}\text { Asthma, rheumatism, } \\
\text { arthritis }\end{array}$ & {$[7-9]$} \\
\hline $\begin{array}{l}\text { Pristis perotteti Müller \& Henle, } 1841 \text { - Largetooth sawfish, } \\
\text { espadarte }\end{array}$ & $\begin{array}{l}\text { Asthma, rheumatism and } \\
\text { arthritis }\end{array}$ & {$[7-9]$} \\
\hline Prochilodontidae & & \\
\hline Prochilodus argenteus Spix \& Agassiz, 1829 - & $\begin{array}{l}\text { To avoid swelling of the } \\
\text { breast feeding, mycosis }\end{array}$ & {$[168]$} \\
\hline Prochilodus lineatus (Valenciennes, 1836) & Unspecified & [160] \\
\hline
\end{tabular}


Table 1 Medicinal animals and its respective uses in popular medicine in Latin America (Continued)

\begin{tabular}{|c|c|c|}
\hline Prochilodus nigricans Spix \& Agassiz, 1829 - Black prochilodus & $\begin{array}{l}\text { Chilblain, skin burns, } \\
\text { wounds, rheumatism, eye } \\
\text { pains }\end{array}$ & {$[157,188]$} \\
\hline Prochilodus marggravii (Walbaum, 1792) & Unspecified & {$[168]$} \\
\hline \multicolumn{3}{|l|}{ Rajidae } \\
\hline Atlantoraja cyclophora Regan, 1903 - Eyespot skate & Haemorrhage after delivery & [68] \\
\hline \multicolumn{3}{|l|}{ Serrasalmidae } \\
\hline Mylossoma duriventre (Cuvier, 1818) & Venereal disease & [188] \\
\hline Serrasalmus brandtii (Lütken, 1875) - White piranha & $\begin{array}{l}\text { Inflammations, sexual } \\
\text { impotence }\end{array}$ & {$[71,97,126]$} \\
\hline Serrasalmus rhombeus Linnaeus 1766 & Unspecified & {$[80,82]$} \\
\hline \multicolumn{3}{|l|}{ Sciaenidae } \\
\hline Cynoscion acoupa (Lacepède, 1801) - Acoupa weakfish & Renal failure & {$[7-9,31]$} \\
\hline Cynoscion leiarchus (Cuvier, 1830) - Smooth weakfish & Renal failure & {$[7-9,31]$} \\
\hline Micropogonias furnieri (Desmarest, 1823) - Whitemouth croaker & $\begin{array}{l}\text { Injuries caused by fish } \\
\text { species, cough, asthma, } \\
\text { bronchits }\end{array}$ & {$[8,85,114,162]$} \\
\hline Pachyurus francisci (Cuvier, 1830) - San Francisco croaker & $\begin{array}{l}\text { Asthma, urinary } \\
\text { incontinence, backache }\end{array}$ & [168] \\
\hline Plagioscion surinamensis (Bleeker, 1873) - Bashaw & $\begin{array}{l}\text { Urinary disorders, } \\
\text { haemorrhage, snake bites }\end{array}$ & [188] \\
\hline $\begin{array}{l}\text { Plagioscion squamosissimus (Heckel, 1840) - South american silver } \\
\text { croaker }\end{array}$ & $\begin{array}{l}\text { Urinary disorders, } \\
\text { haemorrhage, snake bites }\end{array}$ & [188] \\
\hline \multicolumn{3}{|l|}{ Sparidae } \\
\hline Calamus penna (Valenciennes, 1830) - Sheepshead porgy & Asthma & {$[162]$} \\
\hline \multicolumn{3}{|l|}{ Synbranchidae } \\
\hline Synbranchus marmoratus Bloch, 1795 - Marbled swamp eel & Bronchits & {$[114,162]$} \\
\hline \multicolumn{3}{|l|}{ Syngnathidae } \\
\hline Hippocampus erectus Perry, 1810 - Horsefish & Asthma & {$[7-9,31,75]$} \\
\hline Hippocampus reidi (Ginsburg, 1933) - Longsnout seahorse & $\begin{array}{l}\text { Asthma, edema, bronchits, } \\
\text { haemorrhage, } \\
\text { haemorrhage in women, } \\
\text { disorders after parturition, } \\
\text { gastritis, tuberculosis, to } \\
\text { prevent abortion }\end{array}$ & {$[7-9,31,63,68,85,164]$} \\
\hline Hippocampus ingens Girard, 1858 & Unspecified & [76] \\
\hline Hippocampus spinosissimus Weber, 1913 & Unspecified & [76] \\
\hline Hippocampus trimaculatus Leach, 1814 & Unspecified & [76] \\
\hline \multicolumn{3}{|l|}{ Tetraodontidae } \\
\hline Colomesus psittacus (Bloch \& Schneider, 1801) - Banded puffer & $\begin{array}{l}\text { Breast cancer, backache, } \\
\text { warts }\end{array}$ & {$[7-9,31]$} \\
\hline Sphoeroides testudineus (Linnaeus, 1758) - Checkered puffer & Rheumatism & {$[63,162]$} \\
\hline \multicolumn{3}{|l|}{ Trichiuridae } \\
\hline Trichiurus lepturus Linnaeus, 1758 - Largehead hairtail & Asthma & {$[8]$} \\
\hline \multicolumn{3}{|l|}{ Gymnotidae } \\
\hline Gymnotus carapo Linnaeus, 1758 - Banded knifefish & Unspecified & {$[179]$} \\
\hline \multicolumn{3}{|l|}{ Urolophidae } \\
\hline $\begin{array}{l}\text { Urotrygon microphthalmum (Delsman, 1941) - Smalleyed round } \\
\text { stingray }\end{array}$ & $\begin{array}{l}\text { Asthma, Injuries caused by } \\
\text { fish species, burns }\end{array}$ & {$[7-9,31]$} \\
\hline \multicolumn{3}{|l|}{ AMPHIBIANS } \\
\hline \multicolumn{3}{|l|}{ Bufonidae } \\
\hline Rhinella schneideri (Werner, 1894) - Cururu toad & $\begin{array}{l}\text { Urinary incontinence, } \\
\text { dental caries, cancer, } \\
\text { wounds, boils, erysipelas } \\
\text { acne, to induce abortion }\end{array}$ & {$[97,162]$} \\
\hline
\end{tabular}


Table 1 Medicinal animals and its respective uses in popular medicine in Latin America (Continued)

\begin{tabular}{|c|c|c|}
\hline Rhinella marina (Linnaeus, 1758) - toad & Scorpion sting, erysipelas & {$[87,152,158]$} \\
\hline Rhinella jimi (Stevaux, 2002)- toad & Gastritis, cancer & {$[81]$} \\
\hline Rhinella icterica (Spix, 1824) & Unspecified & {$[80]$} \\
\hline Bufo bocourti Brocchi, 1877 - toad & Rabies, AIDS & {$[38]$} \\
\hline Bufo macrocristatus Firschein and Smith, 1957 - toad & Unspecified & {$[38]$} \\
\hline Incilius valliceps (Wiegmann, 1833) & Unspecified & {$[125]$} \\
\hline \multicolumn{3}{|l|}{ Leptodactylidae } \\
\hline $\begin{array}{l}\text { Leptodactylus labyrinthicus (Spix, 1824) - South american pepper } \\
\text { frog }\end{array}$ & $\begin{array}{l}\text { Earache, rheumatism, joint } \\
\text { pain, cancer, sore throat }\end{array}$ & {$[97,162]$} \\
\hline Leptodactylus vastus Lutz, 1930-frog & Sore throat & {$[66]$} \\
\hline Leptodactylus troglodytes (A. Lutz, 1926) - Jia & Unspecified & {$[4]$} \\
\hline Eleutherodactylus laticeps (Duméril, 1853) & Unspecified & {$[38]$} \\
\hline Eleutherodacty/us glaucus Lynch, 1967 & Unspecified & {$[38]$} \\
\hline \multicolumn{3}{|l|}{ Ranidae } \\
\hline Rana maculata Brocchi, 1877 & Rabies & {$[38]$} \\
\hline Rana berlandieri Baird, 1859 & & [38] \\
\hline Lithobates montezumae (Baird, 1854) & Unspecified & {$[125]$} \\
\hline Lithobates spectabilis (Hillis and Frost, 1985) & Unspecified & [125] \\
\hline \multicolumn{3}{|l|}{ Hylidae } \\
\hline Hyla chaneque Duellman, 1961 & Unspecified & {$[38]$} \\
\hline Hyla venulosa (Laurenti, 1768) & & {$[60]$} \\
\hline Phyllomedusa bicolor (Boddaert, 1772) & Rheumatism, diabetes & [122] \\
\hline Trachycephalus resinifictrix (Goeldi, 1907) & Unspecified & {$[82]$} \\
\hline Phyllomedusa burmeisteri Boulenger, 1882 & Unspecified & [183] \\
\hline \multicolumn{3}{|l|}{ Microhylidae } \\
\hline Hypopachus barberi Schmidt, 1939 & Unspecified & {$[38]$} \\
\hline \multicolumn{3}{|l|}{ Ceratophryidae } \\
\hline Telmatobius culeus (Garman, 1876) & Impotence & {$[180]$} \\
\hline \multicolumn{3}{|l|}{ REPTILES } \\
\hline \multicolumn{3}{|l|}{ Liolaemidae } \\
\hline Liolaemus pantherinus Pellegrin 1909 & Fractures, wounds & {$[65,189]$} \\
\hline Liolaemus alticolor Barbour 1909 & Fractures, wounds & [189] \\
\hline \multicolumn{3}{|l|}{ Gekkonidae } \\
\hline $\begin{array}{l}\text { Hemidactylus mabouia (Moreau de Jonnes, 1818) - Afro-American } \\
\text { house gecko }\end{array}$ & Sore throat & $[7-9,31,164])$ \\
\hline Gonatodes hasemani Griffin 1917 & Unspecified & [174] \\
\hline \multicolumn{3}{|l|}{ Iguanidae } \\
\hline Iguana iguana (Linnaeus, 1758) - Common iguana & $\begin{array}{l}\text { Earache, erysipelas, asthma, } \\
\text { rheumatism, edema, } \\
\text { abscesses, joint pain, } \\
\text { wounds, acne, athlete's } \\
\text { foot, sore throat, swelling, } \\
\text { burn, tumour, to suck a } \\
\text { splinter out of skin or flesh, } \\
\text { boil, injuries caused by the } \\
\text { spines of the 'arraia' and } \\
\text { others fishes, inflammation, } \\
\text { hernia, body aches, corisa, } \\
\text { womb disorders, menstrual } \\
\text { cramps }\end{array}$ & $[7-9,31,97,108,164,166])$ \\
\hline Ctenosaura pectinata Wiegmann 183 & Unspecified & {$[94,105]$} \\
\hline Ctenosaura similis GRAY 1831 & Unspecified & {$[77]$} \\
\hline
\end{tabular}


Table 1 Medicinal animals and its respective uses in popular medicine in Latin America (Continued)

\section{Polychrotidae}

\begin{tabular}{lll}
\hline Polychrus acutirostris Spix 1825 & Impotence & [126] \\
\hline Polychrus marmoratus Linnaeus 175 & Impotence & [126] \\
\hline Norops fuscoauratus D'orbigny 1837 & Unspecified & [174]
\end{tabular}

\section{Teiidae}

Ameiva ameiva (Linnaeus, 1758) - Lizard

Inflammation, dermatitis,

$[87,97,108,174]$ bites

Kentropyx pelviceps Cope 1868

Unspecified

[174]

Cnemidophorus gr. ocellifer (Spix, 1825) - Lizard

Inflammation, dermatitis, venereal diseases, snake bites

Tupinambis merianae (Duméril \& Bibron, 1839) - Lizard

Earache, deafness, rheumatism, erysipelas, skin thorns and wounds, respiratory diseases, sore throat, snake bite, asthma, tumour, swelling, infection, bronchits, perforation, oftalmological problems

rheumatism, erysipelas, dermatitis, snake bites, asthma, tetanus, earache, thrombosis, wounds, panaris, swelling, herpes zoster, irritation when milk teeth are erupting, jaundice, inflammation, tumour, sore throat, infection, bronchits, injuries caused by the spines of the 'arraia', pain relief in injuries caused by snake bites, toothache, suck a splinter out of skin or fresh, headache, cough, stroke, coarse throat

Tupinambis rufescens Günther 1871 - tegu Cuts, snake bites, cold

\section{Phrynosomatidae}

\begin{tabular}{lll}
\hline Sceloporus serrifer Cope 1866 & Unspecified & {$[38]$} \\
\hline Sceloporus taeniocnemis Cope 1885 & Unspecified & {$[38]$} \\
\hline Sceloporus acanthinus Bocourt 1873 & Unspecified & {$[91]$} \\
\hline Sceloporus spinosus Wiegmann 1828 & Unspecified & {$[91]$} \\
\hline Sceloporus grammicus Wiegmann 1828 & Diarrhea & {$[103]$} \\
\hline Phrynosoma orbiculare (Duméril \& Bocourt 1870) & Unspecified & {$[91]$} \\
\hline
\end{tabular}

\section{Anguidae}

\begin{tabular}{lll}
\hline Abronia Iythrochila Smith \& Alvarez Del Toro 1963 & Unspecified & [38] \\
\hline Mesaspis moreletii Bocourt 1871 & Unspecified & [38] \\
\hline Tropiduridae & & \\
\hline Tropidurus hispidus (Spix, 1825) - Lizard & Alcoholism, & [7-9,31] \\
& dermatomycosis, warts, & \\
& abscesses, boils, sore & \\
& throat, erysipelas, healing & \\
& of umbilical cord of & \\
\hline Tropidurus semitaeniatus (Spix, 1825) - Lizard & newborn baby & Measles, asthma, \\
& alcoholism, & {$[8,62,97,162]$}
\end{tabular}


Table 1 Medicinal animals and its respective uses in popular medicine in Latin America (Continued)

\begin{tabular}{|c|c|c|}
\hline Tropidurus torquatus (Wied, 1820) - Lizard & Chicken pox & {$[7-9,31,62,71,97,115,162]$} \\
\hline Uranoscodon superciliosus (Linnaeus, 1758) & As a sedative & {$[8,62,69,131]$} \\
\hline \multicolumn{3}{|l|}{ Boidae } \\
\hline Boa constrictor (Linnaeus, 1758) - Boa & $\begin{array}{l}\text { Rheumatism, lung disease, } \\
\text { thrombosis, boils, } \\
\text { tuberculosis, stomach ache, } \\
\text { edema, snake bite, cancer, } \\
\text { pains, swelling, to prevent } \\
\text { abort, pain in the body, } \\
\text { inflammation, athlete's } \\
\text { foot, calluses, tumours, } \\
\text { cracks in the sole of the } \\
\text { feets, goiter, sore throat, } \\
\text { arthrosis, insect sting, dog } \\
\text { bite, erysipelas, asthma, } \\
\text { neck strain, strain muscle, } \\
\text { backache, contusions and } \\
\text { muscular pain }\end{array}$ & {$[7-10,29,31,62,70,97,121,186,188]$} \\
\hline Corallus caninus (Linnaeus, 1758) - American emerald tree boa & $\begin{array}{l}\text { Pain relief caused by sting } \\
\text { of animals }\end{array}$ & {$[8,29,62]$} \\
\hline Corallus hortolanus (Linnaeus, 1758) - Snake & $\begin{array}{l}\text { To assist in removing } \\
\text { spines or other sharp } \\
\text { structures from the skin, } \\
\text { rheumatism }\end{array}$ & {$[8,29,62]$} \\
\hline Eunectes murinus (Linnaeus, 1758) - Anaconda & $\begin{array}{l}\text { Wounds, skin problems, } \\
\text { bruises, sprains, arthrosis, } \\
\text { rheumatism, boils, sexual } \\
\text { impotence, headache, sore } \\
\text { throat, thrombosis, } \\
\text { swelling, tumour, asthma, } \\
\text { muscle strain, numbness, } \\
\text { syphilis, to reduce pain, } \\
\text { luxation }\end{array}$ & {$[8,29,62,71]$} \\
\hline Eunectes notaeus (Cope, 1862)- Sucuri & Unspecified & {$[160]$} \\
\hline Epicrates cenchria (Linnaeus, 1758) - Brazilian rainbow boa & $\begin{array}{l}\text { Rheumatism, pain in } \\
\text { articulations, snake bite, } \\
\text { sore throat }\end{array}$ & {$[8,29,62,71,97]$} \\
\hline \multicolumn{3}{|l|}{ Colubridae } \\
\hline Leptophis ahetula (Linnaeus, 1758) - Parrot snake & $\begin{array}{l}\text { Pain relief caused by sting } \\
\text { of animals }\end{array}$ & {$[8,29,62]$} \\
\hline Mastigodryas bifossatus (Raddi, 1820) - Rio tropical racer & Snake bites & {$[8,29,62,108]$} \\
\hline Pituophis lineaticollis Cope 1861 & $\begin{array}{l}\text { Post partum cold, pains } \\
\text { corporeal }\end{array}$ & {$[40]$} \\
\hline Oxyrhopus trigeminus Duméril, Bibron \& Duméril, 1854 & Rheumatism & {$[8,29,62,108]$} \\
\hline Oxyrhopus formosus Wied 1820 & Unspecified & {$[174]$} \\
\hline Oxyrhopus melanogenys Tschudi 1845 & Unspecified & {$[174]$} \\
\hline Spilotes pullatus (Linnaeus, 1758) - Tiger snake & Insects bite, snake bite & {$[8,29,62,108]$} \\
\hline Tachymenis peruviana Wiegmann, 1835 & $\begin{array}{l}\text { Pain in the bones, pain in } \\
\text { kidneys and to treat } \\
\text { inflamations, tootache and } \\
\text { scare, fractures }\end{array}$ & {$[148,189]$} \\
\hline Drymobius margaritiferus Schlegel 1837 & $\begin{array}{l}\text { Mail aire, pain in bones, } \\
\text { rheumatism }\end{array}$ & {$[38,148]$} \\
\hline Chironius carinatus (Linnaeus, 1758) & Infertility & {$[151]$} \\
\hline Chironius grandisquamis Peters 1869 & Unspecified & {$[174]$} \\
\hline Lampropeltis triangulum Lacépède 1789 & Unspecified & {$[38,148]$} \\
\hline Imantodes cenchoa Linnaeus 1758 & Unspecified & {$[174]$} \\
\hline Leptodeira annulata (Linnaeus, 1758) & Unspecified & {$[174]$} \\
\hline
\end{tabular}


Table 1 Medicinal animals and its respective uses in popular medicine in Latin America (Continued)

\section{Crotalidae}

Caudisona durissa (Linnaeus, 1758) - Neotropical rattlesnake

Asthma, snake bite, thrombosis, wounds, luxation, rheumatism, pain

in the legs, erysipelas, deafness, epilepsy, skin diseases, tuberculosis, hanseniasis, backache, tumour, boil, headache, earache, osteoporosis, sore throat, toothache, insects bite, irritation when milk teeth are erupting, cancer, to accelerate parturition, pain in the body, avoid pregnancy, mail aire, swellings, bone aches, gastritis, eczema

\begin{tabular}{ll}
\hline Crotalus polystictus Cope 1865 & Unspecified \\
\hline Crotalus transversus Taylor 1944 & Unspecified \\
\hline Crotalus triseriatus Wagler 1830 & Unspecified \\
\hline Crotalus durissus Linnaeus 1758 & Wounds, disorders in \\
& parturition, lepra, cancer, \\
& acne \\
\hline Crotalus atrox Baird \& Girard 1853 & $\begin{array}{l}\text { Pneumonia, muscular pain } \\
\text { sight, sore throat, } \\
\text { gangrene, varicose veins, } \\
\text { ulcer }\end{array}$ \\
\hline
\end{tabular}

Crotalus basiliscus COPE 1864

\section{Elapidae}

Micrurus spixii Wagler 1824

Micrurus ibiboboca (Merrem, 1820)

\section{Viperidae}

\begin{tabular}{lc}
\hline Bothrops leucurus Wagler, 1824 - Lance head & Tur \\
\hline Bothrops asper Garman 1883 & Uns \\
\hline Bothrops atrox Linnaeus 1758 & Uns \\
\hline Lachesis muta (Linnaeus, 1766) - Bushmaster
\end{tabular}

Lachesis muta (Linnaeus, 1766) - Bushmaster

\begin{tabular}{l} 
Cerrophidion tzotzilorum Campbell 1985 \\
Atropoides nummifer Rüppell 1845 \\
\hline Dipsadidae \\
\hline Philodryas olfersii Lichenstein, 1823 \\
\hline Thamnodynastes strigatus (Günther, 1858) \\
\hline Chelidae \\
Chelus fimbriatus (Schneider, 1783) - Matá-matá \\
Phrynops geoffroanus (Schweigger, 1812) - Geoffroy's side-necked \\
turtle
\end{tabular}
snake bite Unspecified

[78]

\begin{tabular}{ll} 
& \\
Unspecified & {$[174]$} \\
\hline Unspecified & {$[174]$} \\
\hline Rheumatism, snake bite & {$[62,108,139]$} \\
\hline
\end{tabular}

\section{[125]}

[125]

[125]

$[38,105]$

$[62,108,139]$

Tumour, boils $\quad[62,108,139]$

Unspecified $\quad[38,148,152]$

Unspecified [80,185]

Rheumatism, swelling, [8]

tumour, boil, insects bite,

\begin{tabular}{ll} 
Rheumatism, mail aire & {$[38,148]$} \\
\hline Unspecified & {$[38,148]$}
\end{tabular}

Unspecified [80]

Snake bite [151]

Impotence [82,87,153]

Asthma, sore throat, $\quad[7-9,71]$

swelling, earache,

rheumatism, arthrosis, healing of umbilical cord of newborn baby, mumps, ingrown nail, eczema, articulation problems, wounds 
Table 1 Medicinal animals and its respective uses in popular medicine in Latin America (Continued)

\begin{tabular}{|c|c|c|}
\hline $\begin{array}{l}\text { Mesoclemmys tuberculata (Luederwaldt, 1926) - Tuberculate } \\
\text { toadhead turtle }\end{array}$ & $\begin{array}{l}\text { Rheumatism, discharge, } \\
\text { thrombosis, bronchits, } \\
\text { diarrhoea, haemorrhag, } \\
\text { asthma, sore throat, } \\
\text { hoarseness }\end{array}$ & {$[62,97,108,161]$} \\
\hline Mauremys leprosa Schweigger, 1812 & Unspecified & {$[80]$} \\
\hline \multicolumn{3}{|l|}{ Cheloniidae } \\
\hline Caretta caretta (Linnaeus, 1758) - Loggerhead turtle & $\begin{array}{l}\text { Injuries caused by bang, } \\
\text { toothache, diabetes, } \\
\text { headache, backache, } \\
\text { wounds, cough, bronchits, } \\
\text { asthma, thrombosis, } \\
\text { rheumatism, stroke, } \\
\text { hoarseness, flu, backache, } \\
\text { earache, sore throat, } \\
\text { swelling }\end{array}$ & {$[7-9,62,139,142,162]$} \\
\hline Chelonia mydas (Linnaeus, 1758) - Green sea turtle & $\begin{array}{l}\text { Injuries caused by bang, } \\
\text { toothache, diabetes, } \\
\text { headache, backache, } \\
\text { wounds, cough, bronchits, } \\
\text { asthma, flu, thrombosis, } \\
\text { rheumatism, stroke, } \\
\text { hoarseness, earache, sore } \\
\text { throat, swelling, whooping } \\
\text { cough, arthritis, erysipelas, } \\
\text { boil, arthrosis, inflammation }\end{array}$ & {$[7-9,62,68,139,142]$} \\
\hline Eretmochelys imbricata (Linnaeus, 1766) - Atlantic hawksbill & $\begin{array}{l}\text { Injuries caused by bang, } \\
\text { toothache, diabetes, } \\
\text { headache, backache, } \\
\text { wounds, cough, bronchits, } \\
\text { asthma, thrombosis, stroke, } \\
\text { hoarseness, flu, } \\
\text { rheumatism, earache, sore } \\
\text { throat, swelling }\end{array}$ & {$[7-9,62,139,142,162]$} \\
\hline Lepidochelys olivacea (Eschscholtz, 1829) & $\begin{array}{l}\text { Injuries caused by bang, } \\
\text { toothache, diabetes, } \\
\text { headache, backache, } \\
\text { wounds, cough, flu, } \\
\text { bronchitis, asthma, } \\
\text { thrombosis, rheumatism, } \\
\text { stroke, hoarseness }\end{array}$ & {$[62,114,119,162]$} \\
\hline \multicolumn{3}{|l|}{ Dermochelyidae } \\
\hline Dermochelys coriacea (Vandelli, 1761) - Leatherback turtle & $\begin{array}{l}\text { Rheumatism, earache, sore } \\
\text { throat, swelling }\end{array}$ & {$[7-9,62,139,142]$} \\
\hline \multicolumn{3}{|l|}{ Geoemydidae } \\
\hline Rhinoclemmys punctularia (Daudin, 1802) - Spot-legged turtle & $\begin{array}{l}\text { Wounds, tumour, } \\
\text { erysipelas, earache, } \\
\text { rheumatism }\end{array}$ & {$[7-9,62,139,142]$} \\
\hline \multicolumn{3}{|l|}{ Podocnemididae } \\
\hline Podocnemis expansa (Schweiger,1812) - Amazon river turtle & $\begin{array}{l}\text { Inflammation, acne, } \\
\text { tumour, boil, rheumatism, } \\
\text { pterygium, skin spots, } \\
\text { backache, earache, } \\
\text { arthrosis, arthritis, swelling, } \\
\text { wrinkle }\end{array}$ & {$[7-9,62,139,141]$} \\
\hline Podocnemis unifilis (Troschel, 1848) - Yellow-spotted river turtle & $\begin{array}{l}\text { Wounds, tumour, } \\
\text { erysipelas, earache, } \\
\text { rheumatism }\end{array}$ & {$[7-9,62,139,188]$} \\
\hline $\begin{array}{l}\text { Podocnemis sextuberculata Cornalia, } 1849 \text { - Six-tubercled Amazon } \\
\text { River turtle }\end{array}$ & Blackhead, acne & {$[62,69]$} \\
\hline Peltocephalus dumeriliana Schweigger 1812 & Blackhead, acne & {$[62,69]$} \\
\hline
\end{tabular}


Table 1 Medicinal animals and its respective uses in popular medicine in Latin America (Continued)

\begin{tabular}{|c|c|c|}
\hline \multicolumn{3}{|l|}{ Testudinidae } \\
\hline Chelonoidis carbonaria (Spix, 1824) - Red-footed tortoise & $\begin{array}{l}\text { Catarrh, erysipelas, } \\
\text { bronchitis, to stop the } \\
\text { sensation to getting thirsty, } \\
\text { asthma }\end{array}$ & {$[8,9,31,63,115,162,164]$} \\
\hline Chelonoidis denticulata (Linnaeus, 1766) - Yellowfooted tortoise & $\begin{array}{l}\text { Sore throat, rheumatism, } \\
\text { hernia, wounds, } \\
\text { leishmaniosis, varicocele, } \\
\text { earache, part of woman's } \\
\text { body, asthma, pains }\end{array}$ & {$[8,9,31,87,157]$} \\
\hline Gopherus flavomarginatus Legler 1959 & Arthritis & [103] \\
\hline \multicolumn{3}{|l|}{ Kinosternidae } \\
\hline Staurotypus triporcatus Wiegmann 1828 & Unspecified & [93] \\
\hline Kinosternon scorpioides Linnaeus 1766 & $\begin{array}{l}\text { Hermaphroditism, malaria, } \\
\text { tuberculosis }\end{array}$ & {$[38,62]$} \\
\hline Kinosternon integrum Le Conte 1854 & Smallpox & [105] \\
\hline \multicolumn{3}{|l|}{ Alligatoridae } \\
\hline Caiman crocodilus (Linnaeus, 1758) - Common cayman & $\begin{array}{l}\text { Asthma, stroke, bronchitis, } \\
\text { backache, earache, } \\
\text { rheumatism, thrombosis, } \\
\text { sexual impotence, snake } \\
\text { bites, evil eye, irritation } \\
\text { when milk teeth are } \\
\text { erupting, discharge, } \\
\text { swelling, scratch, athlete's } \\
\text { foot, ophthalmological } \\
\text { problems, sore throat, } \\
\text { amulet used as a } \\
\text { protection against snake } \\
\text { bite, hernia, prostate } \\
\text { problems, infection, } \\
\text { inflammation, epilepsy }\end{array}$ & {$[7-9,30,62,63,108,139,162-164,166,188]$} \\
\hline Caiman latirostris (Daudin, 1801) - Cayman & $\begin{array}{l}\text { Asthma, sore throat, } \\
\text { amulet used as a } \\
\text { protection against snake } \\
\text { bite, rheumatism, irritation } \\
\text { when milk teeth are } \\
\text { erupting, hernia, prostate } \\
\text { problems }\end{array}$ & {$[7-9,62,139]$} \\
\hline Caiman yacare Daudin 1802 & Unspecified & [180] \\
\hline Melanosuchus niger (Spix, 1825) - Black cayman & $\begin{array}{l}\text { Thrombosis, infection, } \\
\text { swelling, asthma, amulet } \\
\text { used as a protection } \\
\text { against snake bite, injuries } \\
\text { caused by spines of the } \\
\text { 'arraia', pain relief in injuries } \\
\text { caused by snake bites }\end{array}$ & {$[7-9,62,139]$} \\
\hline Paleosuchus palpebrosus (Cuvier, 1807) - Cayman & $\begin{array}{l}\text { Snake bite, asthma, stroke, } \\
\text { rheumatism, thrombosis, } \\
\text { backache, sexual } \\
\text { impotence, edema, } \\
\text { mycosis, evil eye, irritation } \\
\text { when milk teeth are } \\
\text { erupting, discharge, sore } \\
\text { throat, amulet used as a } \\
\text { protection against snake } \\
\text { bite, hernia, prostate } \\
\text { problems }\end{array}$ & {$[7-9,62,71,139]$} \\
\hline Paleosuchus trigonatus (Schneider, 1801) & Rheumatism & {$[62,69]$} \\
\hline \multicolumn{3}{|l|}{ Crocodylidae } \\
\hline Crocodylus moreletii Duméril \& Bibron 1851 & Asthma, bronchial diseases & [93] \\
\hline Crocodylus acutus Cuvier 1807 & Unspecified & [152] \\
\hline
\end{tabular}


Table 1 Medicinal animals and its respective uses in popular medicine in Latin America (Continued)

\begin{tabular}{|c|c|c|}
\hline BIRDS & & \\
\hline Turdidae & & \\
\hline Turdus chiguanco Lafresnaye \& d'Orbigny, 1837 & Stomach ache & [189] \\
\hline Turdus grayi Bonaparte, 1838 & Unspecified & [152] \\
\hline Troglodytes musculus Naumann, 1823 & Epilepsy & [72] \\
\hline Cariamidae & & \\
\hline Cariama cristata (Linnaeus, 1766) & Ethnoveterinary use & {$[127,178]$} \\
\hline Accipitridae & & \\
\hline $\begin{array}{l}\text { Geranoaetus melanoleucus (Vieillot, 1819) - Black-chested Buzzard- } \\
\text { Eagle }\end{array}$ & $\begin{array}{l}\text { The feathers are used as } \\
\text { inensing and to } \\
\text { makemasks, wrinkles. }\end{array}$ & [148] \\
\hline Harpia harpyja (Linnaeus, 1758) & Unspecified & [179] \\
\hline Buteogallus urubitinga (J. F. Gmelin, 1788) & $\begin{array}{l}\text { Backache, column pain and } \\
\text { rheumatism }\end{array}$ & [66] \\
\hline Spizastur melanoleucus (Vieillot, 1816) & Unspecified & [152] \\
\hline Parabuteo unicinctus (Temminck, 1824) & Snake bite & [152] \\
\hline Anatidae & & \\
\hline Anser anser (Linnaeus, 1758) - Greylag goose & $\begin{array}{l}\text { Laryngitis, pharyngitis, } \\
\text { tonsillitis }\end{array}$ & [97] \\
\hline Anas platyrhynchos Linnaeus, 1758 - mallard & $\begin{array}{l}\text { General weaknesses, sexual } \\
\text { weakness, nervous } \\
\text { disturbances }\end{array}$ & [66] \\
\hline Netta erythrophthalma (Wied-Neuwied, 1833)- 'paturi' & $\begin{array}{l}\text { Male impotence and } \\
\text { weakness }\end{array}$ & [66] \\
\hline Cairina moschata (Linnaeus, 1758) & Unspecified & [111] \\
\hline Lophodytes cucullatus (Linnaeus, 1758) & Unspecified & [96] \\
\hline Tytonidae & & \\
\hline Tyto alba (Scopoli, 1769) & Unspecified & [152] \\
\hline Anhimidae & & \\
\hline Anhima cornuta (Linnaeus, 1766) - Horned screamer, anuhma & $\begin{array}{l}\text { Intoxication from } \\
\text { poisonous animals }\end{array}$ & [151] \\
\hline Scolopacidae & & \\
\hline Actitis macularius (Linnaeus, 1766) & Dandruff (seborrhea) & [96] \\
\hline Ardeidae & & \\
\hline Ardea cocoi (Linnaeus, 1766) - White-necked Heron & $\begin{array}{l}\text { Swelling, inflammation, } \\
\text { injuries caused by the } \\
\text { spines of the 'arraia' and } \\
\text { others fishes, asthma, boil, } \\
\text { tumour, rheumatism, } \\
\text { earache }\end{array}$ & {$[7-9,87]$} \\
\hline Ardea alba Linnaeus, 1758 & $\begin{array}{l}\text { Bronquithis and } \\
\text { pneumonia }\end{array}$ & [148] \\
\hline Nycticorax nycticorax (Linnaeus, 1758) & Unspecified & [96] \\
\hline Apodidae & & \\
\hline Streptoprocne zonaris (Shaw, 1796) & Unspecified & [106] \\
\hline Casmerodius albus (Gmelin, 1789) - Garça & $\begin{array}{l}\text { Bronquithis and } \\
\text { pneumonia }\end{array}$ & [71] \\
\hline Ardeidae & & \\
\hline Tigrisoma lineatum (Boddaert, 1783) - socó, Rufescent Tiger-Heron & $\begin{array}{l}\text { Bronquithis and } \\
\text { pneumonia }\end{array}$ & [71] \\
\hline Caprimulgidae & & \\
\hline Nyctidromus albicollis (Gmelin, 1789) - Pauraque & Amulets, snake bite & [69] \\
\hline Caprimulgus vociferus A. Wilson, 1812 & Unspecified & [38] \\
\hline
\end{tabular}


Table 1 Medicinal animals and its respective uses in popular medicine in Latin America (Continued)

\section{Cathartidae \\ Coragyps atratus (Bechstein, 1793) - Black vulture}

Deafness, bronchitis, anaemia, alcoholism, asthma, flu, earache, rheumatism, pain in the parturition, mal aire, swelling, epilepsy

Cathartes aura (Linnaeus, 1758) - Urubu, urubu-de-cabeçavermelha

\section{Cracidae}

Penelope jacucaca (Spix, 1825) - White-browed guan

Penelope superciliaris Temminck, 1815

Penelope purpurascens Wagler, 1830

Penelope jacquacu Spix, 1825

Crax globulosa Spix, 1825 - Wattled Curassow

Crax rubra Linnaeus, 1758

Aburria pipile (Jacquin, 1784)

Pauxi tuberosa (Spix, 1825)- Razor-billed Curassow

\section{Unspecified}

$[4,91,103]$
$[7-9,31,42,97]$

\begin{tabular}{l} 
Mitu tuberosum (Spix, 1825) \\
\hline Ortalis guttata (Spix, 1825) \\
\hline Ortalis vetula (Wagler, 1830)
\end{tabular}

\section{Psophiidae}

\begin{tabular}{|c|c|c|}
\hline Psophia leucoptera Spix, 1825 & Unspecified & {$[179]$} \\
\hline \multicolumn{3}{|l|}{ Phoenicopteridae } \\
\hline Phoenicopterus andinus Philippi, 1854 & $\begin{array}{l}\text { To alliviate labor pain, } \\
\text { sprains and distend }\end{array}$ & [148] \\
\hline Phoenicopterus chilensis Molina, 1782 & $\begin{array}{l}\text { To alliviate labor pain, } \\
\text { sprains and distend }\end{array}$ & [148] \\
\hline Phoenicopterus jamesi Sclater, 1886 & $\begin{array}{l}\text { To alliviate labor pain, } \\
\text { sprains and distend }\end{array}$ & [148] \\
\hline \multicolumn{3}{|l|}{ Ciconiidae } \\
\hline Vultur gryphus Linnaeus, 1758 & $\begin{array}{l}\text { Stomach ache, poor } \\
\text { digestion, boils }\end{array}$ & [189] \\
\hline Ciconia maguari (Gmelin, 1789) - Maguari stork & $\begin{array}{l}\text { Injuries caused by the } \\
\text { spines of the 'arraia' and } \\
\text { others fishes, thrombosis }\end{array}$ & {$[7-9,82]$} \\
\hline Jabiru mycteria (Lichtenstein, 1819) - Jabiru & Unspecified & [82] \\
\hline Sarcoramphus papa (Linnaeus, 1758) - King Vulture & Epilepsia, sifilis, ulcera & [96] \\
\hline \multicolumn{3}{|l|}{ Columbidae } \\
\hline Leptotila rufaxilla (Richard \& Bernard, 1792) - Gray-fronted dove & Thrombosis, pterygium & {$[66,162]$} \\
\hline Columba livia (Gmelin, 1789) - Rock pigeon & $\begin{array}{l}\text { Asthma, laryngitis, } \\
\text { pharyngitis, tonsilitis }\end{array}$ & {$[66,97,103]$} \\
\hline Columba picazuro Temminck, 1813 - 'asa-branca & $\begin{array}{l}\text { Sore throat, tonsillitis, } \\
\text { bronchitis and hoarseness }\end{array}$ & [66] \\
\hline Columbina talpacoti (Temminck, 1810) & Unspecified & {$[175]$} \\
\hline Columbina passerina (Linnaeus, 1758) & Unspecified & [96] \\
\hline \multicolumn{3}{|l|}{ Corvidae } \\
\hline Cyanocorax cyanopogon (Wied, 1821) - White-naped jay & $\begin{array}{l}\text { Asthma, neurological } \\
\text { problems }\end{array}$ & {$[66,81,162]$} \\
\hline \multicolumn{3}{|l|}{ Cotingidae } \\
\hline Procnias nudicollis (Vieillot, 1817) - araponga, Bare-throated Bellbird & Tuberculosis & {$[4,71]$} \\
\hline
\end{tabular}


Table 1 Medicinal animals and its respective uses in popular medicine in Latin America (Continued)

\begin{tabular}{|c|c|c|}
\hline Cotinga amabilis Gould, 1857 & Unspecified & [96] \\
\hline Crotophaga ani Linnaeus, 1758 - Smooth-billed ani & $\begin{array}{l}\text { Bronchitis, thrombosis, } \\
\text { asthma, whooping cough, } \\
\text { rheumatism }\end{array}$ & {$[7-9,97]$} \\
\hline Crotophaga sulcirostris Swainson, 1827 & Cancer & {$[105]$} \\
\hline Piaya cayana (Linnaeus, 1766) & Heart diseases & [105] \\
\hline Guira guira (Gmelin, 1788) - Guira cuckoo & Asthma & {$[97]$} \\
\hline Geococcyx velox (Wagner, 1836) & Unspecified & [91] \\
\hline Geococcyx californianus (Lesson, 1829) & Cancer & [103] \\
\hline \multicolumn{3}{|l|}{ Charadriidae } \\
\hline Vanellus chilensis (Molina, 1782) - Southern lapwing & To stay awake & {$[4,115,162]$} \\
\hline \multicolumn{3}{|l|}{ Emberezidae } \\
\hline Coereba flaveola (Linnaeus, 1758)- Banana quit & Thrombosis & [162] \\
\hline \multicolumn{3}{|l|}{ Numididae } \\
\hline Numida meleagris Linnaeus, 1758 - Helmeted Guineafowl, "Guiné" & Whooping cough & {$[4]$} \\
\hline \multicolumn{3}{|l|}{ Falconidae } \\
\hline Herpetotheres cachinnans (Linnaeus, 1758) - Laughing falcon & $\begin{array}{l}\text { Snake bite, sore throat, } \\
\text { tonsillitis and hoarseness }\end{array}$ & {$[66,69]$} \\
\hline Caracara plancus (Miller, 1777) - Southern caracara & Snake bite & [69] \\
\hline Falco rufigularis Daudin, 1800 - Bat falcon & Snake bite & {$[69,82]$} \\
\hline \multicolumn{3}{|l|}{ Opisthocomidae } \\
\hline Opisthocomus hoazin (Statius Muller, 1776) & Unspecified & {$[82]$} \\
\hline \multicolumn{3}{|l|}{ Furnaridae } \\
\hline Furnarius rufus (Gmelin, 1788)- Rufous hornero & Mumps & {$[4,114]$} \\
\hline \multicolumn{3}{|l|}{ Meleagrididae } \\
\hline Meleagris gallopavo Linnaeus, 1758 - turkey & Asthma & {$[7,9]$} \\
\hline \multicolumn{3}{|l|}{ Odontophoridae } \\
\hline Odontophorus capueira (Spix, 1825) & Unspecified & [111] \\
\hline Callipepla squamata (Vigors, 1830) & Unspecified & [96] \\
\hline Cyrtonyx montezumae (Vigors, 1830) & Evil eye & [103] \\
\hline Colinus virginianus (Linnaeus, 1758) & Dandruff, fever & [96] \\
\hline \multicolumn{3}{|l|}{ Phasianidae } \\
\hline Gallus gallus (Linnaeus, 1758) - Domestic chicken & $\begin{array}{l}\text { Catarrh, fever, warts, } \\
\text { haemorrhage, bronchitis, } \\
\text { nasal congestion, flu, skin } \\
\text { thorns and wounds, } \\
\text { asthma, sore throat, } \\
\text { tumour, poor digestion, } \\
\text { healing of umbilical cord } \\
\text { of newborn baby, swelling, } \\
\text { cough, tuberculosis, } \\
\text { earache, tonsillitis, } \\
\text { rheumatism, diarrhoea, } \\
\text { inflammation, pneumonia, } \\
\text { Renal calculus, headache, } \\
\text { sore throat, nasal } \\
\text { congestion, fever, swelling }\end{array}$ & {$[7-9,31,42,63,68,81,85,97,103,109,110,115,162,164,166]$} \\
\hline Pavo cristatus Linnaeus, 1758 - Indian peafowl & $\begin{array}{l}\text { Thrombosis, epilepsy, } \\
\text { fatigue }\end{array}$ & {$[30,126]$} \\
\hline Coturnix coturnix (Linnaeus, 1758)-'codorna & $\begin{array}{l}\text { Male impotence, urinary } \\
\text { infection and weakness }\end{array}$ & {$[66]$} \\
\hline \multicolumn{3}{|l|}{ Picidae } \\
\hline Dryocopus lineatus (Linnaeus, 1766) - Lineated woodpecker & Sexual impotence & [69] \\
\hline Colaptes rupicola Orbigny, 1840 & Unspecified & [189] \\
\hline Melanerpes formicivorus (Swainson, 1827) & Epilepsy & {$[105]$} \\
\hline Melanerpes aurifrons (Wagler, 1829) & Headache & {$[96]$} \\
\hline
\end{tabular}


Table 1 Medicinal animals and its respective uses in popular medicine in Latin America (Continued)

\begin{tabular}{|c|c|c|}
\hline Psittacidae & & \\
\hline Amazona aestiva (Linnaeus, 1758) & Asthma & {$[7,9]$} \\
\hline Ara ambiguus (Bechstein, 1811) & Unspecified & [152] \\
\hline Amazona farinosa (Boddaert, 1783) & Unspecified & [152] \\
\hline Amazona autumnalis (Linnaeus, 1758) & Unspecified & [152] \\
\hline Amazona auropalliata (Lesson, 1842) & Unspecified & [152] \\
\hline Amazona albifrons (Sparrman, 1788) & Unspecified & {$[152]$} \\
\hline Pionus senilis (Spix, 1824) & Unspecified & [152] \\
\hline Pionus menstruus (Linnaeus, 1766) & Unspecified & [152] \\
\hline Pionopsitta haematotis (P. L. Sclater \& Salvin, 1860) & Unspecified & [152] \\
\hline Ara macao (Linnaeus, 1758) & Mental problems & [152] \\
\hline \multicolumn{3}{|l|}{ Hirundinidae } \\
\hline Petrochelidon andecola (D’Orbigny \& Lafresnaye, 1837) & Epilepsy, heart diseases & {$[189]$} \\
\hline Stelgidopteryx ruficollis (Vieillot, 1817) & Cyst & {$[175]$} \\
\hline Hirundo rustica Linnaeus, 1758 - Barn Swallow & $\begin{array}{l}\text { Dandruff, muscular pain, } \\
\text { ophthalmological } \\
\text { problems, Insomnia, }\end{array}$ & [96] \\
\hline \multicolumn{3}{|l|}{ Strigidae } \\
\hline Glaucidium brasilianum (Gmelin, 1788) & Rheumatism & [152] \\
\hline \multicolumn{3}{|l|}{ Alcedinidae } \\
\hline Chloroceryle americana (Gmelin, 1788) & Unspecified & [152] \\
\hline \multicolumn{3}{|l|}{ Cardinalidae } \\
\hline Passerina versicolor (Bonaparte, 1838) & Unspecified & [96] \\
\hline \multicolumn{3}{|l|}{ Rallidae } \\
\hline Aramides cajanea (Statius Muller, 1776) - Grey-necked wood-rail & Evil eye & [162] \\
\hline \multicolumn{3}{|l|}{ Struthionidae } \\
\hline Struthio camelus Linnaeus, 1766 - Common Ostrich & Osteoporosis & [136] \\
\hline \multicolumn{3}{|l|}{ Ramphastidae } \\
\hline Ramphastos tucanus Linnaeus, 1758 - Red-billed Toucan & $\begin{array}{l}\text { Thrombosis, sexual } \\
\text { impotence }\end{array}$ & {$[31,69]$} \\
\hline Ramphastos vitellinus Lichtenstein, 1823 - Channel-billed toucan & Sexual impotence & [69] \\
\hline Ramphastos sulfuratus Lesson, 1830 & Unspecified & [77] \\
\hline Pteroglossus aracari (Linnaeus, 1758) - Black-necked aracari & Sexual impotence & [69] \\
\hline Pteroglossus inscriptus - Swainson, 1822, Lettered aracari & Sexual impotence & [69] \\
\hline Pteroglossus torquatus (Gmelin, 1788) & Unspecified & [152] \\
\hline Pteroglossus frantzii Cabanis, 1861 & Unspecified & [152] \\
\hline Selenidera spectabilis Cassin, 1858 & Unspecified & [152] \\
\hline \multicolumn{3}{|l|}{ Rheidae } \\
\hline Rhea americana (Linnaeus, 1758) - Greater rhea & $\begin{array}{l}\text { General aches, rheumatism, } \\
\text { thrombosis, strokes }\end{array}$ & {$[63,115,162,164,166]$} \\
\hline Pterocnemia pennata (Orbigny, 1834) & Unspecified & [148] \\
\hline \multicolumn{3}{|l|}{ Trogonidae } \\
\hline Pharomachrus mocinno De la Llave, 1832 & Unspecified & [152] \\
\hline \multicolumn{3}{|l|}{ Tinamidae } \\
\hline Crypturellus noctivagus (Wied, 1820) - Yellow-legged tinamou & $\begin{array}{l}\text { Thrombosis, stroke, snake } \\
\text { bites, tuberculosis, deafness }\end{array}$ & {$[71,115,162]$} \\
\hline Crypturellus variegatus (Gmelin, 1789) - Inambu-galinha & Unspecified & [87] \\
\hline Nothura boraquira (Spix, 1825) - White-bellied nothura & $\begin{array}{l}\text { Thrombosis, stroke, } \\
\text { tootache }\end{array}$ & {$[115,162]$} \\
\hline Nothura maculosa cearensis Naumburg, 1932 - Spotted Nothura & Effusion, snake bite & {$[178]$} \\
\hline Rhynchotus rufescens (Temminck, 1815) - Red-winged tinamou & $\begin{array}{l}\text { Snake bite, thrombosis, } \\
\text { tuberculosis }\end{array}$ & {$[119,162]$} \\
\hline
\end{tabular}


Table 1 Medicinal animals and its respective uses in popular medicine in Latin America (Continued)

\begin{tabular}{|c|c|c|}
\hline Tinamus solitarius (Viellot 1819) - Macuco, macuca & Unspecified & [111] \\
\hline \multicolumn{3}{|l|}{ Trochilidae } \\
\hline $\begin{array}{l}\text { Eupetomena macroura (Gmelin, 1788) - Swallow-tailed } \\
\text { hummingbird }\end{array}$ & $\begin{array}{l}\text { Cardiopathies, asthma, flu, } \\
\text { pains }\end{array}$ & [97] \\
\hline Lesbia nuna (Lesson, 1832) & Evil eye & [189] \\
\hline \multicolumn{3}{|l|}{ Tyrannidae } \\
\hline Fluvicola nengeta (Linnaeus, 1766) - Masked water-tyrant & Boils, asthma & [66] \\
\hline Pitangus sulphuratus (Linnaeus, 1766) & Earache & [97] \\
\hline \multicolumn{3}{|l|}{ Icteridae } \\
\hline Quiscalus mexicanus (Gmelin, 1788) & Unspecified & [38] \\
\hline Psarocolius wagleri (G. R. Gray, 1845) & Unspecified & [152] \\
\hline \multicolumn{3}{|l|}{ Emberizidae } \\
\hline Zonotrichia capensis (Statius Muller, 1776) & Unspecified & [189] \\
\hline \multicolumn{3}{|l|}{ MAMMALS } \\
\hline \multicolumn{3}{|l|}{ Molossidae } \\
\hline Molossus molossus (Pallas, 1766), Pallas' free-tailed bat (Bat) & Asthma & {$[66,176]$} \\
\hline \multicolumn{3}{|l|}{ Noctilionidae } \\
\hline Noctilio leporinus (Linnaeus, 1758) - greater bulldog bat & Unspecified & [152] \\
\hline \multicolumn{3}{|l|}{ Phyllostomidae } \\
\hline Artibeus jamaicensis Leach, 1821 - bat & Cyst & [175] \\
\hline \multicolumn{3}{|l|}{ Agoutidae } \\
\hline Agouti paca (Linnaeus, 1766) - Spotted paca & $\begin{array}{l}\text { Wound in the breast } \\
\text { caused by suckling, } \\
\text { ophthalmological } \\
\text { problems, stomach } \\
\text { disorders, pterygium, to } \\
\text { suck a splinter out of skin } \\
\text { or flesh, injuries caused by } \\
\text { the spines of 'arraia', } \\
\text { control cholesterol level, } \\
\text { thrombosis, general body } \\
\text { pain, leishmaniasis, snake } \\
\text { bite, rheumatism, heart } \\
\text { pain, pain in bones, liver } \\
\text { pain, fever, child birth, ant } \\
\text { bite }\end{array}$ & {$[7-9,31,70,85,97,162]$} \\
\hline \multicolumn{3}{|l|}{ Balaenopteridae } \\
\hline Megaptera novaeangliae Borowski, 1781 & Unspecified & {$[80,171]$} \\
\hline Balaenoptera acutorostrata Lacépède, 1804 - Minke whale & $\begin{array}{l}\text { Rheumatism, sore throat, } \\
\text { wounds }\end{array}$ & [7-9] \\
\hline \multicolumn{3}{|l|}{ Bovidae } \\
\hline Bos taurus Linnaeus, 1758 - Cow & $\begin{array}{l}\text { Thrombosis, evil eye, } \\
\text { amulet used as a } \\
\text { protection against snake } \\
\text { bite, baldness, sexual } \\
\text { impotence, measles, } \\
\text { varicella, anaemia, } \\
\text { whooping cough, } \\
\text { Alcoholism, rheumatism, } \\
\text { inflammation, asthma, } \\
\text { cough, sore throat, } \\
\text { wounds, cracks in the sole } \\
\text { of the feet, bronchitis, } \\
\text { dizziness, bladder } \\
\text { problems, varicella, removal } \\
\text { of thorns, paralisia facial, } \\
\text { nervousness, earache, } \\
\text { migraine, }\end{array}$ & {$[7-9,31,36,42,63,115,162-164,166]$} \\
\hline Bubalus bubalis (Linnaeus, 1758) - Water buffalo (feral) & $\begin{array}{l}\text { Rheumatism, osteoporosis, } \\
\text { thrombosis }\end{array}$ & [8] \\
\hline
\end{tabular}


Table 1 Medicinal animals and its respective uses in popular medicine in Latin America (Continued)

Ovis aries (Linnaeus, 1758) - Sheep

Edema, fractures, erysipelas, $\quad[7-9,31,36,42,63,91,97,115,162-164,166]$

herpes zoster, backache,

swelling, to assist children

who take longer than

usual to start walking,

arthritis, arthrosis,

rheumatism, muscle strain,

inflammation, luxation,

cracks in the sole of the

feet, joint pain, removal of

thorns

Capra hircus Linnaeus, 1758 - Domestic goat

Evil eye, snake bite, muscle $\quad[7-9,31,42,91]$

strain

\section{Geomyidae}

Orthogeomys hispidus (LeConte, 1852)

Stomach ache, fever, susto $\quad[38,40]$

and espanto, swelling,

madness

\section{Bradypodidae}

Bradypus variegatus Shinz, 1825 - Brown-throated three-toed sloth

Bradypus tridactylus Linnaeus, 1758 - Pale-throated three-toed

sloth

$\begin{array}{ll}\text { Thrombosis } & {[8,30,97]} \\ \begin{array}{l}\text { Thrombosis, insects bite, } \\ \text { scorpions bite }\end{array} & {[70]}\end{array}$

\section{Canidae}

Lycalopex culpaeus (Molina, 1782)

Scare, susto" (fright)

[154]

Lycalopex gymnocercus (G. Fischer, 1814)

Air loss, asthma, backache,

disorders after parturition,

pain in bones, rheumatism,

scare, sprains, ulcer

Canis lupus (Linnaeus, 1758) - Domestic dog

Chicken pox, mumps,

smallpox, asthma, varicella,

measles, menstrual cramps

Canis latrans Say, 1823

Rheumatism

$[100,154]$

Cerdocyon thous (Linnaeus, 1766) - Crab-eating fox

Rheumatism, flu

haemorrhoids, disorders

after parturition, diabetes,

thrombosis, backache,

osteoporosis, eczema, pain

in articulations, sore throat,

womb inflammation

Chrysocyon brachyurus (Illiger, 1815) - Maned wolf

Epilepsy

$[7-9,31,97,115,162]$

Dusicyon thous - Linnaeus, 1766 - Crab-eating fox

Alcoholism, thrombosis,

$[133,161,162]$

rheumatism,

$[97,133]$

ophthalmological

problems, diabetes, urinary

infection

\begin{tabular}{lll}
\hline Speothos venaticus (Lund, 1842) - Bush dog & Haemorrhoids & {$[97,133]$} \\
\hline Caviidae & & \\
\hline Galea spixii Wagler, 1831 & Unspecified & {$[80]$} \\
\hline Cavia aperea Erxleben, 1777 & Inflammation, teething & {$[97]$} \\
\hline Cavia porcellus (Linnaeus, 1758) & nervios encogidos. & {$[182]$} \\
\hline Kerodon rupestris (Wied-Neuwied, 1820) & $\begin{array}{l}\text { Constipation, tired sight, } \\
\text { effusion }\end{array}$ & {$[162]$} \\
\hline Cebidae & & \\
\hline Alouatta belzebul (Linnaeus, 1766) - Red-handed howler monkey & $\begin{array}{l}\text { Whooping cough, sore } \\
\text { throat, asthma }\end{array}$ & {$[7-9,137]$} \\
\hline Alouatta nigerrima Lönnberg, 1941 - Amazon black howler & $\begin{array}{l}\text { Whooping cough, } \\
\text { inflammation }\end{array}$ & {$[69,137,181]$} \\
\hline
\end{tabular}


Table 1 Medicinal animals and its respective uses in popular medicine in Latin America (Continued)

\begin{tabular}{|c|c|c|}
\hline Alouatta seniculus (Linnaeus, 1766) - Red howler monkey & $\begin{array}{l}\text { Whooping cough, } \\
\text { inflammation, to accelerate } \\
\text { parturition }\end{array}$ & {$[69,87,137]$} \\
\hline Allouatta fusca (É. Geoffroy, 1812) - Bugio & $\begin{array}{l}\text { Used as aphrodisiac; used } \\
\text { to treat any disease }\end{array}$ & {$[101,111]$} \\
\hline Alouatta sara Elliot, 1910 & Unspecified & {$[83,137]$} \\
\hline Alouatta palliata (Gray 1849) & $\begin{array}{l}\text { General pains and } \\
\text { inflammation }\end{array}$ & {$[107,137]$} \\
\hline Ateles chamek (Humboldt, 1812) & $\begin{array}{l}\text { Fever, cough, cold } \\
\text { shoulder pain, sleeping } \\
\text { problems, leishmaniosis, } \\
\text { spider bite, snake bites. }\end{array}$ & {$[83,137]$} \\
\hline Ateles geoffroyi Kuhl, 1820 & Rheumatism & {$[137,152]$} \\
\hline Ateles paniscus (Linnaeus 1758) & Rheumatism & [137] \\
\hline Aotus azarai (Humboldt, 1811) & To cure dribbling in babies & {$[137]$} \\
\hline Aotus griseimembra Elliot 1912 & Unspecified & {$[137]$} \\
\hline Cebus apella (Linnaeus, 1758) - Brow capuchin & $\begin{array}{l}\text { Insect sting, eye infection, } \\
\text { Inflammatory processes, } \\
\text { insect sting, used for } \\
\text { osteomuscular problems, } \\
\text { eye infection and male } \\
\text { impotency }\end{array}$ & {$[7-9,42,137]$} \\
\hline Cebus albifrons (Humboldt 1812 & Used as fortifier & {$[95,137,152]$} \\
\hline Cebus capucinus (Linnaeus 1758) & Unspecified & {$[137]$} \\
\hline Lagothrix lagotricha (Humboldt 1812) & Unspecified & {$[95,137]$} \\
\hline \multicolumn{3}{|l|}{ Callitrichidae } \\
\hline Saguinus fuscicollis (Spix, 1823) & Impotence & {$[83]$} \\
\hline Saguinus mystax (Spix 1823) & Impotence & {$[83]$} \\
\hline \multicolumn{3}{|l|}{ Cervidae } \\
\hline Blastocerus dichotomus (Illiger, 1815) - Marsh deer & Diarrhoea, vomit & {$[151]$} \\
\hline Mazama americana (Erxleben, 1777) - Red brocket & Stroke, cold, burns & {$[7-9,30,42,115]$} \\
\hline Mazama simplicicornis (Illinger, 1811) & $\begin{array}{l}\text { Diarrhoea, verminosis, evil } \\
\text { eye }\end{array}$ & {$[69]$} \\
\hline Mazama cf. gouazoupira (G. Fischer, 1814) - Gray brocket & $\begin{array}{l}\text { Asthma, edema, } \\
\text { rheumatism, snake bite, } \\
\text { thrombosis, to assit } \\
\text { children who take longer } \\
\text { than usual to start walking, } \\
\text { tootache, wounds, sprains }\end{array}$ & {$[8,63,97,162,164]$} \\
\hline Ozotocerus bezoarticus (Linnaeus, 1758) & $\begin{array}{l}\text { Diarrhoea, verminosis, evil } \\
\text { eye }\end{array}$ & [69]) \\
\hline Odocoileus virginianus (Zimmermann, 1780) & $\begin{array}{l}\text { heart diseases, } \\
\text { oftalmological problems, } \\
\text { Didelphis virginiana }\end{array}$ & {$[91,103]$} \\
\hline \multicolumn{3}{|l|}{ Dasypodidae } \\
\hline Dasypus novemcinctus (Linnaeus, 1758) - Nine-banded armadillo & $\begin{array}{l}\text { Thrombosis, insects bite, } \\
\text { scorpions bite, edema, } \\
\text { asthma, deafness, earache, } \\
\text { evil eye, diarrhoea, } \\
\text { whooping cough, } \\
\text { tuberculosis, to accelerate } \\
\text { parturition }\end{array}$ & {$[7,8,31,42,97,162]$} \\
\hline Dasypus kappleri Krauss, 1862 & Earache & {$[84]$} \\
\hline Euphractus sexcinctus (Linnaeus, 1758) - Six-banded armadillo & $\begin{array}{l}\text { Wounds, earache, evil eye, } \\
\text { asthma, sore throat, } \\
\text { pneumonia, sinusitis, } \\
\text { deafness, coarse throat }\end{array}$ & {$[7,8,31,42,97,162]$} \\
\hline
\end{tabular}


Table 1 Medicinal animals and its respective uses in popular medicine in Latin America (Continued)

\begin{tabular}{|c|c|c|}
\hline $\begin{array}{l}\text { Tolypeutes tricinctus (Linnaeus, 1758) - Brazilian three-banded } \\
\text { armadillo }\end{array}$ & Thrombosis, rheumatism & {$[151,162]$} \\
\hline Priodontes maximus (Kerr, 1792) & $\begin{array}{l}\text { Embolism, ant bite, visions, } \\
\text { skin diseases }\end{array}$ & [148] \\
\hline Chaetophractus vellerosus (Gray, 1865) & General diseases & [148] \\
\hline Cabassous unicinctus (Linnaeus, 1758) - Tatu-rabo-de-couro & Unspecified & {$[88,90]$} \\
\hline Cabassous centralis (Miller, 1899) - Northern Naked-tailed Ar & Stomach ache & {$[104,152]$} \\
\hline
\end{tabular}

\section{Dasyproctidae}

\begin{tabular}{lll}
\hline Dasyprocta prymnolopha Wagler, 1831 - Black-rumped agouti & Asthma, thrombosis & {$[4,162]$} \\
\hline Dasyprocta variegata - Brown agouti & $\begin{array}{l}\text { Childbirth, Impotence, } \\
\text { snake bite }\end{array}$ & {$[148]$}
\end{tabular}

\section{Delphinidae}

Sotalia fluviatilis Gervais \& Deville, 1853) - Gray dolphin, gray river dolphin

Asthma, headache,

$[7-9,31,42,143]$

rheumatism, hernia, womb

disorders, sore throat,

injuries caused by the

spines of the 'arraia',

swelling, haemorrhoids

inflammation, wounds,

earache, erysipelas,

athlete's foot, tumour, cancer

Sotalia guianensis (P. J. Van Bénéden, 1864) - Guianan river Asthma, headache,

rheumatism, hernia, womb

disorders, sore throat,

injuries caused by the spines of the 'arraia', swelling, haemorrhoids inflammation, wounds, earache, erysipelas,

athlete's foot, tumour, cancer

\section{Didelphidae}

Didelphis albiventris (Lund, 1840) - Common opossum Boils, rheumatism

[8]

Didelphis marsupialis (Linnaeus, 1758) - Southern opossum

Acne, wounds, bronchitis, joint pain, stomach ache, rheumatism, diarrhoea, inflammation, erysipelas, pain in gestation, asthma headache, oothache, earache, sore throat, flu, fever, body pain, fatigue, to accelerate parturition, mal aire, swelling

Didelphis virginiana Kerr, 1792

Rheumatism, skin spots, $\quad[91,103]$

acne, anaemia, to

accelerate parturition, mal aire, swelling

\begin{tabular}{lll}
\hline Didelphis aurita (Wied-Neuwied, 1826) - Saruê & Unspecified & {$[88,90]$} \\
\hline Philander opossum (Linnaeus, 1758) - Gray Four-eyed Opossum & Unspecified & {$[152]$} \\
\hline Megalonychidae & & \\
\hline Choloepus hoffmanni Peters, 1858 & $\begin{array}{l}\text { Visions, hallucination, } \\
\text { cramps }\end{array}$ & {$[148]$} \\
\hline Erethizontidae & &
\end{tabular}

Coendou bicolor (Tschudi, 1844)

Hallucination, fever, ant bite, flu, whooping cough, scare, varicose veins

Coendou prehensilis (Linnaeus, 1758) - Brazilian porcupine
Bronchitis, thrombosis, epilepsy, stroke, abscesses, conjunctivitis, asthma

\section{[30]}

$[7-9,36,42,63,73,97,115,161,162,164]$ 
Table 1 Medicinal animals and its respective uses in popular medicine in Latin America (Continued)

\begin{tabular}{|c|c|c|}
\hline Coendou villosus (Cuvier, 1822) - Ouriço-cacheiro & Unspecified & [111] \\
\hline Sphiggurus mexicanus (Kerr, 1792) & Acne, wart & [91] \\
\hline Sphiggurus insidiosus (Lichtenstein, 1818) - Luís-cacheiro & Unspecified & [89] \\
\hline Chaetomys subspinosus(Olfers, 1818) - Luís-cacheiro & Unspecified & [89] \\
\hline \multicolumn{3}{|l|}{ Equidae } \\
\hline Equus asinus Linnaeus, 1758 - Asino & $\begin{array}{l}\text { Snake bite, whooping } \\
\text { cough, asthma, Avoid } \\
\text { pregnancy }\end{array}$ & {$[7-9,42,73,91,115,162]$} \\
\hline Equus caballus (Linnaeus, 1758) - Horse & $\begin{array}{l}\text { Cough, deep cuts, } \\
\text { dermatosis, wounds }\end{array}$ & [8] \\
\hline \multicolumn{3}{|l|}{ Felidae } \\
\hline Felis silvestris Schreber, 1775 - Domestic cat & Asthma, snake bites & [8] \\
\hline Puma concolor (Linnaeus, 1771) - Mountain lion & $\begin{array}{l}\text { Wounds, leishmaniosis, } \\
\text { arthritis, pain in bones, } \\
\text { rheumatism, distend, scare, } \\
\text { stomach ache, evil eye, } \\
\text { fever, avoid acne, } \\
\text { contusions and muscular } \\
\text { pain }\end{array}$ & {$[8,87,157]$} \\
\hline Panthera onca (Linnaeus, 1758) & $\begin{array}{l}\text { Wounds, leishmaniosis, } \\
\text { Cough, fatigue, fever, pain } \\
\text { in bones }\end{array}$ & {$[8,87,157]$} \\
\hline Panthera tigris (Linnaeus, 1758) - Tigre & Unspecified & [4] \\
\hline Leopardus jacobitus (Cornalia, 1865) & Self encorage & [148] \\
\hline Leopardus colocolo (Molina, 1782) & Self encorage & [148] \\
\hline Leopardus wiedii (Schinz, 1821) & Unspecified & [148] \\
\hline Leopardus pardalis (Linnaeus, 1758) - Gato-maracajá & Unspecified & [30] \\
\hline \multicolumn{3}{|l|}{ Octodontidae } \\
\hline Ctenomys opimus Wagner, 1848 & $\begin{array}{l}\text { To make the child's teeth } \\
\text { stronger }\end{array}$ & [148] \\
\hline \multicolumn{3}{|l|}{ Chinchillidae } \\
\hline Lagidium viscacia (Molina, 1782) & Bad memory & [148] \\
\hline Lagidium peruanum Meyen, 1833 & Earache & {$[182,189]$} \\
\hline \multicolumn{3}{|l|}{ Hydrochaeridae } \\
\hline Hydrochaeris hydrochaeris (Linnaeus, 1766) - Capybara & $\begin{array}{l}\text { Thrombosis, conjunctivitis, } \\
\text { venereal disease, } \\
\text { rheumatism, earache, } \\
\text { strengthen bones, liver } \\
\text { pain, bronchitis, asthma, } \\
\text { wounds, erysipelas, cough }\end{array}$ & {$[7-9,42,73,85,97,115,155,161,162]$} \\
\hline \multicolumn{3}{|l|}{ Heteromyidae } \\
\hline Heteromys desmarestianus Gray, 1868 & Unspecified & {$[38,148]$} \\
\hline \multicolumn{3}{|l|}{ Iniidae } \\
\hline Inia geoffrensis (Blainville, 1817) - Amazon river dolphin & $\begin{array}{l}\text { Asthma, headache, } \\
\text { rheumatism, hernia, womb } \\
\text { disorders, sore throat, } \\
\text { injuries caused by the } \\
\text { spines of the 'arraia', } \\
\text { swelling, haemorrhoids } \\
\text { inflammation, wounds, } \\
\text { earache, erysipelas, } \\
\text { athlete's foot, tumour, } \\
\text { cancer }\end{array}$ & {$[7,8,42,73,143]$} \\
\hline
\end{tabular}


Table 1 Medicinal animals and its respective uses in popular medicine in Latin America (Continued)

Sylvilagus brasiliensis (Linnaeus, 1758) - Forest rabbit, tapeti

Sylvilagus floridanus (J. A. Allen, 1890)

Sylvilagus cunicularius (Waterhouse, 1848)

Oryctolagus cuniculus (Linnaeus, 1758) - Coelho

Lepus alleni Mearns, 1890

Sciuridae

Sciurus spadiceus Olfers, 1818

Sciurus deppei Peters, 1863

Sciurus aureogaster F. Cuvier, 1829

Ammospermophilus interpres (Merriam, 1890

\section{Mephitidae}

Conepatus semistriatus (Boddaert, 1785) - Striped hog-nosed skunk Skunk

Conepatus leuconotus leuconotus (Lichtenstein, 1832)

Mephitis macroura Lichtenstein, 1832

Thrombosis, conjunctivitis, $\quad[7,8,42,73,97,143,162]$

boils, burns,

ophthalmological

problems, embolism, scare,

fever, hallucination

Unspecified [152]

To bring good luck [103]

Unspecified

[4]

Stomach ache

[103]

$\begin{array}{ll}\text { Unspecified } & {[125]} \\ \text { Unspecified } & {[125]} \\ \text { Unspecified } & {[125]} \\ \text { Inflammation } & {[103]}\end{array}$

Rheumatism, acne, scabies, [7-9,42,73,162]

blood problems, bronchial

diseases, skin problems,

asthma, nervous

disturbances

Thrombosis, rheumatism, general diseases

Blood disorders, acne,

stomach ache, mal aire,

swelling, undescended

testicles, rabies, whooping

cough, bone pain Acné,

dolor muscular

Stomach ache, mal aire,

swelling, undescended

$[8,103,125,148]$

testicles, Rabies, whooping

cough, pain in bone,

asthma

Stomach ache, mal aire

swelling, undescended

testicles, rabies, whooping

cough, pain in bone

[91]

25]


Table 1 Medicinal animals and its respective uses in popular medicine in Latin America (Continued)

\begin{tabular}{|c|c|c|}
\hline Myrmecophaga tetradactyla (Linnaeus, 1758) - Collared anteater & $\begin{array}{l}\text { Edema, thrombosis, itching, } \\
\text { ant bite, rheumatism }\end{array}$ & {$[7-9,42,97]$} \\
\hline Tamandua mexicana (Saussure, 1860) - Northern Tamandua & Unspecified & {$[152]$} \\
\hline \multicolumn{3}{|l|}{ Procyonidae } \\
\hline Nasua nasua (Linnaeus, 1766) - South American coati & $\begin{array}{l}\text { Sexual impotence, wounds, } \\
\text { skin burns, snake bites, } \\
\text { backache, cold, cough, leg } \\
\text { pain, wounded foot, } \\
\text { earache, neck strain, to } \\
\text { help become pregnant, } \\
\text { whooping cough }\end{array}$ & {$[7-9,42,63,73,111,162,164,166]$} \\
\hline Nasua narica (Linnaeus, 1766) White-nosed Coati English & Male impotence & [152] \\
\hline Procyon cancrivorus (G. [Baron] Cuvier, 1798) - Crab-eating raccoon & $\begin{array}{l}\text { Rheumatism, epilepsy, } \\
\text { thrombosis, snake bite }\end{array}$ & {$[7-9,42,73,97,115]$} \\
\hline Procyon lotor (Linnaeus, 1758) & Unspecified & {$[152]$} \\
\hline Potos flavus (Schreber, 1774) - Kinkajou & $\begin{array}{l}\text { Earache, snake bite, ant } \\
\text { bite }\end{array}$ & {$[152]$} \\
\hline \multicolumn{3}{|l|}{ Physeteridae } \\
\hline Physeter catodon Linnaeus, 1758 - Sperm whale, cachelot & $\begin{array}{l}\text { Asthma, backache, } \\
\text { rheumatism, sore throat, } \\
\text { wounds }\end{array}$ & {$[7-9,42,119]$} \\
\hline \multicolumn{3}{|l|}{ Muridae } \\
\hline Neotoma mexicana Baird, 1855 & Unspecified & {$[38]$} \\
\hline Peromyscus mexicanus (Saussure, 1860) & Abdominal distension & {$[40]$} \\
\hline Mus musculus Linnaeus, 1758 & Pertussis & {$[175]$} \\
\hline \multicolumn{3}{|l|}{ Suidae } \\
\hline Sus scrofa (Linnaeus, 1758) - Wild boar & $\begin{array}{l}\text { Acne, boils, tumours, } \\
\text { asthma, athlete's foot, } \\
\text { warble, wounds, }\end{array}$ & {$[7-9,42,73,91]$} \\
\hline
\end{tabular}

\section{Tapiridae}

Tapirus terrestris (Linnaeus, 1758) - Brazilian tapir

Rheumatism, arthrosis,

$[8]$

osteoporosis, bursite,

muscular pain, asthma,

tonsillitis, cough, general

body pain

\begin{tabular}{|c|c|c|}
\hline Tapirus bairdii (Gill, 1865) & Unspecified & {$[152]$} \\
\hline \multicolumn{3}{|l|}{ Tayassuidae } \\
\hline Pecari tajacu Linnaeus 1758 - Collared peccary & $\begin{array}{l}\text { Thrombosis, bronchitis, } \\
\text { stroke }\end{array}$ & {$[8,85,115,162]$} \\
\hline Tayassu pecari (Link, 1795) - White-lipped peccary & $\begin{array}{l}\text { Thrombosis, stroke, cold, } \\
\text { wounds }\end{array}$ & {$[8,9,115,162]$} \\
\hline \multicolumn{3}{|l|}{ Trichechidae } \\
\hline Trichechus inunguis (Natterer, 1883) - Amazonian manatee & $\begin{array}{l}\text { Sprains, vaginal discharge, } \\
\text { injuries caused by bang, } \\
\text { burns, asthma, menstrual } \\
\text { cramps, rheumatism, sore } \\
\text { throat, wounds, muscle } \\
\text { strain, suck a splinter out } \\
\text { of skin or fresh, tumour, } \\
\text { backache, hernia, arthrosis, } \\
\text { luxation, insects bite }\end{array}$ & {$[7-9,31,42,155]$} \\
\hline Trichechus manatus (Linnaeus, 1758) - Manatee & $\begin{array}{l}\text { Arthrosis, luxation, } \\
\text { menstrual cramps, insects } \\
\text { bites, sprains, vaginal } \\
\text { discharge, injuries caused } \\
\text { by bang, burns, asthma, } \\
\text { rheumatism, sore throat, } \\
\text { wounds, muscle strain }\end{array}$ & {$[7-9,31,42,155]$} \\
\hline
\end{tabular}




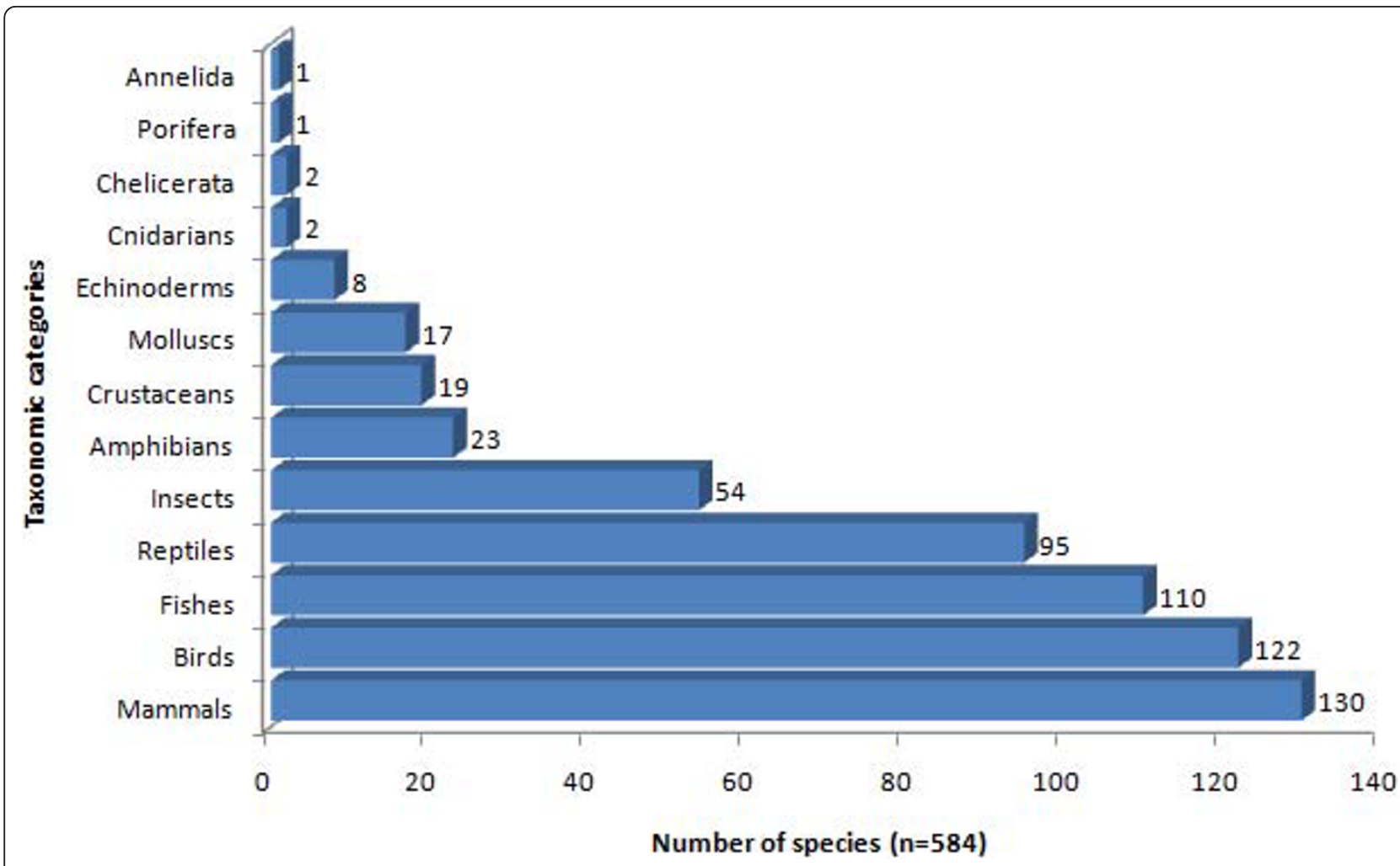

Figure 1 Number of animal species used as remedies per taxonomic category in Latin America.

Ingredients reportedly used in the preparation were fat, flesh, bone, bone marrow, cartilage, skin, tail, feather, liver, bile ("fel"), milk, rattle (from rattlesnakes), spine, shell, honey, wax, scale, rostral expansion, otolith, penis, carapace, blood, gizzard, beak, cocoon of insects, teeth, tongue, egg, egg shells, tibia, secretions, head, heart, urine, foot, legs, nest, guts, bezoar, ear, paw, spawn, nails, horn, sucking dish, eye, or more rarely, whole animals (Figures 3 and 4). Zootherapeutic products are consumed in several ways. Hard parts, such as teeth, nails, shells, rattles from snakes, fish scales, bone and cartilage generally are sun-dried, grated and crushed to powder, being then administered as tea or taken during meals, while fat, body secretion and oil are either ingested or used as an ointment. The influence of Westernization was also reflected in the presentation of some zootherapeutic products, which were either manufactured or pre-packaged. Examples are the fat extracted from the manatee (Trichecus sp.), sold as tablets, and the fat of Amazon River turtle (Podocnemis expansa (Schweiger, 1812)-Podocnemididae) sold as a manufactured soap in Brazil [42].

In many cases, the therapeutic use of animal remedies appears to be based on morphological or behavioral peculiarities of the animal in question. In México, for example, a tea made from the toasted and ground penis of the coatimundi (Nasua narica or N. nasua) is considered the most potent remedy for male impotence $[8,40]$. Descola [202] has recorded a similar remedy among the Achuar Jivaro of the Ecuadorian Amazon and provides an enlightening account of its probable origin: "The penis of the coati rejoices in a long fine bone that keeps it constantly rigid. This anatomical peculiarity has made a forceful impression upon the imagination of the Indians, and the men make the most of it, grating the bone into a decoction of green tobacco to make a love philtre. Quaffed at the right moment, it is reputed to prevent any flagging of the male member." In Brazil, reptiles that move slowly ("lerdos") are used to calm people ("lerdar"). For example, the products produced from $U$. superciliosus (dust and water) are used to "amansar" (to calm an aggressive person or ease the anger of someone betrayed by their wife or husband) [142]. There is an association between the biological characteristics of a lizard and the effects its use is expected to generate [134]. This observation is similar to that of Radbill [203] who pointed out that in homeopathic or imitative magic, it is assumed that certain qualities attributed to animals can be transferred to humans, and that this transfer can occur by inhalation, ingestion or application of the body parts of those animals. 

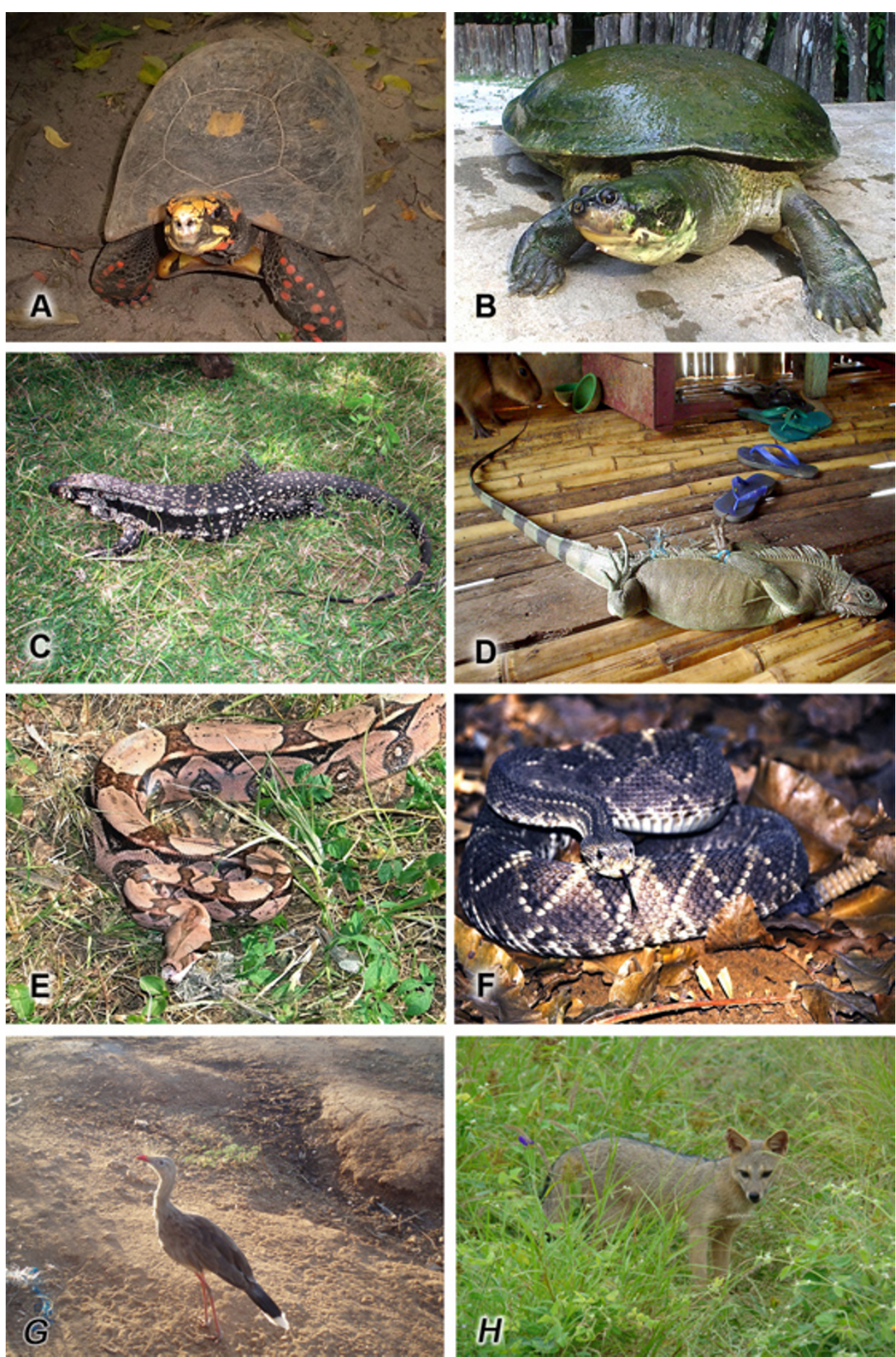

Figure 2 Examples of animals used as medicine in Latin America. A: Chelonoidis carbonaria, B: Podocnemis expansa, C - Tupinambis merianae, D - Iguana iguana, E - Boa constrictor, F - Caudisona durissa, G - Cariama cristata, H - Cerdocyon thous.

It is worth mentioning that such "natural modeling" does not necessarily preclude empirical efficacy. In Oxchuc, México, the two most common medicines given to speed delivery in cases of protracted labor are made from the toasted tail and shell of the nine-banded armadillo (Dasypus novemcinctus) and the tail of the Virginia opossum (Didelphis virginiana) [40]. Both animals are characterized by odd reproductive habits: 

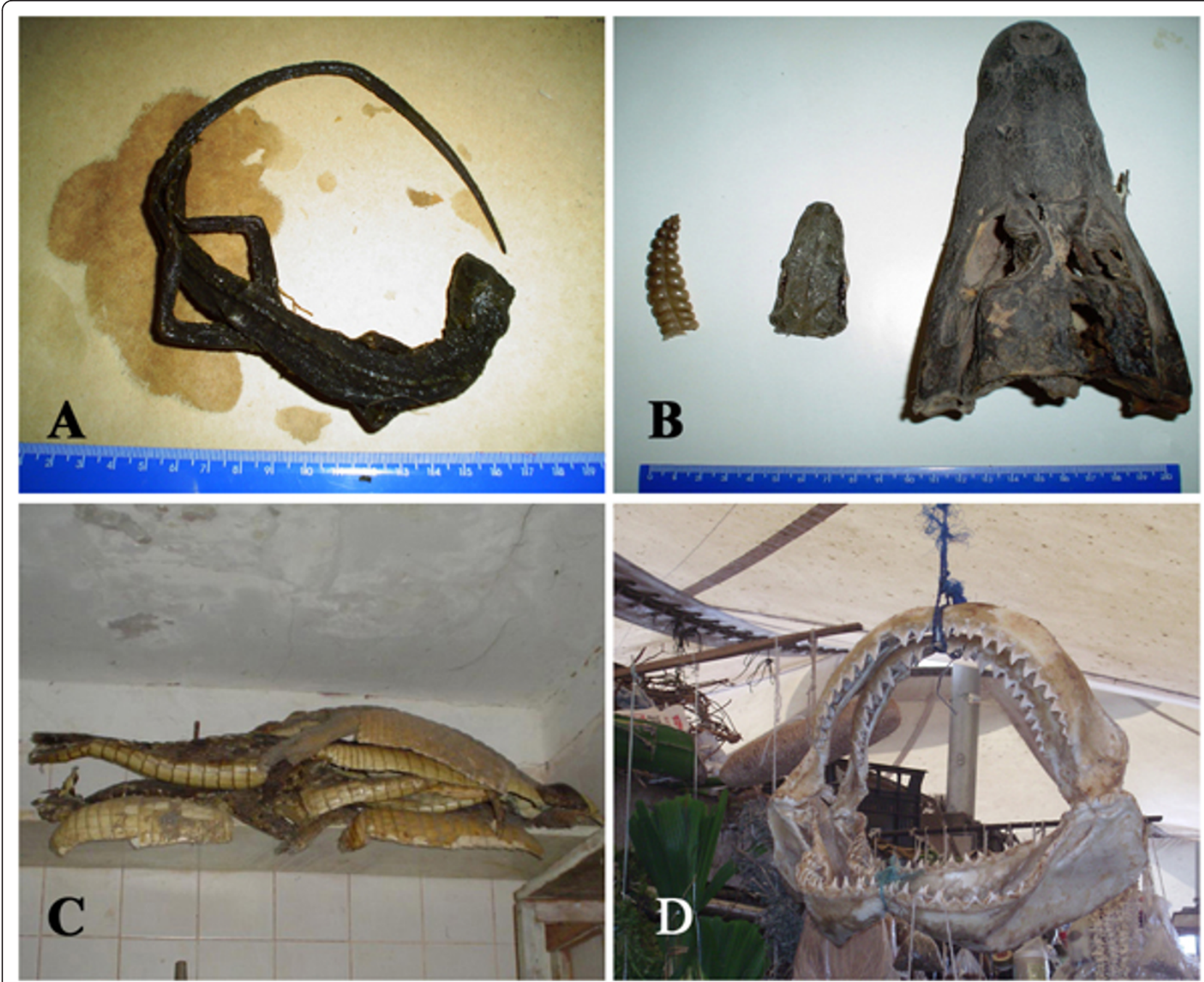

Figure 3 Examples of animal products used as remedies in Latin America (Brazil). A - Tamanquaré (Uranoscodon superciliosus), B - Rattle of rattlesnake (Caudisona durissa), boas' head (Boa constrictor) and cayman's head (Cayman sp), C - Cayman's skin (Cayman sp.) and D - Shark jaws and teeth.

Nine-banded armadillos regularly give birth to litters of identical quadruplets, and the opossum produces 10 to 20 offspring per year (no doubt owing to its 13-day gestation period, one of the shortest in the animal kingdom [204]. While these reproductive anomalies undoubtedly influenced their therapeutic use, the tail of the Virginia opossum has demonstrated uterotonic action in recent laboratory and clinical trials. This action probably derives from the presence of prostaglandins, which are known to be oxytocic in very small doses [205].

Some diseases affect both animals and humans and can be treated with similar remedies. This way, some animals are used in ethnoveterinary medicine and are also used for the treatment of human diseases. Barboza et al. [193] and Souto et al. [62] recorded the utilization of animals (zootherapeutics) as sources of medicines in folk veterinary medicine in semiarid northeast Brazil and verified that 46 animal species (43 vertebrates and 3 invertebrates) are used in the prevention or treatment of veterinary diseases in that region. Souto et al. [62] pointed that parallels between zootherapeutic practices in human and animal ethnomedicine not only include the types of animals used and the prevalence of use of those wildlife resources, but also in the modes of administration of these remedies and the ethnomedical techniques employed. This view of ethnomedicinal symbioses was highlighted by McCorkle and Martin [206], who noted that nearly all the ways in which ethnopharmaceuticals are administered (both externally and internally) are shared in animal and human treatments. Souto et al [62] recorded that local residents in the semi-arid region of Brazil administer zootherapeutic medicines topically (powders, ointment of fats, and others), or 


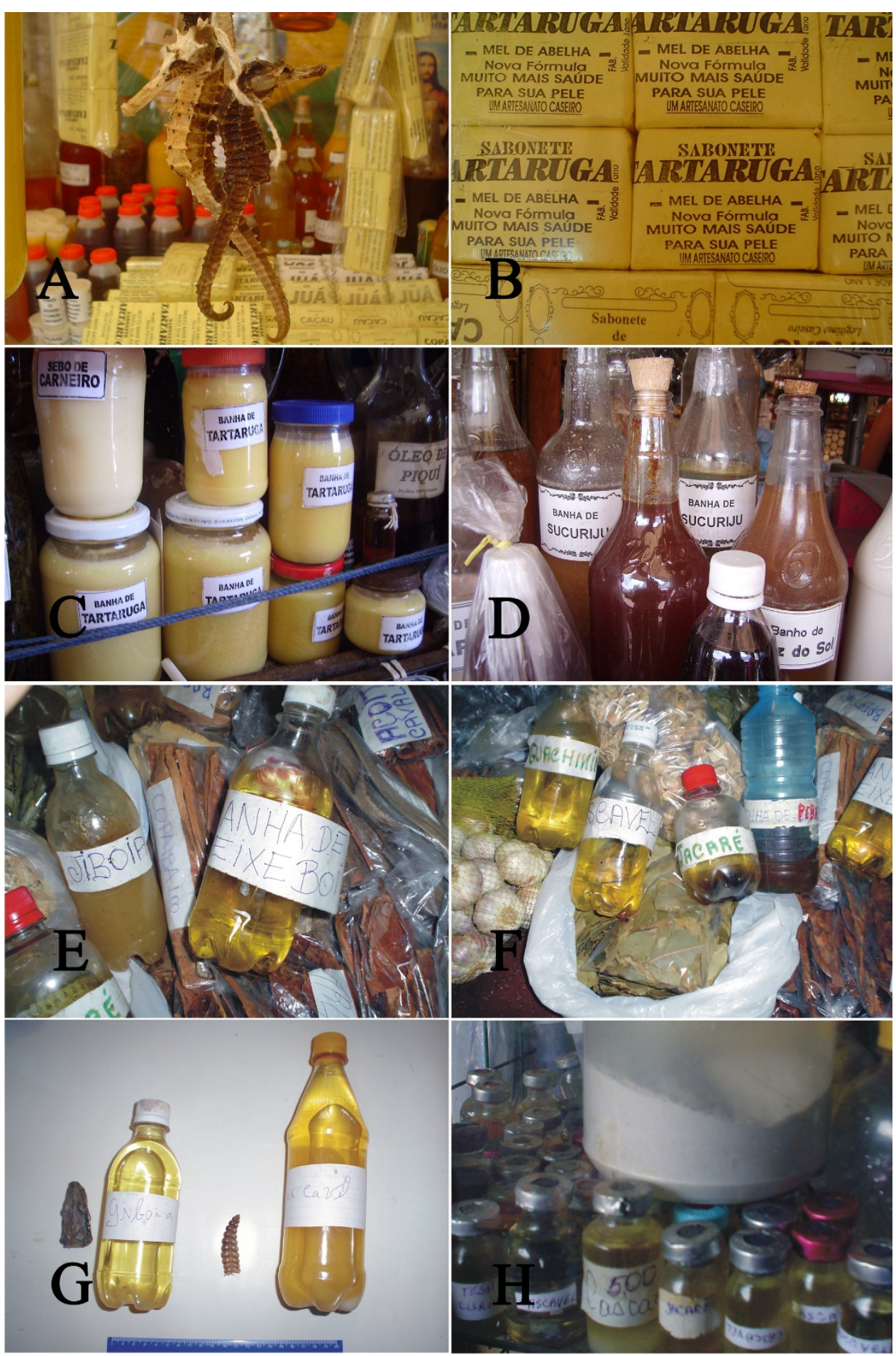

Figure 4 Medicinal animal products sold in Brazilian cities. A - Dried seahorses, B - Soap is produced from fat of turtle P. expansa and honey bee, C - Fat derived from sheep (Ovis aries) and Turtle (P. expansa), D - Anaconda fat (Eunectes murinus), E - Boa fats (Boa constrictor) and manatee (Trichecus sp.), F - Plastic bottles with raccoon fat (Procyon cancrivorus), rattlesnake (Caudisona durissa), caymans (Paleosuchus palpebrosus or Cayman crocodilus) and armadillo (Euphractus sexcintus), G - Head and fat of boa (B. constrictor) and rattle and fat of rattlesnake (C. durissa), and $\mathrm{H}$ : Oyster powder (Crassostrea rhizophorae), fats of different animals prompt to be commercialized right in big pots of plastic and in small flasks. 
orally to their livestock and pets in a manner very similar to human Complementary Alternative Medicine practices. The main techniques of preparing and administering animal-based remedies in local traditional medicine systems are commonly reported in Complementary and Alternative Medicinal practices in most parts of Brazil [7-9,31,132]. The close relationships between ethnoveterinary and human ethnomedicines can be easily explained from this perspective, as the main stock animals (e.g. cattle, sheep, goats, pigs, etc.) are mammals [132] that often have health problems very similar to humans and identical symptoms [62].

\section{Cultural beliefs}

Historically, traditional cultures recognized the importance of belief and expectancy within the healing encounter and created complex rituals and ceremonies designed to elicit or foster the expectancy and participation of healer and patient, as well as the community as a whole. Spiritual healing techniques have been a fundamental component of the healing rituals of virtually all societies since the advent of man $[207,208]$.

Folk-illnesses exist within the cultures that create them, "etiology, diagnosis, preventive measures and regimens of healing" [209] being provided by the culture. Cultural issues are recognized as important components of the provision of effective health care [210]. As noted by Straker [211], the supernatural world is as relevant in the diagnosis of illness as the natural world, with the main causes of illness being magical, mystical and animistic forces. Maher [212] showed that Aboriginal people have categorized illness as natural, environmental, direct supernatural, indirect supernatural and emergent or western causes. Pieroni and Quave [213] found, in a study carried at Ginestra/Zhure, Italy, that the aetiologies of various folk-illness are commonly linked to spiritual transmission, and that the treatments were often magical or psychotherapeutic in nature. Furthermore, for many illnesses deriving from relations with the supernatural, modern medicine is considered ineffective [214].

Besides their role in healing, natural products often have magical-religious significance, reflecting the different views of health and disease that exist within different cultures. In this context, animal parts are used to prepare clinical remedies as well as to make amulets or charms used in magical/religious diagnoses. Popular beliefs usually affect the way species are used in zootherapy $[7,8,29,134,146]$. One form of spiritual treatment involves the use of amulets containing reptile parts to protect the user from the "evil-eye" or from diseases. An example is caiman teeth (C. latirostris, $M$. niger, and $P$. palpebrosus) used as protection against snake bites [8].
The influence of magical beliefs integrated into traditional medicine is apparent [8]. In Brazil, for example, different animal species are used in magical-religious practices of Afro-Brazilian cults $[29,65,142,215,216]$ in the context of rituals that emphasize the holistic nature of traditional medicine and that are designed to address spiritual, physical, and social-psychological problems [42]. Because medical systems are organized as cultural systems, the use of animal substances should be understood according to a cultural perspective [64].

\section{Socio-economic aspects of zootherapy}

In addition to the belief systems, socioeconomic aspects also influence zootherapeutic practices [8]. Latin America has one of the greatest disparities in income distribution in the world. Overall, the health profile of the Latin American population can be classified as undergoing a slow epidemiological transition. At one extreme of the spectrum there is a high incidence of (and mortality from) chronic noninfectious diseases such as cardiovascular problems and cancer, which predominate in large metropolitan areas. On the other hand, infectious diseases still impose a heavy burden on the povertystricken parts of the population. The reasons for this dichotomy are two-fold: uneven socioeconomic development within countries and the extreme diversity of regional environments [217].

Studies suggest that Indigenous peoples of Latin America still have inadequate access to mainstream health services, and health prevention and promotion programmes, and that services that do exist are often culturally inappropriate $[218,219]$. The traditional medicine is widely available and affordable, yet in remote areas, and generally accessible to most people. In many developing countries, a large part of the population, especially in rural areas, depends mainly on traditional medicine for their primary health care, because it is cheaper and more accessible than orthodox medicine [8]. Traditional medicine is also more acceptable because it blends readily into the peoples' socio-cultural belief system [220-222]. Hence, resorting to the use of medicinal animals and plants, which were easily accessible and relatively cheap is an important component to healthcare in these gettings [8].

\section{Health Concerns}

Traditional drugs and traditional medicine in general represent a still poorly explored field of research in terms of therapeutic potential or clinical evaluation. There is a current preoccupation about this, since it is well-established that all sorts of vegetable, animal and mineral remedies used in a traditional setting are capable of producing serious adverse reactions. It is essential, however, that traditional drug therapies be 
submitted to an appropriate benefit/risk analysis. De Smet [223] draws attention to the fact that some sideeffects of traditional medicines have proven to be more severe than the disease that they are treating. Such considerations must be taken into account, further highlighting the need for clinical studies of traditional remedies.

Numerous infectious diseases can be transmitted from animals to humans [224]. Zoonoses constitute other animal health problems that affect public health. These represent an important threat for the welfare of human populations. In the urban and rural areas of the countries under development, zoonoses continue to present high frequencies [225]. In this context, the possibility of transmitting infections or ailments from animal preparations to the patient should be seriously considered [224]. Several organs and tissues including bones and bile can be a source of Salmonella infection causing chronic diarrhoea and endotoxic shock. The possibility of transmission of other serious and widespread zoonoses such as tuberculosis or rabies should be considered whenever animal tissues from unknown sources are handled and used as remedies [226].

Several species of animals have become a favorite target of hunters because its bones, organs and tissues are used in traditional medicine $[136,143]$. Indeed, numerous species of animals are hunted (poached) rather for their meat than for their supposed medical use $[136,140]$. Nevertheless, there is often an overlap between the two purposes, and transmission of disease can occur in both instances. One excellent example of this regards the primates [140]. Several species of monkeys have been identified as harboring infectious diseases transmissible to man with potentially grave consequences [224,227]. The related risks can be illustrated by a recent case from a Safari Park in Great Britain, where a colony of otherwise clinically healthy Macaque monkeys had to be humanely destroyed once it had been discovered that the monkeys harbored the simian herpes B virus [228]. While the agent is not harmful to monkeys, it causes mortality in $80 \%$ of affected humans. Certain researchers seriously consider the hypothesis that the worldwide epidemic of the HIV virus (causing the AIDS infection in humans) might have been initiated by transmission of a virus from monkeys several decades earlier [229].

The effectiveness of most of the medicines from wild animals/wild animal parts has not been scientifically studied and proven and their potency in many cases may be questionable. As pointed out by Pieroni et al. [230] the chemical constituents and pharmacological actions of some animal products are already known to some extent and ethnopharmacological studies focused on animal remedies could be very important in order to clarify the eventual therapeutic usefulness of this class of biological remedies. However, research with therapeutic purposes into the products of the animal kingdom has been neglected until recently [25,231]. In comparison to research done on plant drugs, modern pharmacology has done far fewer studies on animal products and minerals [232].

Historically, traditional use of plants as medicines has been extensively recognized, studied, and reviewed. However, such attention has not been paid to the potential of animals as a source of medicinally relevant substances. A excellent example of this potential is provided by insects. Trowell [233] points out that there are at least 16 times as many insect species as there are plant species, yet plant chemistry has been studied 7000 times as much as insect chemistry when comparing the amount of research per species. Nonetheless, the vast biodiversity which exists in the arthropod world, compared to all other organisms on earth, certainly suggests that arthropods should be given a more serious look [234]. Nevertheless, in last years, there has been increasing attention paid to animals, both vertebrates and invertebrates, as sources for new medicines [235].

\section{Environmental Approach}

Various authors have discussed the conservation implications of the use of medicinal products derived from wild animal species $[16,33,34,143,236]$. In Latin America, as evidenced in the present review, the medicinal fauna is largely based on wild animals, including many endangered species.

The use of medicinal animals is common in both rural and urban areas. Biological remedies are openly commercialized in towns and cities, principally in public markets. It is common to find specific places in these markets where plants and animals are sold for medicinal purposes [31,32,38,42,66,67,131,133,135,138,144,237]. The commercialization of animals for medicinal purposes is a widespread phenomenon, with significant implications for their conservation and sustainable use [1]. Previous authors [238-242] have suggested that market expansion induces people to make greater use of wild animals for traditional medicine and that the practice has spread in developed nations of Asia and the Pacific (e.g., Taiwan, Australia). But other research suggests that the increasing use of animals for traditional medicine can also take place without economic prosperity. For example, Kritsky [242] reported that the use of insects for traditional medicine in China increased during the Cultural Revolution. Based on an ethno-zoological survey of the use of medicinal birds, Joseph [243] concludes that the use of birds to treat human ailments increased in Madhya Pradesh, Central India, because people could not afford modern treatments. 
The worldwide market for animal parts and their medicinal derivatives is contributing to the loss of some species. The increased use of medicinal animals has led to overexploitation of species like rhinos, tigers, musk deer, bears, monkeys and pangolins. In spite of international regulations and several national laws against poaching and heavy penalties for culprits, the extremely high prices offered for the parts of some species serve as strong incentives for illegal trade in animal parts to flourish $[1,2]$.

It must be emphasized, however, that many factors affect animal populations in the world, and the use of these animals for medicinal purposes is only part of the problem. As such, the medicinal use of animals must be considered together with other anthropogenic pressures, such as habitat loss. The depletion of medicinal resources not only poses a challenge for conservation but represents a serious threat to the health of many human communities, and that efforts to stabilize the status of these species are important not only to conservationists but to millions of people whose health depends of the use of traditional remedies [136,140,224]. Moura and Marques [74] pointed that one characteristic in common among all zootherapeutic products, whether whole animals or their parts, is their lack of use for other purposes. In this sense, it is remarkable that in most cases, the medicinal products of animals are byproducts from animals hunted for other purposes; thus, these multiple uses (including medicinal) of fauna and their impact on animal populations must be properly assessed and taken into consideration when implementing recovery plans for these species, especially those that are highly exploited [7-9,31,140]. Medicinal species whose conservation status is a cause of concern should receive urgent attention, and aspects such as habitat loss/alteration should be discussed in connection with present and future use of these species in folk medicine [8]

Zootherapy is intertwined with sociocultural and religious beliefs that must be understood by those engaged in modern conservation and protection of biodiversity. Celso [244] pointed out that natural medicine is one important use of biodiversity. Some traditional medicinal systems, like the Chinese Traditional Medicine, is recognized by the World Health Organization (WHO) and accepted by one-fourth of the world human population, and the reliance on traditional medicinal uses of animals by communities around the world should be addressed when designing strategies to conserve biodiversity. Conservation permits the continuing use of the resources in ways that are non-destructive and sustainable, while from the pharmaceutical point of view, it provides time to eventually demonstrate fully the potentially medicinal value of the resources [245].
Connections between traditional medicine, biodiversity and human health have recently been addressed by different authors [1,22,246-248]; have drawn attention to the fact that biodiversity loss can have indirect and direct effects on human well-being as well. The reliance on traditional uses of animals as food and as medicine by communities around the world highlights the need for further interdisciplinary research in ethnozoology which can be used in strategies to conserve biodiversity $[53,249]$. Furthermore, loss of wildlife resources, aside from threatening people's health and well-being, affects their cultural integrity. In Latin America, despite the many individual efforts of the governments to preserve the biodiversity for future generations, traditional knowledge, especially that derived from indigenous knowledge (such as Traditional Medicine), is also disappearing [45]. In this sense, understanding the contexts of traditional therapeutic uses of animals, is central for elucidating their potential impact in public health and biodiversity conservation.

\section{Conclusions}

Latin America has a wealth of biological resources and is home to a large number of different ethnic and cultural groups, many of which have developed their own, distinct health care systems. As a result, the region is rich in traditional medicinal knowledge and zootherapy represents an alternative to official medicinal practices in rural areas and has also become part of urban popular medicine. Our results reveal that at least 584 animals are used for medicinal purposes in Latin America, underlining the importance of zootherapy as alternative therapeutic in region.

Animals provide the raw materials for remedies used to treat physical and/or spiritual diseases. Besides being influenced by cultural aspects, the relations between humans and biodiversity in the form of zootherapeutic practices are conditioned by the social and economic relations between humans themselves. In a region like Latin America, where the majority of the population has no access allopathic medicine, local medicinal animals and plant knowledge systems is of significance. The population uses traditional medicine due to the relatively low cost and difficult access to modern health facilities. Nevertheless, the interest in and intrinsic value of zootherapy not be only be attributed to the lack of access to modern medicinal services. Even in cities where modern health services are more accessible and specialized; many people continue to go to traditional healers showing the cultural acceptability of such practices.

Threatened species represented important medicinal resources in Latin America. This shows the need to 
integrate traditional knowledge into strategies to conserve and manage faunistic resources. Sustainability of harvesting of medicinal animals is challenged by many factors, from both social and ecological perspectives. It is important to respect differing views of the value of wildlife, while, at the same time, conserving biodiversity.

Using animal products as components of bioprospecting has implications for medicine, the environment, economy, public health and culture. Although widely diffused, zootherapeutic practices remain virtually unstudied, and so far there has been neither a demonstration of the clinical efficacy of the popularly used remedies nor an evaluation of the sanitary implications of the prescription of animal products for the treatment of diseases in the Latin America. New studies of medicinal fauna, which seek a better understanding of this form of therapy - including ecological, cultural and pharmacological aspects, are necessary.

\section{Author details \\ ${ }^{1}$ Departamento de Biologia, Universidade Estadual da Paraíba, Avenida das Baraúnas, Campina Grande, Paraíba 58109-753, Brasil. ²Prefeitura Municipal de João Pessoa, Escola Municipal Arnaldo de Barros Moreira, Rua Capitão Francisco Pereira, 365, Bairro dos Novais, João Pessoa, Paraíba, 58088-530, Brasil.}

\section{Authors' contributions}

RRNA and HNA worked in the bibliographical classification, conception and the article final composition. The authors read and approved the final manuscript.

\section{Competing interests}

The authors declare that they have no competing interests.

Received: 12 January 2011 Accepted: 7 March 2011

Published: 7 March 2011

\section{References}

1. Alves RRN, Rosa IL: Biodiversity, traditional medicine and public health: where do they meet? Journal of Ethnobiology and Ethnomedicine 2007, 3:9.

2. Alves RRN, Rosa IL: Why study the use of animal products in traditional medicines? Journal of Ethnobiology and Ethnomedicine 2005, 1:1-5.

3. WHO - World Health Organization: Traditional Medicine Strategy 20022005.[http://whqlibdoc.who.int/hq/2002/who_edm_trm_2002.1.pdf].

4. Costa-Neto EM, Alves RRN: Zooterapia: Os Animais na Medicina Popular Brasileira. 1 edition. Recife, PE, Brazil: NUPEEA; 2010.

5. Agra MF, Baracho GS, Nurit K, Basílio IJLD, Coelho VPM: Medicinal and poisonous diversity of the fora of "Cariri Paraibano", Brazil. Journal of Ethnopharmacology 2007, 111:383-395.

6. Uniyal SK, Singh KN, Jamwal P, Lal B: Traditional use of medicinal plants among the tribal communities of Chhota Bhangal, Western Himalaya. J Ethnobiol Ethnomed 2006, 2:14.

7. Alves RRN, Rosa IL: From cnidarians to mammals: The use of animals as remedies in fishing communities in NE Brazil. Journal of Ethnopharmacology 2006, 107:259-276.

8. Alves RRN, Rosa IL, Santana GG: The Role of Animal-derived Remedies as Complementary Medicine in Brazil. BioScience 2007, 57:949-955.

9. Alves RRN, Rosa IL: Zootherapeutic practices among fishing communities in North and Northeast Brazil: A comparison. Journal of Ethnopharmacology 2007, 111:82-103.

10. Alves R, Filho GAP, De Lima YCC: Snakes used in ethnomedicine in Northeast Brazil. Environment, Development and Sustainability 2007, 9:455-464.

11. Alves RRN: Fauna used in popular medicine in Northeast Brazil. Journal of Ethnobiology and Ethnomedicine 2009, 5:1-30.
12. Kunwar R, Bussmann RW: Ethnobotany in the Nepal Himalaya. J Ethnobiol Ethnomed 2008, 4:24

13. Pradhan BK, Badola HK: Ethnomedicinal plant use by Lepcha tribe of Dzongu valley, bordering Khangchendzonga Biosphere Reserve, in North Sikkim, India. J Ethnobiol Ethnomed 2008, 4:22.

14. Napoli M: The plants, rituals and spells that 'cured' helminthiasis in Sicily. J Ethnobiol Ethnomed 2008, 4:21.

15. Yineger $H$, Yewhalaw D, Teketay D: Ethnomedicinal plant knowledge and practice of the Oromo ethnic group in southwestern Ethiopia. J Ethnobiol Ethnomed 2008, 4:11

16. Mahawar MM, Jaroli DP: Traditional zootherapeutic studies in India: a review. Journal of Ethnobiology and Ethnomedicine 2008, 4:17.

17. Voultsiadou E: Therapeutic properties and uses of marine invertebrates in the ancient Greek world and early Byzantium. Journal of Ethnopharmacology 2010, 130:237-247.

18. Yesilada E: Past and future contributions to traditional medicine in the health care system of the Middle-East. J Ethnopharmacol 2005, 100:135-137.

19. Lev $E$, Amar Z: Ethnopharmacological survey of traditional drugs sold in the Kingdom of Jordan. Journal of Ethnopharmacology 2002, 82:131-145.

20. Lev E: Healing with animals in the Levant from the 10th to the 18th cent. Journal of Ethnobiology and Ethnomedicine 2006, 2:9.

21. Lev E: Traditional healing with animals (zootherapy): medieval to present-day Levantine practice. Journal of Ethnopharmacology 2003, 85:107-118.

22. Chivian E: Biodiversity: Its Importance to Human Health Cambridge, USA: Center for Health and the Global Environment. Harvard Medical School; 2002.

23. Unnikrishnan E: Materia Medica of the Local Health Traditions of Payyannur Centre for Development Studies; 2004.

24. Alakbarli F: Medical manuscripts of Azerbaijan Baku Heydar Aliyev Foundation; 2006.

25. Scarpa A: Pre-scientific medicines: their extent and value. Soc Sci Med 1981, 15A:317-326.

26. Marques JGW: A fauna medicinal dos índios Kuna de San Blas (Panamá) e a hipótese da universalidade zooterapica. Anais da $46 a$ Reunião Anual da SBPC 1994, 324

27. Quave CL, Lohani U, Verde A, Fajardo J, Rivera D, Obón C, Valdes A, Pieroni A: A comparative assessment of zootherapeutic remedies from selected areas in Albania, Italy, Spain and Nepal. Journal of Ethnobiology 2010, 30:92-125.

28. Ibrahim JA, Muazzam I, Jegede IA, Kunle OF: Medicinal plants and animals sold by the "Yan-Shimfidas" of Sabo Wuse in Niger State, Nigeria. African Journal of Pharmacy and Pharmacology 2010, 4:386-394.

29. Alves RRN, Pereira Filho GA: Commercialization and use of snakes in North and Northeastern Brazil: implications for conservation and management. Biodivers Conserv 2007, 16:969-985.

30. Almeida CFCBR, Albuquerque UP: Uso e conservação de plantas e animais medicinais no Estado de Pernambuco (Nordeste do Brasil): Um estudo de caso. Interciencia 2002, 27:276-285.

31. Alves RRN, Rosa IL: Zootherapy goes to town: The use of animal-based remedies in urban areas of NE and N Brazil. Journal of Ethnopharmacology 2007, 113:541-555.

32. Apaza L, Godoy R, Wilkie D, Byron E, Huanca T, Leonard WR, Peréz E, ReyesGarcía V, Vadez V: Markets and the use of wild animals for traditional medicine: a case study among the Tsimané ameridians of the Bolivian rain forest. Journal of Ethnobiology 2003, 23:47-64.

33. Ashwell D, Walston N: An overview of the use and trade of plants and animals in traditional medicine systems in Cambodia. 1 edition. Ha Noi, Vietnam: TRAFFIC Southeast Asia, Greater Mekong Programme; 2008.

34. Kang S, Phipps M: A question of attitude: South Korea's Traditional Medicine Practitioners and Wildlife Conservation. 1 edition. Hong Kong: TRAFFIC East Asia; 2003.

35. Pieroni A, Giusti ME, Grazzini A: Animal remedies in the folk medicinal practices of the Lucca and Pistoia Provinces, Central Italy. In Des sources du savoir aux médicaments du futur/from the sources of knowledge to the medicines of the future. 1 edition. Edited by: Fleurentin J, Pelt JM, Mazars G. Paris: IRD Editions; 2002:371-375.

36. Silva MLV, Alves ÂGC, Almeida AV: A zooterapia no Recife (Pernambuco): uma articulação entre as práticas e a história. Biotemas 2004, 17:95-116 
37. Sodeinde OA, Soewu DA: Pilot study of the traditional medicine trade in Nigeria. Traffic Bulletin 1999, 18:35-40.

38. Vázquez PE, Méndez RM, Guiascón ÓGR, Piñera EJN: Uso medicinal de la fauna silvestre en los Altos de Chiapas, México. Interciencia 2006, 31:491-499.

39. Van NDN, Tap N: An overview of the use of plants and animals in traditional medicine systems in Viet Nam. 1 edition. Ha Noi, Viet Nam: TRAFFIC Southeast Asia, Greater Mekong Programme; 2008.

40. Groark KP: To Warm the Blood, To Warm the Flesh: The Role of the Steambath in Highland Maya (Tzeltal-Tzotzil) Ethnomedicine. Journal of Latin American Lore 1997, 20:3-96.

41. Alves RRN: O comércio de recursos zooterápicos. In Zooterapia: Os Animais na Medicina Popular Brasileira. Volume 2. 1 edition. Edited by: CostaNeto EM, Alves RRN. Recife, PE, Brazil: NUPEEA; 2010:159-176.

42. Alves RRN, Rosa IL: Trade of animals used in Brazilian traditional medicine: trends and implications for conservation. Human Ecology 2010, 38:691-704.

43. Alves RRN, Barboza RRD, Souto WMS: Plants Used in Animal Health Care in South and Latin America: An Overview. In Ethnoveterinary Botanical Medicine: Herbal Medicines for Animal Health. 1 edition. Edited by: Katerere RD, Luseba D. New York, USA: CRC Press; 2010:231-256.

44. Hauser G, Little M, Roberts DF: Man, Culture and Biodiversity: Understanding interdependences Paris: IUBS; 1994.

45. Calixto JB: Twenty-five years of research on medicinal plants in Latin America: A personal view. Journal of Ethnopharmacology 2005, 100:131-134.

46. Kent RB: Latin America: Regions and People New York and London: The Guilford Press; 2006.

47. Leo Neto NA, Alves RRN: A Natureza sagrada do Candomblé: análise da construção mística acerca da Natureza em terreiros de candomblé no Nordeste de Brasil. Interciencia 2010, 35:568-574.

48. Dias TLP, Leo Neto NA, Alves RRN: Molluscs in the marine curio and souvenir trade in NE Brazil: species composition and implications for their conservation and management. Biodiversity and Conservation 2011.

49. Fernandes-Ferreira $\mathrm{H}$, Mendonça SV, Albano C, Ferreira FS, Alves RRN: Comércio e criação de aves silvestres (Psittaciformes, Piciformes e Passeriformes) no Estado do Ceará. In A Etnozoologia no Brasil: Importância, Status atual e Perspectivas. Volume 7.1 edition. Edited by: Alves RRN, Souto WMS, Mourão JS. Recife, PE, Brazil: NUPEEA; 2010:379-402.

50. Alves RRN, Nishida AK: A ecdise do caranguejo-uçá, Ucides cordatus L. (Decapoda, Brachyura) na visão dos caranguejeiros. Interciencia 2002, 27:110-117.

51. Alves RRN, Nishida A, Hernandez M: Environmental perception of gatherers of the crab 'caranguejo-uca' (Ucides cordatus, Decapoda, Brachyura) affecting their collection attitudes. Journal of Ethnobiology and Ethnomedicine 2005, 1:10.

52. Oliveira ES, Torres DF, Alves RRN, Vasconcellos A: Etnozoologia em áreas protegidas: uso da fauna por populações locais na APA Bonfim/ Guaraíras, Rio Grande do Norte, Brasil. In A Etnozoologia no Brasil: Importância, Status atual e Perspectivas. Volume 7. 1 edition. Edited by: Alves RRN, Souto WMS, Mourão JS. Recife, PE, Brazil: NUPEEA; 2010:403-422.

53. Alves RRN, Souto WMS, Mourão JS: A Etnozoologia no Brasil: Importância, Status atual e Perspectivas. 1 edition. Recife, PE, Brazil: NUPEEA; 2010.

54. Nishida AK, Nordi N, Alves RRN: Mollusc Gathering in Northeast Brazil: An Ethnoecological Approach. Human Ecology 2006, 34:133-145.

55. Souto WMS, Barboza RRD, Mourão JS, Alves RRN: Zootherapy in Brazil: An Urgent Necessity of Interdisciplinary Studies. West Indian Medical Journal 2009, 58:494-495.

56. Alves RRN, Nogueira E, Araujo H, Brooks S: Bird-keeping in the Caatinga, NE Brazil. Human Ecology 2010, 38:147-156.

57. Rocha MSP, Mourão JS, Souto WMS, Barboza RRD, Alves RRN: Uso dos recursos pesqueiros no Estuário do Rio Mamanguape, Estado da Paraíba, Brasil. Interciencia 2008, 33:903-909.

58. Bennett EL, Robinson JG: Hunting of Wildlife in Tropical Forests. The World Bank Environment Department Papers 2000, 1-42.

59. Robinson JG, Redford KH: Neotropical wildlife use and conservation University of Chicago Press, Chicago; 1991.

60. Population Reference Bureau: World Population Highlights 2007: Overview of World Population. 2008 [http://www.prb.org/Articles/2007/ 623WorldPop.aspx], acessed 01 february 2011.
61. Field listing-ethnic groups. [https://www.cia.gov/library/publications/theworld-factbook/fields/2075.html].

62. Souto W, Mourao JS, Barboza RRD, Alves RRN: Parallels between zootherapeutic practices in Ethnoveterinary and Human Complementary Medicine in NE Brazil. Journal of ethnopharmacology 2011.

63. Nomura H: Folclore dos anfíbios Recife: Fundação Joaquim Nabuco; 1989

64. Costa-Neto EM: Implications and applications of folk zootherapy in the state of Bahia, Northeastern Brazil. Sustainable Development 2004, 12:161-174.

65. Alves RRN, Pereira-Filho GA, Vieira KS, Santana GG, Vieira WLS, Almeida WO: Répteis e as populações humanas no Brasil:uma abordagem etnoherpetológica. In A Etnozoologia no Brasil: importância, status atual e perspectivas futuras Edited by: Alves RRN, Souto WMS, Mourão JS. Recife: NUPEEA; 2010:121-146.

66. Costa-Neto EM: Traditional use and sale of animals as medicines in Feira de Santana City, Bahia, Brazil. Indigenous Knowledge and Development Monitor 1999, 7:6-9.

67. Costa-Neto EM: Animal Species Traded as Ethnomedicinal Resources in the Federal District, Central West Region of Brazil. The Open Complementary Medicine Journal 2010, 2:24-30.

68. De la Galvez Murillo E, Pacheco LF: Short Communication Abundancia y estructura poblacional de la lagartija jararank'o (Liolaemus signifer; Liolaemidae-Lacertilia-Reptilia) en zonas con y sin extracción comercial en el Altiplano de Bolivia. Tropical Conservation Science 2009, 2:106-115.

69. Alves RRN, Barbosa JAA, Santos SLDX, Souto WMS, Barboza RRD: Animalbased Remedies as Complementary Medicines in the Semi-arid Region of Northeastern Brazil. Evidence-Based Complementary and Alternative Medicine 2011, 2011:1-15

70. Torres DF, Oliveira ES, Alves RRN, Vasconcellos A: Etnobotânica e Etnozoologia em Unidades de Conservação: Uso da biodiversidade na Apa de Genipabu, Rio Grande do Norte, Brasil. Interciencia 2009, 34:623-629.

71. Begossi A: Food taboos at Búzios Island (SE Brazil): their significance and relation to folk medicine. Journal of Ethnobiology 1992, 12:117-139.

72. Figueiredo N: Os 'bichos' que curam: os animais e a medicina 'folk' em Belém do Pará. Boletim do Museu Paraense Emílio Göeldi 1994, 10:75-91.

73. Branch L, Silva MF: Folk medicine in Alter do Chão, Pará, Brasil. Acta Amazônica 1983, 13:737-797.

74. Moura FBP, Marques JGW: Zooterapia popular na Chapada Diamantina: uma Medicina incidental? Ciência \& Saúde Coletiva 2008, 13:2179-2188.

75. Costa-Neto EM: A zooterapia popular no Estado da Bahia: registro de novas espécies animais utilizadas como recursos medicinais. Revista Ciência \& Saúde Coletiva 2009, 1:1-2.

76. Alves RRN, Pereira Filho GA: Commercialization and use of snakes in North and Northeastern Brazil: implications for conservation and management. In Vertebrate Conservation and Biodiversity. 1 edition. Edited by: Hawksworth DL, Bull AT. Amsterdan: Springer Netherlands; 2007:143-159.

77. Barros FB, Pereira HM, Vicente L: Use and Knowledge of the Razor-billed Curassow Pauxi tuberosa (Spix, 1825) (Galliformes, Cracidae) by a Riverine Community of the Oriental Amazonia, Brazil. Journal of Ethnobiology and Ethnomedicine 2011, 7:1.

78. Rosa IL, Alves RRN, Bonifacio K, Mourão JS, Osorio F, Oliveira T, Nottingham M: Fishers' knowledge and seahorse conservation in Brazil. Journal of Ethnobiology and Ethnomedicine 2005, 1:1-12.

79. Baum JK, Vincent ACJ: Magnitude and inferred impacts of the seahorse trade in Latin America. Environmental Conservation 2005, 32:305-319.

80. Bayúgar AC: Conocimiento y comparación del uso de la fauna silvestre en dos comunidades Ejidales del municipio de Hueytamalco, Puebla, Mexico. Dissertação de Mestrado Instituto de Ecologia; 2007.

81. Herrera JG: Percepción geoecológica sobre la fauna silvestre: hacia una mejor estrategia de manejo local. Universidad Nacional Autónoma de México; 2008.

82. Barboza RSL: Interface conhecimento tradicional-conhecimento científico: um olhar interdisciplinar da etnobiologia na pesca artesanal em Ajuruteua, Bragança-Pará. Dissertação de Mestrado Universidade Federal do Pará; 2008.

83. Sousa RS: Etnobotânica e etnozoologia de comunidades pesqueiras da Área de Proteção Ambiental (APA) do Delta do Parnaíba, Nordeste do Brasil. Dissertação de Mestrado Universidade Federal do Piauá; 2010. 
84. Silva NLG, Ferreira FS, Coutinho HDM, Alves RRN: Zooterápicos utilizados em comunidades rurais do município de Sumé, semiárido da Paraíba, Nordeste do Brasil. In Zooterapia: Os Animais na Medicina Popular Brasileira. Volume 2. 1 edition. Edited by: Costa-Neto EM, Alves RRN. Recife, PE, Brazil: NUPEEA; 2010:243-267.

85. Silva AL: Animais medicinais: conhecimento e uso entre as populações ribeirinhas do rio Negro, Amazonas, Brasil. Boletim do Museu Paraense Emílio Göeldi 2008, 3:343-357.

86. Shepard GH: Primates in Matsigenka subsistence and world view. In Primates face to face: conservation implications of human-nonhuman primate interconnections. Volume 101-136. Edited by: Fuentes A, Wolfe L. Cambridge: Cambridge University Press; 2002:101-136.

87. SEMMA: Plano de Manejo da Reserva de Desenvolvimento Sustentável do Tupé. Book Plano de Manejo da Reserva de Desenvolvimento Sustentável do Tupé Prefeitura Municipal de Manaus; 2008.

88. Seixas C, Begossi A: Ethnozoology of caiçaras from Aventureiro, llha Grande. Journal of Ethnobiology 2001, 21:107-135.

89. Rodrigues ER: Conhecimento etnoentomológico sobre abelha indígena sem ferrão (Melipona) e meliponicultura na comunidade de São Pedro dos Bois do estado do Amapá. Universidade Federal do Amapá; 2009.

90. Rodrigues E: Plants and Animals Utilized as Medicines in the Jaú National Park (JNP), Brazilian Amazon. Phytotherapy Research 2006, 20:378-391.

91. Ribeiro GC, Pereira JPR, Docio L, Alarcon DT, Schiavetti A: Zooterápicos utilizados no sul da Bahia. In Zooterapia: Os Animais na Medicina Popular Brasileira. Volume 2. 1 edition. Edited by: Costa-Neto EM, Alves RRN. Recife, PE, Brazil: NUPEEA; 2010:221-242.

92. Ribeiro GC: Atitudes de conservação e conhecimento dos moradores da região do Parque Estadual da Serra do Conduru (Bahia) sobre mamíferos arborícolas. Dissertação de Mestrado Universidade Estadual de Santa Cruz; 2008.

93. Pereira JPR, Schiavetti A: Conhecimentos e usos da fauna cinegética pelos caçadores indígenas "Tupinambá de Olivença" (Bahia). Biota Neotropica 2010, 10:175-183.

94. Ornelas MLN: Presencia e importancia de los animales en la medicina tradicional de los grupos otopames. Estudios de Cultura Otopame 2010, 4:197-214

95. Moura FBP, Marques JGW: Zooterapia popular na Chapada Diamantina: uma Medicina incidental? Ciência \& Saúde Coletiva 2008, 13:2179-2188.

96. Morales-Mavil JE, Villa-Cañedo JT: Notas sobre el uso de la fauna silvestre en Catemaco, Veracruz, México. Acta Zoologica Mexicana 1998, 73:127-143.

97. Monroy-Vilchis O, Cabrera L, Suárez P, Zarco-González MM, RodríguezSoto C, Urios V: Uso tradicional de vertebrados silvestres en la Sierra Nanchititla, México. Interciencia 2008, 33:308-313.

98. Mittermeier RA: Effects of hunting on rain forest primates. In Primate Conservation in the Tropical Rain Forest Edited by: Marsch CW, Mittermeier R. New York: Alan R. Liss Inc; 1987:109-146.

99. Martínez C: Las aves como recurso curativo en el México antiguo y sus posibles evidencias en la arqueozoología. Arqueobios 2008, 2:11-18.

100. Marques JGW: Pescando pescadores: etnoecologia abrangente no baixo São Francisco alagoano São Paulo, BR: NUPAUB-USP; 1995.

101. Marques JGW: Insects as folk medicines in the State of Alagoas, Brazil. Insect Food Newsletter 1995, 1:75.

102. Mallmann MLW: A farmacopéia do mar: invertebrados marinhos de interesse médico e a etnomedicina alagoana. Monografia de Graduação, Universidade Federal de Alagoas; 1996.

103. Lucherini M, Pessino M, Farias AA: Pampas fox Pseudalopex gymnocercus. In Canids: foxes, wolves, jackals and dogs: status survey and conservation action plan Edited by: Sillero-Zubire C, Hoffmann M, MacDonald DW. Cambridge: IUCN Canid Specialist Group; 2004

104. Lizarralde M: Ethnoecology of monkeys among the Bari of Venezuela: perception, use and conservation. In Primates Face to Face: The Conservation Implications of Human-Nonhuman Primate Interconnections Edited by: Fuentes A, Wolfe D. Cambridge: Cambridge University Press; 2002:85-100.

105. Lenko K, Papavero N: Insetos no Folclore. 2 edition. São Paulo, Brazil: Plêiade/ FAPESP; 1996

106. Jacobo-Salcedo MR, Alonso-Castro AJ, Zarate-Martinez A: Folk medicinal use of fauna in Mapimi, Durango, México. Journal of Ethnopharmacology 2010, 122(2):902-906.
107. Estrategia nacional de conservacion y uso sostenible de la biodiversidad estudio nacional de biodiversidad. [http://www.inbio.ac.cr/estrategia/ Estudio_2000/estudio/indice.html\#indigena].

108. Hernández PS: Estudio etnozoológico en la región centro-sur de la Sierra de Nanchititla. Grado Universidad Autónoma del Estado de México; 2005.

109. Gutierrez YR: Uso de Avifauna por Comunidades Tsimane' y Estudio de la Comunidad: Chirisi Beni-Bolivia. Tesis de grado Universidad Mayor de San Andrés; 2005.

110. Gonzalez-Kirchner JP, Sainz de la Maza M: Primates hunting by Guaymi amerindians in Costa Rica. Human Evolution 1998, 13:15-19.

111. Freire FC: Répteis utilizados na medicina popular no Estado de Alagoas. Monografia de Graduação, Universidade Federal de Alagoas; 1996.

112. Ferreira FS, Brito S, Ribeiro S, Saraiva A, Almeida W, Alves RRN: Animalbased folk remedies sold in public markets in Crato and Juazeiro do Norte, Ceara, Brazil. BMC Complementary and Alternative Medicine 2009, 9:17.

113. Ferreira FS, Brito S, Ribeiro S, Almeida W, Alves RRN: Zootherapeutics utilized by residents of the community Poco Dantas, Crato-CE, Brazil. Journal of Ethnobiology and Ethnomedicine 2009, 5:21.

114. Fernandes-Pinto E, Corrêa MFM: Uso medicinal da fauna silvestre pela comunidade do Tromomô, Guaraqueçaba (Paraná - Brasil). /l Simpósio Brasileiro de Etnobiologia e Etnoecologia; São Carlos 1998.

115. Costa-Neto EM: Animal-based medicines: biological prospection and the sustainable use of zootherapeutic resources. Anais da Academia Brasileira de Ciências 2005, 77:33-43.

116. Costa-Neto EM: The Use of Insects in Folk Medicine in the State of Bahia, Northeastern Brazil, With Notes on Insects Reported Elsewhere in Brazilian Folk Medicine. Human Ecology 2002, 30:245-263.

117. Costa-Neto EM: A cultura pesqueira do litoral Norte da Bahia Salvador/ Macéio: EDUFBA/EDUFAL; 2001

118. Costa-Neto EM, Oliveira MVM: Cockroach is Good for Asthma: Zootherapeutic Practices in Northeastern Brazil. Human Ecology Review 2000, 7:41-51.

119. Costa-Neto EM: Conhecimento e usos tradicionais de recursos faunísticos por uma comunidade Afro-Brasileira. Resultados preliminares. Interciencia 2000, 25:423-431.

120. Costa-Neto EM, Marques JGW: Conhecimento ictiológico tradicional e a distribuição temporal e espacial de recursos pesqueiros pelos pescadores de Conde, Estado da Bahia, Brasil. Etnoecológica 2000, 4:56-68.

121. Costa-Neto EM: Introdução a etnoentomologia: consideraçôes metodológicas e estudo de casos. 1 edition. Feira de Santana Editora Universitária da UEFS; 2000.

122. Costa-Neto EM: Zootherapy Based Medicinal Traditions in Brazil. Honey Bee 2000, 11(2):2-4

123. Bolkovic ML, Ramadori D: Manejo de Fauna Silvestre en la Argentina. Programas de uso sustentable Buenos Aires: Dirección de Fauna Silvestre, Secretaría de Ambiente y Desarrollo Sustentable; 2006.

124. Boccardo L, Costa-Neto EM, Silva TRd, Jucá-Chagas R: Insetos na medicina popular do povoado de Porto Alegre, Maracás, Bahia. In Zooterapia: Os Animais na Medicina Popular Brasileira. Volume 2. 1 edition. Edited by: CostaNeto EM, Alves RRN. Recife, PE, Brazil: NUPEEA; 2010:209-220.

125. Bernarde PS, Santos RA: Utilização medicinal da secreção ("vacina-dosapo") do anfíbio kambô (Phyllomedusa bicolor)(Anura: Hylidae) por população não-indígena em Espigão do Oeste, Rondônia, Brasil. Revista Biotemas 2009, 22:213-220.

126. Fernades-Ferreira $\mathrm{H}$ : Atividades cinegéticas em um brejo de Altitude no Nordeste do Brasil: Etnozoologia e conservação. Dissertação de Mestrado Universidade Federal da Paraíba; 2011.

127. Barajas E: Los animales usados en la medicina popular mexicana Ciudad de México: México: Imprenta Universitaria; 1961

128. Aranda M, Gual-Díaz M, Monroy-Vilchis O, Silva L, Velázquez A: Aspectos etnoecológicos: aprovechamiento de la flora y fauna silvestres en el sur de la Cuenca de México. In Biodiversidad de la región de montaña del sur de la Cuenca de México Edited by: Velázquez A, Romero F. México: UAMSecretaría del Medio Ambiente; 1999:264-283.

129. Andrade JN, Costa-Neto EM: Primeiro registro da utilização medicinal de recursos pesqueiros na cidade de São Félix, Estado da Bahia, Brasil. Acta Sci Biol Sci 2005, 27:177-183.

130. Souto WMS, Mourão JS, Barboza RRD, Rocha MSP, Alves RRN: Animal-based medicines used in ethnoveterinary practices in the semi-arid region of Northeastern Brazil. Anais da Academia Brasileira de Ciências. 
131. Alves RRN, Lima HN, Tavares MC, Souto WMS, Barboza RRD, Vasconcellos A: Animal-based remedies as complementary medicines in Santa Cruz do Capibaribe, Brazil. BMC Complementary and Alternative Medicine 2008, 8:44.

132. Confessor M, Mendonca L, Mourao J, Alves RRN: Animals to heal animals: ethnoveterinary practices in semi-arid region, Northeastern Brazil. Journal of Ethnobiology and Ethnomedicine 2009, 5:37.

133. Alves RRN, Neto NAL, Brooks SE, Albuquerque UP: Commercialization of animal-derived remedies as complementary medicine in the semi-arid region of Northeastern Brazil. Journal of Ethnopharmacology 2009, 124:600-608

134. Alves RRN: Commercialization of Uranoscodon superciliosus Linnaeus, 1758 (Tropiduridae) for magical-religious purposes in North and Northeastern of Brazil. Sitientibus Série Ciências Biológicas 2008, 8:257-258.

135. Alves RRN, Oliveira MGG, Barboza RRD, Lopez LCS: An ethnozoological survey of medicinal animals commercialized in the markets of Campina Grande, NE Brazil. Human Ecology Review 2010, 17:11-17.

136. Alves RRN, Barboza RRD, Souto WMS: A Global overview of canids used in traditional medicines. Biodiversity and Conservation 2010, 19:1513-1522.

137. Alves RRN, Mendonça LET, Confessor MVA, Vieira WLS, Lopez LCS: Hunting strategies used in the semi-arid region of northeastern Brazil. Journal of Ethnobiology and Ethnomedicine 2009, 5:12.

138. Oliveira ES, Torres DF, Brooks SE, Alves RRN: The medicinal animal markets in the metropolitan region of Natal City, Northeastern Brazil. Journal of Ethnopharmacology 2010, 130(1):54-60.

139. Alves RRN, Oliveira MGG, Barboza RRD, Singh R, Lopez LLC: Medicinal Animals as Therapeutic Alternative in a Semi-Arid Region of Northeastern Brazil. Research in Complementary Medicine 2009, 16:305-312.

140. Alves RRN, Souto WMS, Barboza RRD: Primates in traditional folk medicine: a world overview. Mammal Review 2010, 40:155-180.

141. Alves RRN, Pereira-Filho GA, Vieira KS, Santana GG, Vieira WLS, Almeida WO: Répteis e as populações humanas no Brasil: uma abordagem etnoherpetológica. In A Etnozoologia no Brasil: Importância, Status atual e Perspectivas. Volume 7. 1 edition. Edited by: Alves RRN, Souto WMS, Mourão JS. Recife, PE, Brazil: NUPEEA; 2010:121-148.

142. Alves RRN, Léo Neto NA, Santana GG, Vieira WLS, Almeida WO: Reptiles used for medicinal and magic religious purposes in Brazil. Applied Herpetology 2009, 6:257-274.

143. Alves RRN, Vieira WLS, Santana GG: Reptiles used in traditional folk medicine: conservation implications. Biodiversity and Conservation 2008, 17(8):2037-2049

144. Alves RRN, Santana GG: Use and commercialization of Podocnemis expansa (Schweiger 1812) (Testudines: Podocnemididae) for medicinal purposes in two communities in North of Brazil. Journal of Ethnobiology and Ethnomedicine 2008, 4:6.

145. Alves RRN: Use of Marine Turtles in Zootherapy in Northeast Brazil. Marine Turtle Newsletter 2006, 112:16-17.

146. Alves RRN, Rosa IL: Use of Tucuxi Dolphin Sotalia fluviatilis for Medicinal and Magic/Religious Purposes in North of Brazil. Human Ecology 2008, 36:443-447.

147. Alves RRN, Soares TC, Mourão JS: Uso de animais medicinais na comunidade de Bom Sucesso, Soledade, Paraíba. Sitientibus Série Ciências Biológicas 2008, 8:142-147.

148. Alves RRN: Uso e comércio de animais para fins medicinais e mágicoreligiosos no Norte e Nordeste do Brasil. DSc. Thesis Universidade Federal da Paraíba, Programa de Pós-Graduação em Ciências Biológicas; 2006.

149. Alves RRN, Dias TLP: Usos de invertebrados na medicina popular no Brasil e suas implicações para conservação. Tropical Conservation Science 2010, 3:159-174.

150. Souto WMS, Alves RRN, Confessor MVA, Barboza RRD, Mourão JS, Mendonça LET: A Zooterapia na Etnoveterinária do semi-árido paraibano. In A Etnozoologia no Brasil: Importância, Status atual e Perspectivas. Volume 7. 1 edition. Edited by: Alves RRN, Souto WMS, Mourão JS. Recife, PE, Brazil: NUPEEA; 2010:423-446.

151. Alves RRN, Silva CC, Barboza RRD, Souto WMS: Zootherapy as an alternative therapeutic in South America. Journal of Alternative Medicine Research 2009, 1:21-47.

152. Almeida AV, Alves AGC, Lucena RFP, Albuquerque UP: Prescrições zooterápicas indígenas brasileiras nas obras de Guilherme Piso (16111678). In Atualidades em Etnobiologia e Etnoecologia Edited by: Alves AGC, Lucena RFP, Albuquerque UP. Recife, PE, Brazil, NUPEEA; 2005:45-60.
153. Almeida AV: A zooterapia adotada pelos médicos Simão Pinheiro Morão (c. 1618-1685) e João Ferreyra da Rosa (c. 1659-1725) em Pernambuco no final do século XVII. In Zooterapia: Os Animais na Medicina Popular Brasileira. Volume 2. 1 edition. Edited by: Costa-Neto EM, Alves RRN. Recife, PE, Brazil: NUPEEA; 2010:55-74.

154. Almeida AV: Zooterapia indígena brasileira do século XVIII nas obras de Guilherme Piso, Georg Marcgrave e Joannes de Laet. Sitientibus Série Ciências Biológicas 2007, 7:261-272.

155. Alvarez M: Fortalecimiento de la Conservación de la Naturaleza Mediante las Prácticas Tradicionales de Conservación que Desarrollan las Comunidades Indígenas Cabécares de Bajo Chirripó y Nairi Awari. Fortalecimiento de la Conservación de la Naturaleza Mediante las Prácticas Tradicionales de Conservación que Desarrollan las Comunidades Indígenas Cabécares de Bajo Chirripó y Nairi Awari 2006.

156. Banks N, Lima VOA: Matérias primas animais Recife: Imprensa Universitária da UFRPE; 1986.

157. Barbarán FR: Usos mágicos, medicinales y rituales de la fauna en la Puna del Noroeste Argentino y Sur de Bolivia. Contribuciones al manejo de vida silvestre en Latinoamérica 2004, 1:01-26.

158. Begossi A, Braga F: Food taboos and folk medicine among fishermen from the Tocantins River. Amazoniana 1992, 12:341-352.

159. Begossi A: Food taboos at Búzios Island (SE Brazil): their significance and relation to folk medicine. Journal of Ethnobiology 1992, 12:117-139.

160. Begossi A, Silvano RAM, Amaral BD, Oyakama OT: Uses of Fish and Game by Inhabitants of an Extrative Reserve (Upper Juruá, Acre, Brazil). Environment, Development and Sustainability 1999, 1:73-93.

161. Branch L, Silva MF: Folk medicine in Alter do Chão, Pará, Brasil. Acto Amazonica 1983, 13:737-797.

162. Campos E: Folclore do Nordeste. 1 edition. Rio de Janeiro: Edições $O$ Cruzeiro; 1960

163. Chaves SG: Memórias de um chiquitano sobre zooterapia, município de Porto Espiridião, Mato Grosso, fronteira Brasil-Bolívia. Universidade do Estado do Mato Grosso; 2007.

164. Costa-Neto EM: Faunistc Resources used as medicines by an Afrobrazilian community from Chapada Diamantina National Park, State of Bahia-Brazil. Sitientibus 1996, 15:211-219.

165. Costa-Neto EM: Barata é um santo remédio: introdução a zooterapia popular no Estado da Bahia Feira de Santana: Editora Universitária da UEFS; 1999.

166. Costa-Neto EM: Cultura pesqueira, desenvolvimento e sustentabilidade no litoral norte do estado da Bahia: um estudo de caso. TecBahia 1999, 14:131-139.

167. Costa-Neto EM: Healing with animals in Feira de Santana City, Bahia, Brazil. Journal of Ethnopharmacology 1999, 65:225-230.

168. Costa-Neto EM: Honey bees from Brazil: diversity of insect-product used by the Pankararé. Honey Bee 1999, 10:17-18.

169. Costa-Neto EM: Recursos animais utilizados na medicina tradicional dos índios Pankararé que habitam o Nordeste do Estado da Bahia, Brasil. Actualidades Biologicas 1999, 21:69-79.

170. Marques JGW, Costa-Neto EM: Insect cure for ailments. Honey Bee 1999 10:1-17.

171. Costa-Neto EM, Dias CV, Melo MN: O conhecimento ictiológico tradicional dos pescadores da cidade de Barra, região do Médio São Francisco, Estado da Bahia, Brasil. Acta Scientiarum 2002, 24:561-572.

172. Costa-Neto EM, Marques JGW: Atividades de pesca desenvolvidas por pescadores da comunidade de Siribinha, Município de Conde, Bahia: uma abordagem Etnoecológica. Sitientibus 2001, 1:71-78.

173. Costa-Neto EM, Pacheco JM: Utilização medicinal de insetos no povoado de Pedra Branca, Santa Terezinha, Bahia, Brasil. Biotemas 2005, 18:113-133.

174. Costa MEB: Conhecimento etnoecológico e conservação de cetáceos em uma comunidade pesqueira de Canavieiras, Bahia, Brasil. Monografia de graduação Universidade Estadual de Santa Cruz; 2008.

175. Coutinho H, Vasconcellos A, Lima M, Almeida-Filho G, Alves RRN: Termite usage associated with antibiotic therapy: enhancement of aminoglycoside antibiotic activity by natural products of Nasutitermes corniger (Motschulsky 1855). BMC Complementary and Alternative Medicine 2009, 9:35.

176. De la Galvez Murillo E, Pacheco LF: Short Communication Abundancia y estructura poblacional de la lagartija jararank'o (Liolaemus signifer; Liolaemidae-Lacertilia-Reptilia) en zonas con y sin extracción comercial en el Altiplano de Bolivia. Tropical Conservation Science 2009, 2:106-115. 
177. Domus C: ElA - Prospección Sísmica 2D y Perforación Exploratoria, Lote 138 - Vol. II Cap. 2.0 SubCap 2.6-1. ElA - Prospección Sísmica 2D y Perforación Exploratoria, Lote 138 - Vol. II Cap. 2.0 SubCap 2.6-1 2010.

178. Durán EN, Cordero WJA, Betancourt SFH: Conocimiento tradicional sobre el uso y aprovechamiento de la fauna silvestre en la reserva municipal de Cuxtal, Yucatán, México. In Sistemas Biocognitivos Tradicionales: Paradigmas en la conservácion Biológica y fortalecimiento cultural Edited by: Fuentes AM, Silva MTP, Méndez RM, Azúa RV, Correa PM. Santillán TVG: Associación Etnobiológica Mexicana, A.C; 2010.

179. Figueiredo N: Os 'bichos' que curam: os animais e a medicina 'folk' em Belém do Pará. Boletim do Museu Paraense Emílio Göeldi 1994, 10:75-91.

180. Souto FJB, Andrade CTS, Souza AF: Uma abordagem etnoecológica sobre a zooterapia na medicina popular em Andaraí, Chapada Diamantina, Bahia. I Encontro Baiano de Etnobiologia e Etnoecologia; Feira de Santana, Bahia UEFS; 1999, 1-18.

181. Souto WMS: Animais de uso etnoveterinário no semi-árido paraibano: implicações para conservação e sustentabilidade. Dissertação de Mestrado Universidade Federal da Paraíba/Universidade Estadual da Paraíba, Programa de Pós-Graduação em Desenvolvimento e Meio Ambiente; 2009.

182. Tejada R, Chao E, Gómez H, Painter REL, Wallace RB: Evaluación sobre el uso de la fauna silvestre en la Tierra Comunitaria de Origen Tacana, Bolivia. Ecología en Bolivia 2006, 41:138-148.

183. Vallejo P, Aguayo R: Usos, medicinales, mágico-religiosos de los anfibios y reptiles de Bolivia. X Congreso Argentino de Herpetología; San Salvador 2009.

184. Vargas LMA: Estudio comparativo de la caza y uso de mamiferos en dos comunidades Tsimane' Provincia Ballivián - Beni. Universidad Mayor de San Andrés; 2002.

185. Venero JLG: Uso de animales en la cuenca del Vilcanota, Cusco (Perú). Estudios Atacameños 1998, 16:203-208.

186. Phyllomedusa burmeisteri. [http://es.wikipedia.org/wiki/ Phyllomedusa_burmeisteri].

187. Yahuarcani A, Morote K, Calle A, Chujandama M: Estado de conservación de Crax globulosa en la Reserva Nacional Pacaya Samiria, Loreto. Rev peru biol 2009, 15:041-049.

188. Ziemendorff S: Sustancias estimulantes y brebajes afrodisíacos en la tradición de la Amazonía peruana. Culturas populares 2008, 7:1-7.

189. Altrichter M: Wildlife in the life of local people of the semi-arid Argentine Chaco. Biodiversity and Conservation 2006, 15:2719-2736.

190. Costa-Neto EM: Folk Taxonomy and Cultural Significance of "Abeia" (Insecta, Hymenoptera) to the Pankarare, Northeastern Bahia State, Brazil. Journal of Ethnobiology 1998, 18:1-13.

191. Begossi A, Braga F: Food taboos and folk medicine among fishermen from the Tocantins River. Amazoniana 1992, 12:341-352.

192. Venero JLG: Uso de animales en la cuenca del Vilcanota, Cusco (Perú). Estudios Atacameños 1998, 16:203-208.

193. Barboza RRD, Souto WMS, Mourão JS: The use of zootherapeutics in folk veterinary medicine in the district of Cubati, Paraíba State, Brazil. Journal of Ethnobiology and Ethnomedicine 2007, 3:14.

194. ITIS (Integrated Taxonomic Information System) (2010) Catalogue of Life 2008-Annual Checklist. [http://www.catalogueoflife.org/annual-checklist/ search.php].

195. Kakati LN, Ao B, Doulo V: Indigenous Knowledge of Zootherapeutic Use of Vertebrate Origin by the Ao Tribe of Nagaland. Human Ecology 2006, 19:163-167.

196. Adeola MO: Importance of wild animals and their parts in the culture, religious festivals, and traditional medicine, of Nigeria. Environmental Conservation 1992, 19:125-134.

197. Khalid HS, El-Kamali HH, Elmanan AMA: Trade of Sudanese Natural Medicinals and their role in Human and Wildlife Health Care. Cropwatch Newsletter 2007, 1-15.

198. CITES: List of species traded for medicinal purposes. Eighteenth meeting of the animals committee. 2002

199. Souza RF: Medicina e fauna silvestre em Minas Gerais no século XVIII. Varia hist 2008, 24:273-291.

200. von Martius KFP: Natureza, doenças, medicina e remedios dos indios brasileiros (1844) Rio de Janeiro; 1939.

201. Iwu MM: Handbook of African Medicinal Plants Boca Raton (FL):CRC; 1993.

202. Descola P: The spears of twilight: life and death in the Amazon jungle Flamingo; 1996.
203. Radbill SX: Child Hygiene Among the Indians. Texas Reports on Biology and Medicine 1976, 3:419-512.

204. Janson T: Animales de Centroamérica en peligro Editorial Piedra Santa; 1981.

205. Ortiz de Montellano BR: Aztec medicine, health and nutrition New Brunswick: Rutgers University Press; 1990.

206. McCorkle CM, Martin M: Parallels and potentials in animal and human ethnomedical technique. Agriculture and Human Values 1998, 15:139-144.

207. Alves RRN, Alves HN, Barboza RRD, Souto WMS: The influence of religiosity on health. Ciência \& Saúde Coletiva 2010, 15:2105-2111.

208. Wirth DP: Implementing spiritual healing in modern medical practice: Advances. J Mind-Body Health 1993, 9:69-81.

209. Rubel AJ: The epidemiology of a folk illness: Susto in Hispanic America. In Culture, Disease, and Healing: Studies in Medical Anthropology Edited by: David L. New York: Macmillan Publishing Co; 1977:119-128.

210. Zweber A: Cultural competence in pharmacy practice. Am J Pharm Educ 2002, 66:172-176.

211. Straker G: Integrating African and Western healing practices in South Africa. American journal of psychotherapy 1994, 48:455.

212. Maher P: A review of 'traditional'Aboriginal health beliefs. Australian Journal of Rural Health 1999, 7:229-236.

213. Pieroni A, Quave CL: Traditional pharmacopoeias and medicines among Albanians and Italians in southern Italy: A comparison. Journal of Ethnopharmacology 2005, 101:258-270.

214. Stone L: Concepts of illness and curing in a central Nepal village. Contributions to Nepalese Studies 1976, 3:55-80.

215. Léo Neto NA, Brooks SE, Alves RRN: From Eshu to Obatala: animals used in sacrificial rituals at Candomble "terreiros" in Brazil. Journal of Ethnobiology and Ethnomedicine 2009, 5:1-23.

216. Léo Neto NA, Alves RRN: "Sangue e música": animais utilizados em rituais de sacrifício em terreiros de Candomblé. In A Etnozoologia no Brasil: Importância, Status atual e Perspectivas. Volume 7. 1 edition. Edited by: Alves RRN, Souto WMS, Mourão JS. Recife, PE, Brazil: NUPEEA; 2010:495-512.

217. Mata M, Campos E, Basso R, Campagnucci P, Fearnside GMJ, Magrin A, Moreno A: Latin America. In Climate Change 2001, Impacts, Adaptation, and Vulnerability A contribution of Working Group II to the Third Assessment Report of the IPCC Edited by: McCarthy JJ, Canziani O, Leary N, Dokken D, White K. Cambridge, UK and New York: Cambridge University Press; 2001.

218. Athais R: Indigenous Traditional Medicine among the Hupd'ah - Maku of Tiquie River (Brazil). 2004.

219. Sanchez G: I render services for science don't I?...and I am an indigenous descendant. 2004.

220. Tabuti JRS, Dhillion SS, Lye KA: Ethnoveterinary medicines for cattle (Bos indicus) in Bulamogi county, Uganda: plant species and mode of use. Journal of Ethnopharmacology 2003, 88:279-286.

221. Sofowora A: Medicinal Plants and Traditional Medicine in Africa. 2 edition. Ibadan: Spectrum Books Ltda; 1993.

222. Luoga EJ, Witkowski ETF, Balkwill K: Differential utilization and ethnobotany of trees in Kitulanghalo forest reserve and surrounding communal lands, eastern Tanzania. Econ Bot 2000, 54:328-343.

223. De Smet PAGM: Is there any danger in using traditional remedies? J Ethnopharmacol 1991, 32:43-50.

224. Still J: Use of animal products in traditional Chinese medicine: environmental impact and health hazards. Complementary Therapies in Medicine 2003, 11:118-122.

225. Astudillo VM, Zottele AC, Dora F: Livestock development and animal health in Latin America. Bol Centr Panam Fiebre Aftosa 1991, 57:15-22.

226. Schnurrenberger PR, Hubbert WT: An outline of the zoonoses Ames, IA: lowa State University Press; 1981.

227. Pujol FH: Virus en primates no humanos: Zoonosis, Antroponosis y Biodiversidad. Interciencia 2006, 31:396-402.

228. Swift G: Park culls colony of diseased monkeys London: International Express; 2000.

229. Goudsmit J: Viral sex-The nature of AIDS New York: Oxford University Press; 1997.

230. Pieroni A, Quave C, Nebel S, Heinrich M: Ethnopharmacy of the ethnic Albanians (Arbereshe) of northern Basilicata, Italy. Fitoterapia 2002, 73:217-241.

231. Ferreira FS, Brito SV, Costa JGM, Alves RRN, Coutinho HDM, Almeida WdO: Is the body fat of the lizard Tupinambis merianae effective against bacterial infections? Journal of Ethnopharmacology 2009, 126:233-237. 
232. Paavilainen HM: Medieval pharmacotherapy, continuity and change: case studies from Ibn Sina and some of his late Medieval commentators Boston: Brill Academic Pub; 2009

233. Trowell S: Drugs from Bugs: The Promise Of Pharmaceutical Entomology. Futurist 2003, 37:17-19.

234. Dossey AT: Insects and their chemical weaponry: New potential for drug discovery. Natural Product Reports 2010, 27:1737-1757.

235. Chivian E: Biodiversity: Its importance to human health Boston: Harvard Medical School; 2002.

236. Costa-Neto EM: Sustainable development and traditional knowledge: a case study in a Brazilian artisanal fishermen's community. Sustainable Development 2000, 8:89-95.

237. Monteiro JM, Araújo EL, Amorim ELC, Albuquerque UP: Local Markets and Medicinal Plant Commerce: A Review with Emphasis on Brazil. Economic Botany 2010, 64:352-366.

238. Bolze D, Chetkiewicz C, Qiu M, Krakower D: The Availability of Tiger-based Traditional Chinese Medicine Products and Public Awareness about the Threats to the Tiger in New York's Chinese Communities: a pilot study. Wildlife Conservation Society Working Paper; 1998.

239. Servheen C: The trade in bears and bear parts. In Bears: status survey and conservation action plan Edited by: Servheen C, Herrero S, Peyton B 1999, 33-38.

240. Callister DJ, Bythewood T: Of Tiger Treatments \& Rhino Remedies: Trade in Endangered Species Medicines in Australia and New Zealand Traffic Oceania; 1995.

241. Marshall NT: Searching for a cure: conservation of medicinal wildlife resources in East and Southern Africa. Traffic International; 1998

242. Kritsky G: Insects in traditional Chinese medicines. Proceedings of the Indiana Academy of Science 1987, 96:289-291.

243. Joseph ANT: The relevance of traditional bird drugs in relation to modern primary healthcare in Madhya Pradesh. Records of the Zoological Survey of India 1990, 86:193-210.

244. Celso R: Criação de condições e incentivos para a conservação local de biodiversidade. In A estratégia global da biodiversidade Diretrizes de ação para estudar, salvar e usar de maneira sustentável e justa a riqueza biótica da Terra Edited by: Speth JC, Holdgate MW, Tolba MK. Rio de Janeiro: WRI/ UICN/PNUMA; 1992:92-93.

245. Soejarto DD: Biodiversity prospecting and benefit-sharing: perspectives from the field. Journal of Ethnopharmacology 1996, 51:1-15.

246. Anyinam C: Ecology and ethnomedicine: Exploring links between current environmental crisis and indigenous medical practices. Social Science \& Medicine 1995, 40:321-329.

247. McMichael AJ, Beaglehole R: The changing global context of public health. The Lancet 2000, 356:495-499.

248. Alves RRN, Rosa IL: Medicinal animals for the treatment of asthma in Brazil. BMC Complementary and Alternative Medicine 2008, 14:350-351.

249. Alves RRN, Souto WMS: Etnozoologia: conceitos, considerações históricas e importância. In A Etnozoologia no Brasil: Importância, Status atual e Perspectivas. Volume 7. 1 edition. Edited by: Alves RRN, Souto WMS, Mourão JS. Recife, PE, Brazil: NUPEEA; 2010:19-40.

doi:10.1186/1746-4269-7-9

Cite this article as: Alves and Alves: The faunal drugstore: Animal-based remedies used in traditional medicines in Latin America. Journal of Ethnobiology and Ethnomedicine 2011 7:9.

\section{Submit your next manuscript to BioMed Central and take full advantage of:}

- Convenient online submission

- Thorough peer review

- No space constraints or color figure charges

- Immediate publication on acceptance

- Inclusion in PubMed, CAS, Scopus and Google Scholar

- Research which is freely available for redistribution

Submit your manuscript at www.biomedcentral.com/submit 